\title{
A Comprehensive Approach for \\ Bulk Power System Reliability Assessment
}

\author{
A Thesis \\ Presented to \\ The Academic Faculty
}

by

\section{Fang Yang}

\author{
In Partial Fulfillment \\ of the Requirements for the Degree \\ Doctor of Philosophy in the \\ School of Electrical and Computer Engineering
}

Georgia Institute of Technology

May 2007 


\section{A Comprehensive Approach for Bulk Power System Reliability Assessment}

Approved by:

Professor A. P. Sakis Meliopoulos Professor Ronald G. Harley School of Electrical and Computer School of Electrical and Computer Engineering Engineering

Georgia Institute of Technology Georgia Institute of Technology

Professor Deepak M. Divan

Professor Erik I. Verriest

School of Electrical and Computer Engineering School of Electrical and Computer Georgia Institute of Technology Engineering Georgia Institute of Technology

Professor Shijie Deng

School of Industrial and Systems Engineering Georgia Institute of Technology 
To my parents

Xufei Yang and Shuying Li

and to my husband

Zhao Li 


\section{ACKNOWLEDGEMENTS}

I am very grateful to all the people who have supported me during the pursuit of my

Ph.D. degree. This work would not have been possible without their encouragement and help.

First and foremost, I would like to express my sincere appreciation and deep gratitude to my dissertation advisor, Dr. A. P. Meliopoulos, for his continuous support and invaluable guidance during my graduate studies at Georgia Tech. His willingness to share freely his vast experience and knowledge has contributed significantly to the completion of this work.

I would like to extend my appreciation to Dr. Deepak M. Divan, Dr. Ronald G. Harley, and Dr. Erik I. Verriest for serving on my dissertation proposal committee, and Dr. Shijie Deng for serving on my dissertation defense committee. Their valuable comments and instructive suggestions have helped improve the quality of this dissertation.

I would also like to thank scholars and colleagues working with the Electric Power System Lab at Georgia Tech. First among these is Dr. George J. Cokkinides, I am thankful for his constant help with my research. Special thanks go to George Stepfopoulos, Quang Dam Binh, and Ramiz Alaileh. Their friendship and assistance made my stay in this lab an enjoyable experience.

I am deeply indebted to my parents and sister for their patience, support, and love. I am very grateful to my husband, Zhao Li, whose understanding, encouragement, and love have been the source of my courage to face challenges. 


\section{TABLE OF CONTENTS}

ACKNOWLEDGEMENTS ............................................................. IV

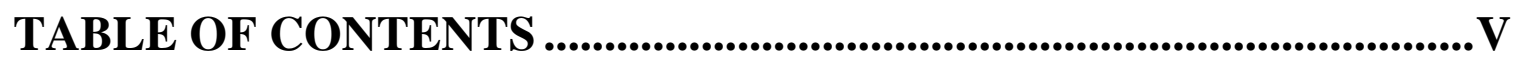

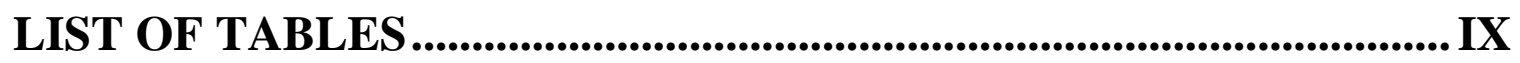

LIST OF FIGURES ..........................................................................

ABBREVIATIONS....................................................................................XIII

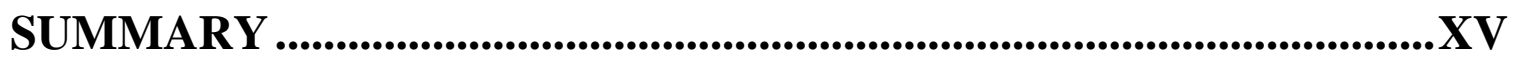

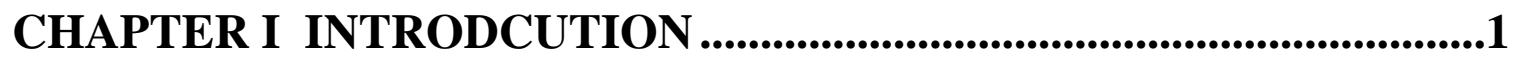

1.1 BULK POWER SYSTEM RELIABILITY ....................................................... 1

1.2 POWER INDUSTRY RESTRUCTURING ........................................................ 3

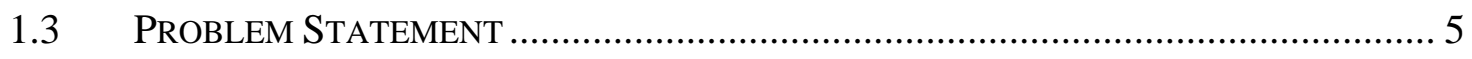

1.3.1 Large Size of System State Space ........................................................ 6

1.3.2 Effects Analysis of Critical Contingencies ............................................. 9

1.3.3 The Effects of Protection System Hidden Failures on Bulk Power System

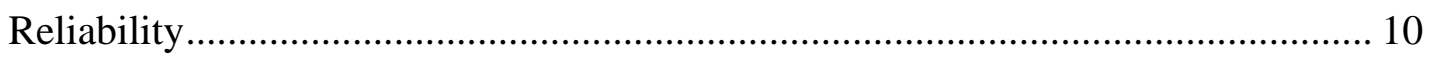

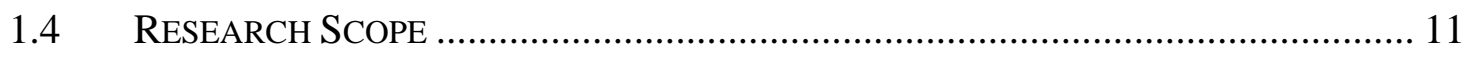

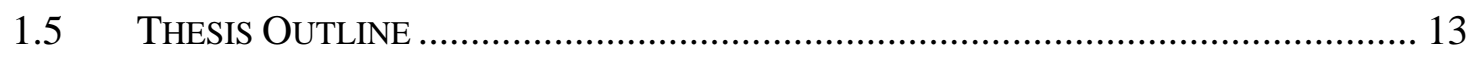

CHAPTER II OVERVIEW OF BULK POWER SYSTEM

RELIABILITY ASSESSMENT ...............................................................15

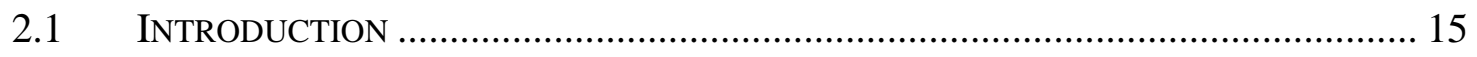


2.2 Bulk Power System Reliability Modeling Methods .............................. 15

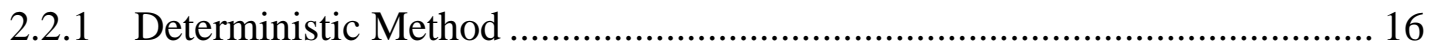

2.2.2 Probabilistic Method............................................................................. 17

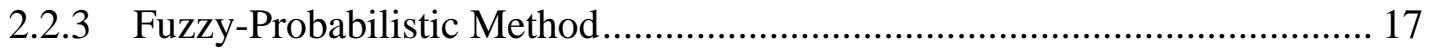

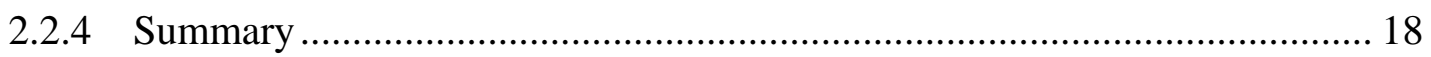

2.3 Bulk Power System Reliability Assessment Methods........................... 18

2.3.1 Analytical and Monte Carlo Simulation Techniques.................................. 19

2.3.2 The Comparison of Two Techniques...................................................... 20

2.4 ADEQUACY AND SECURITY RELIABILITY ASSESSMENT ................................. 21

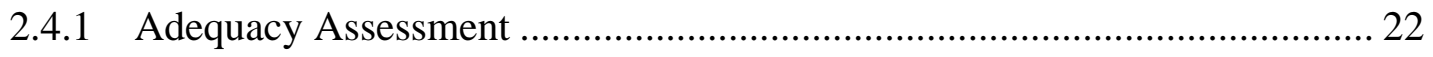

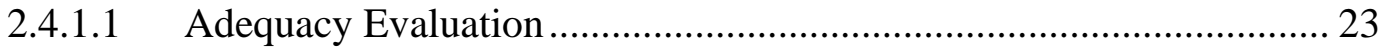

2.4.1.2 Security-Constrained Adequacy Evaluation (SCAE) ....................... 27

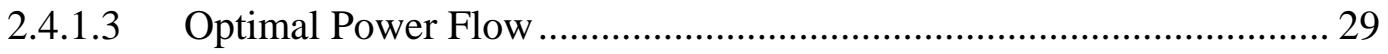

2.4.2 Integrated Adequacy and Security Assessment ....................................... 31

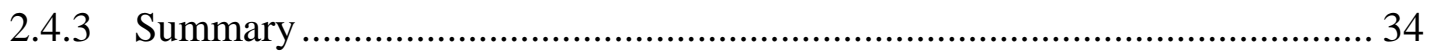

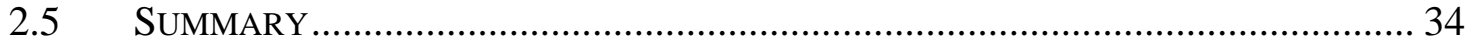

\section{CHAPTER III BULK POWER SYSTEM RELIABILITY}

ASSESSMENT METHODOLOGY .........................................................36

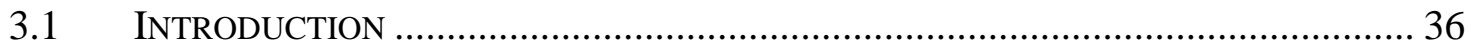

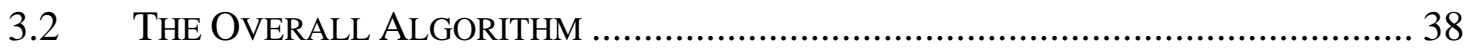

3.3 The Single-Phase Quadratized Power Flow Model ............................. 41

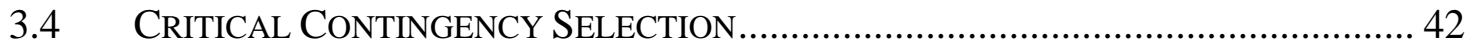

3.4.1 The Performance Index Linearization Approach...................................... 43 
3.4.2 The System State Linearization Approach ...................................................... 46

3.4.3 The Wind-Chime Contingency Enumeration Scheme.................................... 49

3.5 The ConTINGENCY EFFECTS ANALYSIS ......................................................... 50

3.5.1 Remedial Actions ..................................................................................... 51

3.5.2 The Non-Divergent Optimal Quadratized Power Flow Algorithm .............. 54

3.6 The Application of THE NDOQPF Algorithm In DeRegulated POWER

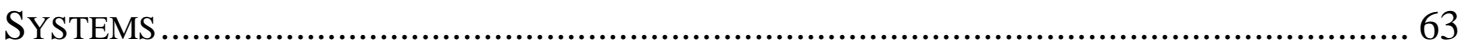

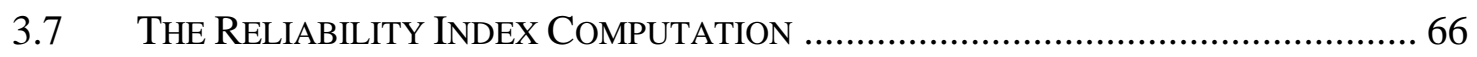

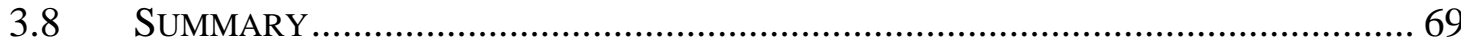

\section{CHAPTER IV EFFECTS OF PROTECTION SYSTEM HIDDEN}

FAILURES ON BULK POWER SYSTEM RELIABILITY ..................70

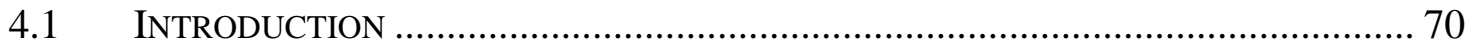

4.2 PRoteCtION SYSTEM HIDDEN FAILURES......................................................... 71

4.3 The BREaKeR-ORIEnTEd SubStation Model …......................................... 73

4.4 The IMPaCt OF AdVANCED System Monitoring AND ANALysis

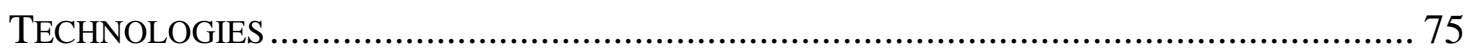

4.5 Probabilistic Modeling OF Hidden FaILURes in the Circuit BreaKer Trip

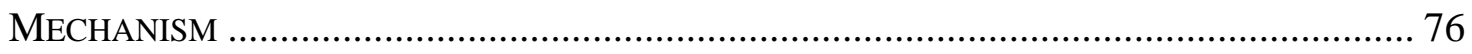

\section{CHAPTER V APPROACH DEMONSTRATION AND}

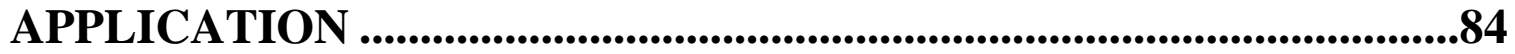

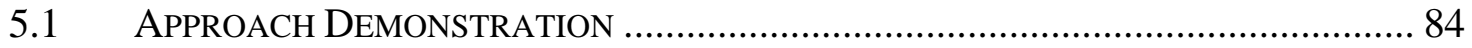

5.1.1 System State Linearization Approach............................................................ 86

5.1.2 Non-Divergent Optimal Quadratized Power Flow Algorithm....................... 90 
5.1.3 Protection System Hidden Failure Effects Analysis.....

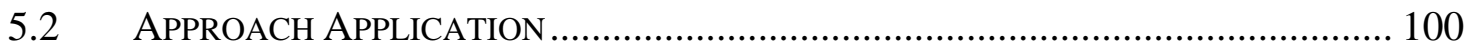

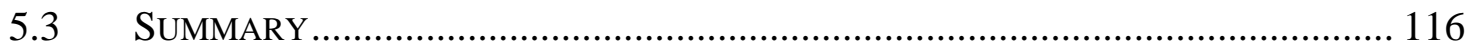

CHAPTER VI CONCLUSIONS AND RECOMMENDATIONS ...... 117

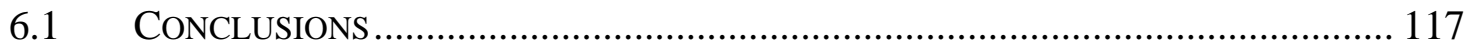

6.2 RECOMMENDATIONS FOR FUTURE WORK................................................ 119

REFERENCES ........................................................................................... 121 


\section{LIST OF TABLES}

Table 3.1: List of typical power system remedial actions. ........................................ 52

Table 4.1: State enumeration for the example substation (states 1-16)......................... 79

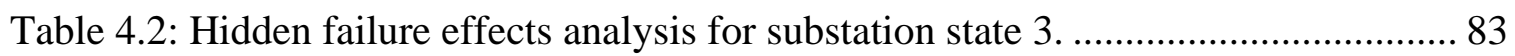

Table 5.1: Changes in the current-based circuit loading index and contingency ranking results for independent transmission circuit outages. ................................... 88

Table 5.2: Changes in the voltage index and contingency ranking results for independent

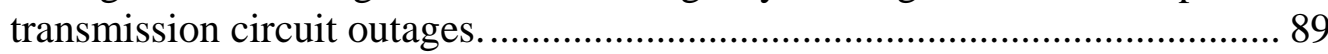

Table 5.3: Changes in the current-based circuit loading index and contingency ranking results for independent generating unit outages........................................ 90

Table 5.4: Part of the effects analysis results of first-level contingencies....................... 91

Table 5.5: Substation topology summary of the circuit breaker-oriented 24-substation

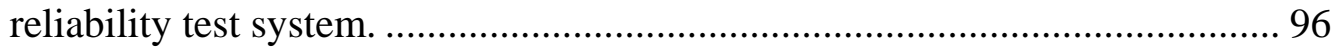

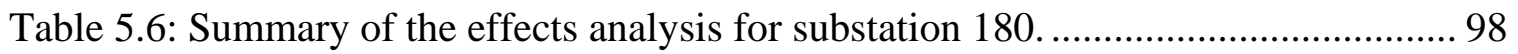

Table 5.7: List of contingencies leading to system unreliability.................................. 99

Table 5.8: Comparison of reliability indices with and without contingencies resulting from hidden failure outages.

Table 5.9: The substation topology summary of the circuit breaker-oriented 73susbstation reliability test system....

Table 5.10: Enumeration of outage event combinations given the transmission circuit and generating unit outage levels as two and one, respectively....................... 102

Table 5.11: The number of discrete values of independent load level control variables and corresponding load levels 104

Table 5.12: The reliability assessment of the 24-substation reliability test system (circuit outage level: 1 , generating unit outage level: 0 ). 107

Table 5.13: The reliability assessment of the 24-substation reliability test system (circuit outage level: 2, generating unit outage level: 0 ). 
Table 5.14: The reliability assessment of the 24-substation reliability test system........ 110

Table 5.15: The reliability assessment of the 24-substation reliability test system (circuit

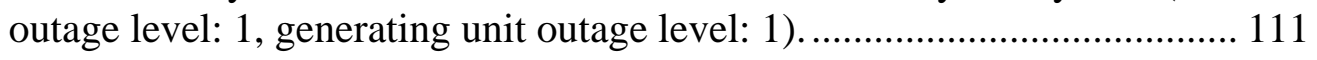

Table 5.16: The reliability assessment of the circuit breaker-oriented 73-substation reliability test system (circuit outage level: 1, generating unit outage level: 0 ).

Table 5.17: The reliability assessment of the 73-Substation reliability test system (circuit

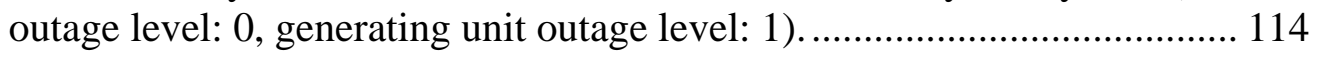

Table 5.18: Three different load levels used in the two systems ................................. 115 


\section{LIST OF FIGURES}

Figure 1.1: Hierarchical levels for power system reliability assessment......................... 2

Figure 1.2: A two-state Markov component model..................................................... 7

Figure 1.3: A generating unit model with one derated state......................................... 7

Figure 1.4: A common-mode failure model with two components................................ 8

Figure 3.1: Overall computational algorithm of the SCAE methodology....................... 38

Figure 3.2: Plot of the circuit-loading index vs. the contingency control variable $\mathrm{u}_{\mathrm{c} .} \ldots . . .48$

Figure 3.3: Plot of the voltage index vs. the contingency control variable $\mathrm{u}_{\mathrm{c} .} \ldots \ldots \ldots \ldots \ldots . . . . . . .48$

Figure 3.4: Wind-chime contingency enumeration scheme ......................................... 49

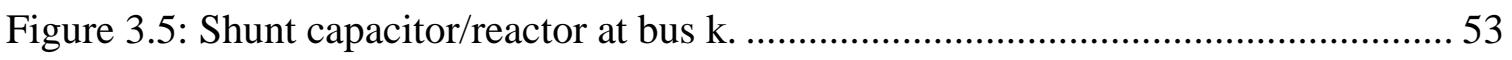

Figure 3.6: A general bus $k$ in a power system with a fictitious current source.............. 55

Figure 3.7: Flow chart of the non-divergent optimal quadratized power flow algorithm. 62

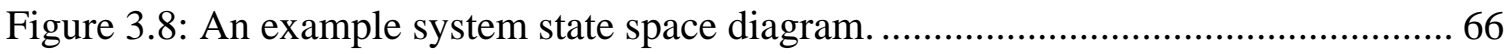

Figure 4.1: A breaker-and-a-half bus arrangement substation model............................ 74

Figure 4.2: Two-state Markov models of CBTMs. ..................................................... 77

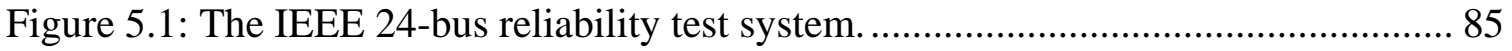

Figure 5.2: A circuit breaker-oriented, 24-substation reliability test system................... 94

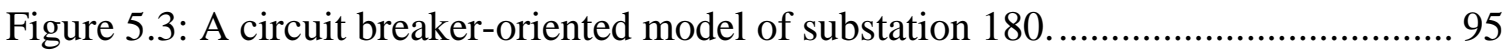

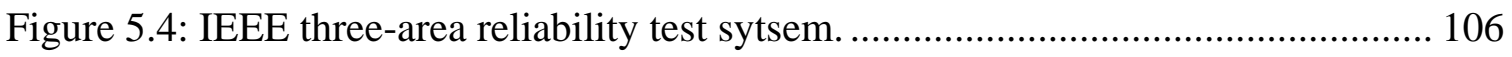

Figure 5.5: The probability indices of the 24-substation reliability test system under different component outage modes and load levels .................................. 107

Figure 5.6: The frequency indices of the 24-substation reliability test system under different component outage modes and load levels ................................. 108 
Figure 5.7: The probability indices of the 24-substation reliability test system under different component outage modes and load levels

Figure 5.8: Frequency indices of the 24-substation reliability test system under different component outage modes and load levels.

Figure 5.9: The probability indices of the 24-substation reliability test system under different load levels (circuit outage level: 0, generating unit outage levels: 1 and 2).

Figure 5.10: The frequency indices of the 24-substation reliability test system under different load levels (circuit outage level: 0, generating unit outage levels: 1 and 2).

Figure 5.11: The probability contribution from both circuit outages and generating unit outages under the independent outage mode and the low load level for 24substation reliability test system.

Figure 5.12: The comparison of probability indices of the 24-substation system and the 73-substation system under different component outage modes and the peak load level.....

Figure 5.13: The comparison of frequency indices of the 24-substation system and the 73substation system under different component outage modes and the peak load level

Figure 5.14: The comparison of probability indices of the 24-substation system and the 73-substation system under different load levels and independent component outage.

Figure 5.15: The comparison of frequency indices of the 24-substation system and the 73substation system under different load levels and independent component outage mode (circuit outage level: 0 , generating unit outage level: 1)..... 115 


\section{ABBREVIATIONS}

\begin{tabular}{|c|c|}
\hline AI & Area of Interest \\
\hline CA ISO & California Independent Transmission Operator \\
\hline $\mathrm{CB}$ & Circuit Breaker \\
\hline CBTM & Circuit Breaker Trip Mechanism \\
\hline CT & Current Transformer \\
\hline DCS & Design Contingency Set \\
\hline ERCOT ISO & Electric Reliability Council of Texas Independent Transmission Operator \\
\hline FERC & Federal Energy Regulatory Commission \\
\hline $\mathrm{HF}$ & Hidden Failure \\
\hline HL & Hierarchical Level \\
\hline IA & Interconnected Area \\
\hline IED & Intelligent Electronic Device \\
\hline ISO & Independent Transmission Operator \\
\hline LP & Linear Programming \\
\hline MISO & Midwest Independent Transmission Operator \\
\hline NDOQPF & Non-Divergent Optimal Quadratized Power Flow \\
\hline NY ISO & New York Independent Transmission Operator \\
\hline OPF & Optimal Power Flow \\
\hline PI & Performance Index \\
\hline PJM RTO & Pennsylvania-New Jersey-Maryland Regional Transmission Organization \\
\hline PMU & Phasor Measurement Unit \\
\hline
\end{tabular}




$\begin{array}{ll}\text { RA } & \text { Remedial Action } \\ \text { RTO } & \text { Regional Transmission Organization } \\ \text { RTU } & \text { Remote Terminal Unit } \\ \text { SCAE } & \text { Security-Constrained Adequacy Evaluation } \\ \text { SPQPF } & \text { Single-Phase Quadratized Power Flow } \\ \text { TC } & \text { Trip Coil } \\ \text { TPF } & \text { Traditional Power Flow } \\ \text { VT } & \text { Voltage Transformer }\end{array}$




\section{SUMMARY}

The goal of this research is to advance the state of the art in bulk power system reliability assessment. Bulk power system reliability assessment is an important procedure at both power system planning and operating stages to assure reliable and acceptable electricity service to customers. With the increase in the complexity of modern power systems and advances in the power industry toward restructuring, the system models and algorithms of traditional reliability assessment techniques are becoming obsolete as they suffer from nonrealistic system models and slow convergence (even non-convergence) when multi-level contingencies are considered and the system is overstressed. To allow more rigor in system modeling and higher computational efficiency in reliability evaluation procedures, this research proposes an analyticallybased security-constrained adequacy evaluation (SCAE) methodology that performs bulk power system reliability assessment.

The SCAE methodology adopts a single-phase quadratized power flow (SPQPF) model as a basis and encompasses three main steps: (1) critical contingency selection, (2) effects analysis, and (3) reliability index computations. In the critical contingency selection, an improved contingency selection method is developed using a wind-chime contingency enumeration scheme and a performance index approach based on the system state linearization technique, which can rank critical contingencies with high accuracy and efficiency. In the effects analysis for selected critical contingencies, a non-divergent optimal quadratized power flow (NDOQPF) algorithm is developed to (1) incorporate major system operating practices, security constraints, and remedial actions in a 
constrained optimization problem and (2) guarantee convergence and provide a solution under all conditions. This algorithm is also capable of efficiently solving the ISO/RTO operational mode in deregulated power systems. Based on the results of the effects analysis, reliability indices that provide a quantitative indication of the system reliability level are computed. In addition, this research extends the proposed SCAE framework to include the effects of protection system hidden failures on bulk power system reliability.

The overall SCAE methodology is implemented and applied to IEEE reliability test systems, and evaluation results demonstrate the expected features of proposed advanced techniques. Finally, the contributions of this research are summarized and recommendations for future research are proposed. 


\section{CHAPTER I \\ INTRODUCTION}

\subsection{Bulk Power System Reliability}

An electric power system is generally composed of three parts: (1) generation, (2) transmission, and (3) distribution systems, all of which contribute to the production and transportation of electric energy to customers. The reliability of an electric power system is defined as the probability that the power system will perform the function of delivering electric energy to customers on a continuous basis and with acceptable service quality [1]. For the purpose of conducting power system reliability assessment, the three power system parts are combined into different system hierarchical levels, as shown in Figure $1.1[2,3]$. Hierarchical level 1 (HL1) involves the reliability analysis of only the generation system, hierarchical level 2 (HL2) includes the reliability evaluation of the composite of both generation and transmission systems, referred to as the bulk power system or the composite power system, and hierarchical level 3 (HL3) consists of a reliability study of the entire power system.

At the present stage of development, the reliability evaluation of the entire power system (HL3) is usually not conducted because of the immensity and complexity of the problem in a practical system. Instead, power system reliability is assessed separately for the generation system (HL1), the bulk power system (HL2), and the distribution system [4]. Reliability analysis methods for generation and distribution systems are well 
developed. However, reliability assessment techniques for bulk power systems are not as well developed due to difficulties arising from the huge computational burden associated with the bulk power system reliability analysis [1]. Thus, this research concentrates on the area of bulk power system reliability assessment.

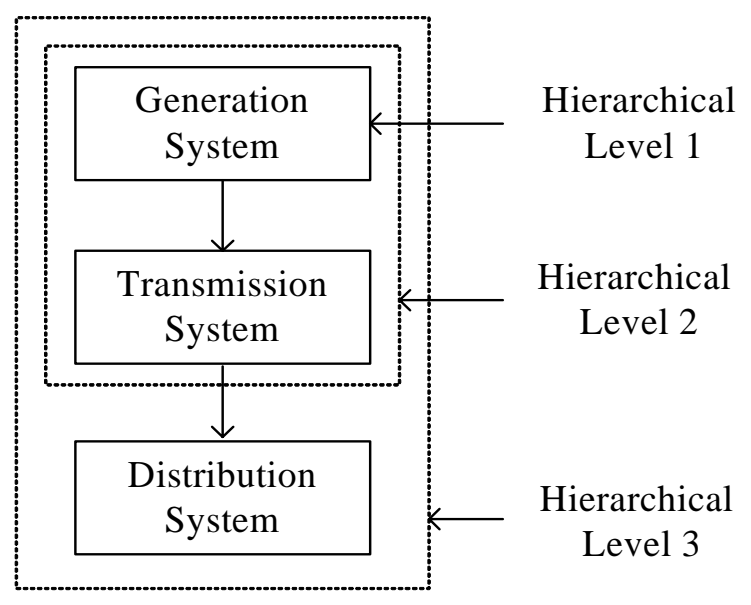

Figure 1.1: Hierarchical levels for power system reliability assessment.

Bulk power system reliability assessment refers to the process of estimating the ability of the system to simultaneously (a) generate and (b) move energy to load supply points [4]. Traditionally, it has formed an important element of both power system planning and operating procedures.

The main objective of power system planning is to achieve the least costly design with acceptable system reliability. For this purpose, long-term reliability evaluation is usually executed to assist long-range system planning in the following aspects: (1) the determination of whether the system has sufficient capacity to meet system load demands, (2) the development of a suitable transmission network to transfer generated energy to customer load points, (3) a comparative evaluation of expansion plans, and (4) a review of maintenance schedules $[5,6]$. 
Power system operating conditions are subject to changes such as load uncertainty, i.e., the load may be different from that assumed in design studies, and unplanned component outages. To deliver electricity with acceptable quality to customers at minimum cost and to prevent cascading sequences after possible disturbances, short-term reliability prediction that assists operators in day-to-day operating decisions is needed. These decisions include determining short-term operating reserves and maintenance schedules, adding additional control aids and short lead-time equipment, and utilizing special protection systems [7, 8].

\subsection{Power Industry Restructuring}

The traditional electric power industry was characterized by the monopolistic but regulated market mechanism and the bundled utility structure. For example, in the United States, a large number of electric utilities controlled electric service to customers in different control areas before the Federal Energy Regulatory Commission (FERC) issued

Orders 888 and 889, which encouraged wholesale competition in 1996 [9]. As a result, a utility in one control area became a regional monopoly, i.e., customers in this area could not select their power source and had to buy energy from the local utility. On the other hand, each electric utility was subjected to governmental regulation. For example, any request for an electric rate hike had to be approved by the local regulatory commission. Inside each control area, the electric utility was vertically bundled, supplying a series of electric services to customers, including generation, transmission, distribution, and all 
operational and maintenance activities required to keep the system operating under normal conditions [10].

In recent years, many countries have deregulated their electric power industries at various levels. The conventional vertically-integrated power utility structure, consisting of generation, transmission, and distribution segments, was unbundled to functionally separate entities. In restructured power systems, generation and distribution services are provided by independent generation and distribution companies, respectively, while electricity transmission systems with open access are overseen by independent transmission operators (ISOs) or regional transmission organizations (RTOs) [11]. The purpose of power system restructuring is to change the monopolistic but regulated power industry into a competitive power market under a deregulated environment in which power market participants (power suppliers and consumers) are allowed to buy and sell power based on their economic profits. The ultimate goal of the competition mechanism in power markets is to decrease electricity prices for consumers.

Power industry deregulation has resulted in notable changes in the system operating condition, compared to that in the traditional regulated power system [12-14]. First, the transmission system that transfers electric energy over long distances is more heavily used in the deregulated environment. The reason for the increased use is merely economic, i.e., large wholesale energy buyers are able to satisfy their needs by purchasing less expensive energy from geographically distant regions, which tends to overload the transmission system. Second, power system operating conditions may vary over a much wider range in the deregulated environment. Transmission open access and non-utility generation have prompted the more competitive environment, making it 
possible for customers to choose their suppliers. As a result, the operating condition of the system may vary considerably according to the customer demand and choice, and some could be dramatically different from what they were before or what they are forecasted to be. In addition, since power consumers have more freedom to choose lowercost energy providers from the power producers in the deregulated environment, the operating conditions of a system can change more frequently.

These scenarios triggered by power system restructuring indicate that deregulated power systems are more often operated in patterns that were not previously observed, which inevitably force system operating points very close to their physical limits $[15,16]$. Consequently, new concerns about power system reliability assessment have risen. Specifically, advanced reliability assessment techniques that allow more rigor in system modeling and higher computational efficiency in the reliability evaluation procedure are required to facilitate power market effectiveness while maintaining an acceptable system reliability level.

\subsection{Problem Statement}

Bulk power system reliability assessment involves two major challenges: (1) the large size of the state space of a practical system and (2) the need to realistically and efficiently perform the effects analysis of critical contingencies [4]. In the area of the effects analysis, since hidden failures in the protection system [17] have recently been identified as a major contributing factor to power system unreliability [128-130], the evaluation of 
the effects of hidden failures in the protection system has become another major concern in bulk power system reliability assessment.

\subsubsection{Large Size of System State Space}

One of the major impediments in bulk power system reliability assessment is the large size of the practical system state space. The entire system state space contains all possible system states (contingencies). Each system state is defined as a particular condition in which every component is in a given operating state of its own. The operating states that components may experience are determined by (a) component operating characteristics and (b) component outage modes, which are described in detail as follows.

Component Operating Characteristics Since a bulk power system is composed of generation and transmission systems, components involved in the bulk power system reliability study are mainly generating units and transmission circuits (transmission lines or transformers). The operating state of a transmission circuit is either working or failed. Such an operating characteristic of a circuit can be modeled with a two-state (up or down) Markov model, as shown in Figure 1.2. However, the operating characteristic of a generating unit is not as simple as the two-state up/down procedure. Generating units are a type of complex equipment with many auxiliary devices, such as pulverizers, water pumps, fans, and so on. Some failures of auxiliary devices may cause a generating unit to experience a number of partial failure states in which a generating unit continues to operate, but it does so at reduced capacity. Such states are known as derated states of the 
generating unit $[2,4]$. The simplest generating unit model accounting for one derated state in addition to the fully capacity and completely failed states is shown in Figure 1.3.

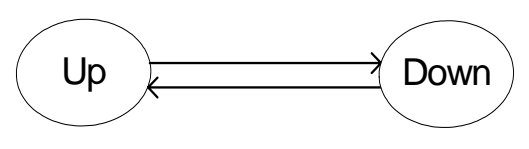

Figure 1.2: A two-state Markov component model.

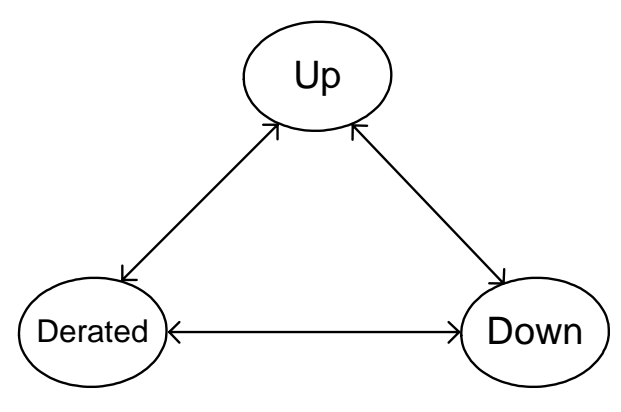

Figure 1.3: A generating unit model with one derated state.

Component Outage Modes Component outage modes generally considered in bulk power system reliability assessment include independent and common-mode outages. Independent outages refer to component failures resulting from unrelated causes. Each component involved in independent outages can be represented with a model as shown in Figure 1.2 or 1.3. The operating state of each component is independent to the operating states of other components. Common-mode outages refer to the simultaneous failures of several components resulting from a single cause [4]. One example of a common-mode failure is the failure of transmission lines on the same right-of-way that are hit by lightning. Note that the components involved in a common-mode outage can still fail independently without influencing the failure of other components. When the commonmode outage is concerned, the components involved in a common-mode outage are considered as a single entity. A common-mode outage model of such an entity that 
involves two components is illustrated in Figure 1.4, which contains four operating states and possible transitions among them. Note that such a model represents both independent and common-mode outages that the two components can experience.

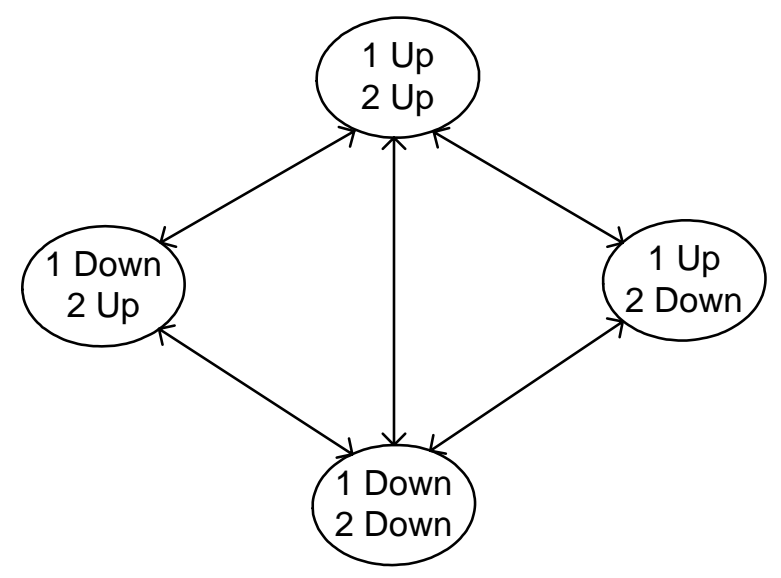

Figure 1.4: A common-mode failure model with two components.

The analysis of the operating states that system components may reside in and transit to found that in a large-scale bulk power system, including a large amount of generating units and transmission circuits, the number of total system states in the system state space is enormous. Specifically, for a system with $n$ components, if only independent component outages occur, and each component model is simplified to contain only two operating states (up or down), the entire system state space includes a total of $2^{n}$ system states. When $n$ is 2000 , the number of system states in the state space is more than $10^{600}$. If all possible system states (contingencies) are analyzed one by one for the purpose of identifying the critical contingencies that contribute to system unreliability, the contingency analysis procedure requires too much computational effort and becomes impractical. Therefore, effective state space reduction techniques that can navigate to 
identify critical contingencies rather than evaluating every contingency in the huge state space should be developed.

\subsubsection{Effects Analysis of Critical Contingencies}

The most important but computationally demanding procedure in bulk power system reliability assessment is the effects analysis of critical contingencies [4], which evaluates the impact of component failures on system operation and classifies system contingencies into successful and failed categories according to pre-defined system reliability criteria. The system reliability criteria selected in advance define the events or conditions that indicate system unreliability, such as the loss of system load or the violation of certain operating constraints. Any contingencies that the effects analysis identifies as leading to system unreliability fall into the failed category. Other evaluated contingencies fall into the successful category. The results for both categories of contingencies are used to calculate reliability indices, which quantitatively measure the level of system reliability.

With the aim of realistically evaluating component failure effects and how the system operating condition can be affected, the contingency effects analysis requires the consideration of practical system responses, such as remedial actions, protection system hidden failures, possible system transient behaviors, and so on, in addition to the traditional power flow analysis. However, most current effects analysis techniques conduct only traditional power flow analysis to obtain the system post-contingency situation, which lacks the capability of realistically analyzing whether a contingency leads to the unreliability of a system. 
Moreover, since the effects analysis must not only be performed for every contingency that is examined in the reliability evaluation but may also have to be repeated more than once for each contingency if multiple load levels are considered, the efficiency of such analysis techniques is also critical to the overall performance of the bulk power system reliability assessment. Traditional power flow analysis, however, often suffers from slow convergence (even non-convergence) when the system is severely stressed by contingencies. As a result, conducting the effects analysis in a realistic and efficient manner is another major challenge existing in bulk power system reliability assessment.

\subsubsection{The Effects of Protection System Hidden Failures on Bulk Power System Reliability}

A hidden failure in the protection system is defined in [17] as "a permanent defect that will cause a relay or a relay system to incorrectly and inappropriately remove a circuit element(s) as a direct consequence of another switching event.” In other words, hidden failures may cause multiple component outages that are dependent upon each other, i.e., an initial component outage can lead to the cascading tripping of other components because of a malfunction in the protection system. In addition, such hidden failures remain hidden in normal system conditions, and in the event of a system disturbance such as a fault or an overload, hidden failures are exposed and cause unnecessary outages, which stresses the system even more and reduces the system reliability level. Thus, hidden failures in the protection system have been identified as the major contributing factor to power system unreliability [128-130]. 
In the current practice of bulk power system reliability assessment, however, protection systems are generally assumed to be perfect, and hidden failures in protection systems are not taken into account. Specifically, system substations are represented with simplified buses, and different transmission lines simply converge at the buses to connect generators or to serve loads. The substation configuration and the associated protection system scheme are ignored in the present bulk power system network model. Therefore, a more detailed bulk power system model that includes information about substations and protection systems should be applied so that the effects of protection system hidden failures on bulk power system reliability can be considered, and appropriate techniques should be developed to identify any possible hidden failure outages.

\subsection{Research Scope}

This research aims to advance the state of the art in bulk power system reliability assessment. In particular, a comprehensive approach for bulk power system reliability assessment, i.e., the security-constrained adequacy evaluation (SCAE) methodology, is developed based on analytical techniques. In this methodology, the probabilistic model is applied to represent practical system behavior, and the following areas are investigated to address major challenges encountered in the area of bulk power system reliability assessment.

Critical Contingency Selection Since the complete contingency evaluation of the entire state space is impractical for large-scale bulk power systems, a variety of critical 
contingency selection techniques have been developed for the purpose of conducting a rapid preliminary identification of critical contingencies in the state space. In this research, based on the traditional performance index (PI) approach, the quadratized power flow model, and the wind-chime contingency enumeration scheme, an improved critical contingency selection technique is investigated to reduce misranking in the procedure of identifying and ranking critical contingencies among all the possible system contingencies.

Contingency Effects Analysis The critical contingency effects analysis is the most important but computationally demanding procedure in bulk power system reliability assessment. Most existing contingency effects analysis algorithms are implemented based on the traditional power flow model, which suffers from a lack of realistic system models and slow convergence or even divergence when the system is severely stressed. In this research, a non-divergent optimal quadratized power flow (NDOQPF) algorithm based on the quadratized power flow model is proposed for performing contingency effects analysis efficiently and realistically. In the proposed NDOQPF algorithm, the quadratized power flow model is adopted as a basis to achieve faster convergence in solving power flow solutions for the purpose of improving algorithm efficiency, and major system controls, such as real power economic dispatch, reactive power proportional dispatch, and remedial actions, are incorporated into the procedure of simulating the system response to critical contingencies for the purpose of achieving a realistic contingency effects analysis. This algorithm also aims to guarantee the convergence of power flow solutions under all system operating conditions such that an optimal or suboptimal solution can always be provided to operators. In addition, the proposed algorithm can effectively simulate 
system operating conditions in deregulated power systems.

Protection System Hidden Failure Analysis Protection systems are generally assumed to be perfect in current bulk power system reliability assessment practice. To account for the effects of hidden failures in the protection system on bulk power system reliability in this research, we incorporate the breaker-oriented substation model, which includes a specific bus arrangement and associated protection system schemes in the bulk power system network model. Based on such a detailed system network model and the analysis of the influence of the advanced system monitoring function on detecting protection system hidden failures, a protection system hidden failure effects analysis technique is developed to obtain hidden failure outages following any initial equipment outages. The proposed security-constrained adequacy evaluation methodology is extended so that it includes contingencies resulting from hidden failure outages. As a result, the effects of protection system hidden failures on bulk power system reliability can then be evaluated.

\subsection{Thesis Outline}

This thesis is organized as follows. Chapter 2 provides a literature survey of existing reliability modeling and assessment techniques for bulk power systems. This chapter summarizes the major work that has been done in this area and analyzes the advantages and disadvantages of existing techniques, which provides the motivation for this research.

Chapter 3 proposes a comprehensive approach for bulk power system reliability assessment. This chapter describes the overall algorithm of the proposed security- 
constrained adequacy evaluation methodology and the development of various advanced techniques used to implement this methodology, including the quadratized power flow model, the performance index approach based on system state linearization, the windchime contingency enumeration scheme, remedial actions, the non-divergent optimal quadratized power flow algorithm, and so on.

Chapter 4 evaluates the effects of protection system hidden failures on bulk power system reliability. This chapter introduces the background of protection system hidden failures, incorporates the breaker-oriented substation model into the system network model, analyzes the ability of advanced system monitoring to detect protection system hidden failures, and then develops the effects analysis procedure of protection system hidden failures. In addition, this chapter extends the bulk power system reliability evaluation framework proposed in the previous chapter to evaluate system contingencies caused by protection system hidden failures.

Chapter 5 applies the proposed comprehensive bulk power system reliability assessment approach to IEEE reliability test systems and then provides representative simulation results to demonstrate the effectiveness of the proposed approach.

Finally, Chapter 6 summarizes the results of this research and suggests topics for future study. 


\section{CHAPTER II \\ OVERVIEW OF BULK POWER SYSTEM RELIABILITY ASSESSMENT}

\subsection{Introduction}

This chapter provides an overview of various existing reliability modeling and assessment techniques for bulk power systems. The purpose of this overview is to summarize the main developments in the area of bulk power system reliability assessment in the past several decades and reveal the motivation of this research.

\subsection{Bulk Power System Reliability Modeling Methods}

Bulk power system reliability modeling techniques have evolved from traditional deterministic modeling methods to the current more advanced probabilistic modeling methods. Recently, some intelligent concepts, such as fuzzy set theory, have also been incorporated into probabilistic modeling techniques. 


\subsubsection{Deterministic Method}

It is incumbent on power system planners and operators to ensure that customers receive adequate and secure energy supplies within reasonable economic constraints. Historically, such a task has involved the assessment of bulk power system reliability using deterministic criteria [18, 19], which generally include a list of empirical contingencies involving the outages of some important power system components. With these contingencies in mind, planners and operators of the power system can incorporate sufficient redundancy so that any system failures during such contingencies can be prevented. The more comprehensive the list of contingencies, the lower the probability of a system failure resulting from contingencies not listed. Through such deterministic methods, a satisfactory degree of system reliability has been achieved in the past decades [4].

It is crucial that power system planners more closely examine the key element of deterministic criteria, i.e., the contingency list. The contingency list, which is generally developed based on the planners' experience, is often the result of an informal framework [4]. As power systems become more complex, and unforeseen power flow patterns occur because of independent power producers, the contingency list becomes a less reliable means of assessing the power system reliability because the impact of contingencies absent from the list may become significant [20]. Therefore, the trend is to develop automated procedures for selecting the contingency list by taking into account power flow patterns and new operating scenarios. 


\subsubsection{Probabilistic Method}

As power systems grow much larger and more complex, the need for the rigorous analysis of bulk power system reliability becomes more obvious. Because of the stochastic nature of system behavior, such as component outages or load-level changes, the development and application of probabilistic techniques for modeling the bulk power system and evaluating an appropriate set of expected risk indices have received considerable attention [2-4].

In the probabilistic modeling method, uncertainties affecting power system reliability are accounted for using probabilistic techniques. For example, the Markov model is widely used to represent component states, electric load levels, system states, and so on. Such a probabilistic modeling method enables the calculation of probability, frequency, and duration indices of system failures. These indices are no longer deterministic values but expected values of probability distributions [2, 3].

\subsubsection{Fuzzy-Probabilistic Method}

In recent years, some power system reliability probabilistic modeling methods have attempted to incorporate the fuzzy set concept in modeling system uncertainties [21-26]. For example, fuzzy numbers are used to model uncertainties in system component failure and repair rates, fuzzy load duration curves are developed using a fuzzy number in each time step [21-24], and fuzzy power flow models [25, 26] are developed to identify possible system behaviors given specified uncertainties. These fuzzy representations of 
system uncertainties are then integrated into the probabilistic evaluation procedure. However, by using this fuzzy-probabilistic method, the computational burden increases significantly without a commensurate gain in the quality of results.

\subsubsection{Summary}

Although the deterministic method still dominates the planning and operating phases in practice, it can no longer assess the reliability of modern complex power systems effectively. Instead, with the availability of more component failure data and computational resources, the more superior probabilistic modeling method becomes the promising means to represent practical system behavior. In the next section, bulk power system reliability assessment techniques are reviewed, with an emphasis on those based on probabilistic models.

\subsection{Bulk Power System Reliability Assessment Methods}

Current bulk power system reliability assessment techniques in the literature fall into two fundamental categories: analytical and Monte Carlo simulation techniques [27-31]. 


\subsubsection{Analytical and Monte Carlo Simulation Techniques}

The reliability assessment of bulk power systems must consider a multiplicity of factors, such as the failure and repair rates of equipment and operating practices, including economic generation scheduling, security controls, emergency controls, projected load variations, and maintenance schedules. These system characteristics can be represented with analytical models in the analytical technique [2, 4, 32]. By using the analytical technique, system contingencies, which may involve line failures, unit outages, or both, are first enumerated up to a certain level. To identify the contingencies that result in system failures, failure effects analysis is then conducted to test system contingencies against some predetermined criteria. The impact of each contingency on the system, such as line loading and bus voltages, is obtained by solving power flows. Based on the results of effects analysis, system reliability indices such as loss-of-load probabilities, frequencies, and durations can be calculated [33, 34].

The bulk power system reliability assessment problem is treated as a series of experiments by the Monte Carlo simulation technique. This technique consists of randomly sampling system states, testing them for acceptability, and aggregating the contribution of loss-of-load states to the reliability until the variations of reliability indices drop below pre-specified tolerances [3]. The basic approach can be applied for each hour in a year in chronological order (the sequential approach), or the hours of the study time can be considered as random (the random approach). The simulation of selected system states is done with the use of load flows that consider generation dispatch and other pre-selected operating policies. Simulation results are distributions of the 
variables of interest (i.e., circuit flows, voltage levels, energy curtailment, and so on). These results are utilized in the computation of appropriate reliability indices [35-37].

\subsubsection{The Comparison of Two Techniques}

The comparison of analytical and Monte Carlo simulation techniques is performed in this section. Both techniques solve power flow problems to identify system deficiencies. Power flow models vary widely and differ in computing speed, precision, and computer storage requirement. Since the number of contingencies in a practical system is enormous, a large number of power flow problems have to be solved. Therefore, in both analytical and Monte Carlo simulation techniques, the employed power flow model plays an important role in assessing overall system reliability efficiently and accurately [4]. On the other hand, the analytical and the Monte Carlo simulation techniques differ with regard to the processes of selecting states and evaluating reliability indices. The analytical technique selects states in an increasing order in terms of contingency levels, and this process stops at a particular component outage level or when the state probability becomes less than a specified value. Each state is assessed only once, and reliability indices are calculated mathematically from the statistical data associated with each state. Monte Carlo simulation techniques, however, select states randomly based on the concept of random numbers. States with greater probabilities of occurrence are more likely to be simulated several times, and this simulation process stops either after a fixed number of simulations or on the basis of statistical stopping rules. The expected values of indices are determined by averaging the indices obtained from each simulation [3]. 
In Monte Carlo simulation, the number of system states that must be sampled before the indices stabilize is extremely large, and this number increases significantly as the system reliability level increases. Thus, a major drawback of the Monte Carlo simulation method is that it requires a long computation time. In particular, when using the sequential approach, the computational burden may be unmanageable for a practical bulk power system. Besides the long computation time, another limitation exists in the random approach. In power systems, the outages of some components may influence the probabilities of related component outages, which are referred to as "fat tail" effects. Since the random approach does not include this sequence information, it cannot account for the fat tail effects in the simulation procedure. Compared to Monte Carlo simulation, the analytical technique can provide reliability indices in a relatively short computation time. It can also consider fat tail effects and other complex processes of the system using appropriate analytical models without significantly increasing the computational burden. In addition, the computational effort in the analytical method is much less dependent on the system reliability level.

\subsection{Adequacy and Security Reliability Assessment}

Bulk power systems must not only provide adequate generation and transmission capacity to meet the demand of customers while satisfying system operating constraints, but also be capable of maintaining system stability following faults, switchings, and other disturbances. Regarding these two aspects, the reliability of a bulk system can be 
evaluated by two attributes: adequacy and security $[2,39]$. Adequacy refers to the ability of the system to supply the aggregate electric energy requirements of customers within component ratings and voltage limits when planned and unplanned component outages occur. Adequacy assessment involves system steady-state conditions of postcontingencies, i.e., the system is assumed to always reach a stable equilibrium point after equipment outages, and the dynamics of the transition from one state to another are neglected. The second attribute, system security, refers to the ability of the power system to withstand disturbances arising from faults or equipment outages. Security assessment involves system transient responses and cascading sequences after a disturbance. Transient responses include the fluctuations of both the system frequency and bus voltages. If the fluctuations exceed certain operating limits, cascading sequences, such as line and generator tripping, may occur and persist until the system completely separates or collapses. These effects may not be properly accounted for in adequacy studies and must be captured in security evaluations. Although most research has been devoted to the adequacy assessment of bulk power system reliability, some concerns have recently shifted to security evaluations that also integrate the adequacy studies. The following two sections summarize the main approaches developed in these two areas.

\subsubsection{Adequacy Assessment}

The major difficulty in adequacy assessment involves the enormous computational effort required to analyze as many contingencies that may have a nonzero contribution to system unreliability as possible. The massive computational demand is a result of the 
following two factors: The first involves the large system size and the resulting large number of system states that must be assessed. It is usually not feasible or even possible to investigate all the contingencies of a power network. In practice, only credible outage states up to a certain contingency level are investigated. It has been noticed that as the system size increases, the consideration of higher-level contingencies is necessary, which will rapidly increase the computation time [4]. Another factor involves the complexity of the failure effects analysis in the assessment of each state. The objective of such an analysis is to identify the impact of each state on system operations by solving power flow problems. It is the most important but the most computationally demanding part of reliability assessment, and its performance influences the effectiveness of the overall reliability evaluation [2, 4].

Research attempting to solve the above two problems are further classified as adequacy and security-constrained adequacy evaluations, which are reviewed separately as follows.

\subsubsection{Adequacy Evaluation}

The following research effort based on analytical techniques has been dedicated to reducing the number of contingencies that must be evaluated and therefore to cut down the computational burden while at the same time increasing the assessment accuracy.

Inclusion of State Effects without Actual Evaluation To improve reliability assessment, a state extension algorithm is proposed [40, 41]. In this method, the system state space is enumerated in the form of a tree graph based on different outage levels, and 
a coherent system is assumed, i.e., if an investigated state is a failure state, the descendant states with higher outage levels in the sub-tree of this investigated state are concluded to be failures, too. The information of the sub-tree, including its probability and associated repair and failure rates, is computed based on the knowledge of the investigated state. The contribution of states in the sub-tree to system unreliability is taken into account when system reliability indices are calculated. In this method, the effects of some uninvestigated high-level contingencies are included in the reliability evaluation procedure, and more accurate indices are obtained without increasing the computational burden.

The drawback of this algorithm is that it requires the component sequence adjustment in the tree graph so that it includes as many effects as possible of un-investigated highlevel failure states. In its application to the testing systems [40], results show that it could not provide satisfactory assessment in some situations. In addition, for a large system, even the number of contingencies up to the boundary outage level is too large to be examined one by one [40]. A possible solution is to use the contingency selection scheme, which provides information useful in adjusting the component sequence to further reduce the computational effort in evaluating the states up to the boundary outage level.

Simplification of the System Model by System Equivalent Models The practical bulk power system can be extremely complex because of the large number of components and complex interconnections among them. To simplify the system model, an adequacy equivalent method [42, 43] is proposed, in which a general power network is divided into two areas: the area of interest (AI) and the interconnected area (IA). The IA is replaced with an equivalent model that is simpler than the original network but retains all the 
essential information. The equivalent model is expressed in terms of a capacity margin table that contains the information of available capacities, probabilities, and frequencies of simplified states in the IA. Once this equivalence is developed, the adequacy evaluation can be conducted effectively with reduced computational effort. In [44, 45], the equivalent method is extended to include the equivalent load model in the IA so that its load curtailment can also be considered. Furthermore, in [46], the composite power system is divided into three areas: the area of interest, the boundary buses, and the external area. The external area is replaced by an equivalent probabilistic model, and the statistical behavior of its components is included.

Although the system equivalent method can simplify the analysis to some extent and reduce the computational burden, it should be noted that this method has some limitations. First, an equivalent model is a simplified model of the original network and cannot be complete and precise in all aspects. In addition, this method is system dependent, i.e., different equivalent models need to be developed under different system operating conditions and for different areas of interest. As a result, the extra computational effort is brought into the evaluation procedure. Furthermore, the implementation of this method is based on the DC power flow model, which cannot check the voltage and reactive power of the power supply at load points and capture the effects of voltage and reactive power supplies on system reliability.

Reduction of State Space by the Contingency Selection Method In adequacy assessment, an important fact is that many component outages (contingencies) do not cause the service curtailment or other adverse impact on service quality [47-49]. The contingency selection method can eliminate states whose impact on the system is small, 
thus reducing the state space to include only states whose impact on the system is significant. Selected states are further ranked according to their levels of impact on the system [52]. Typical contingency selection methods consist of the performance index (PI) method, the screening method, and the hybrid scheme.

In the PI method, a variety of performance indices, such as the circuit current index, the voltage index, and the reactive power index, are defined to measure the normality of a system [51-55]. When a contingency occurs, the system operating condition changes and so do the associated PIs. The variations of PIs from pre-contingency to post-contingency can be considered as an indication of the impact of the contingency on system operations. Contingencies are further ranked in a descending order of the projected PI changes. Although the PI method is efficient, it is vulnerable to misrankings. The inaccuracies of the PI method are mainly the result of (1) the nonlinearities of the system model [55] and (2) the discontinuities of the system model arising from generator reactive power limits, regulator tap limits, and so on $[53,54]$. In contrast to the PI method, the screening method is more accurate but inefficient. In this method, the contingency selection is based on approximate network solutions such as Fast Decoupled Power Flow solutions $[54,56]$, which can consider the nonlinearities of the power system to some extent and provide more accurate results than the PI method. However, the contingency selection using the screening method needs to solve post-contingency cases, which are time consuming. In particular, time may be wasted while solving contingencies without much impact on system operations. The hybrid scheme [5, 50], a combination the PI and the screening methods, achieves both efficient and accurate contingency selection and ranking. Efficiency is achieved by employing the PI method first to quickly identify the 
conceivable contingencies. The accuracy of ranking is guaranteed by using screening methods for only a subset of contingencies that cannot be ranked with confidence by the PI methods. Thus, the hybrid scheme employs the best properties of both the PI and the screening methods.

The research reviewed above is based on the analytical technique. Because of the critical limitations of the Monte Carlo simulation technique, such as long computation time, its application is less popular than that of the analytical technique. To accelerate the convergence of the Monte Carlo simulation, some methods, including parallel processing [57-62], state space pruning [63-69], variance reduction [70-77], and so on, are proposed in the literature. The improvements, however, are quite limited, so the need persists for techniques that alleviate the computational burden while dealing with composite systems of realistic dimensions [38].

\subsubsection{Security-Constrained Adequacy Evaluation (SCAE)}

In the previous section, the main research effort focused on developing techniques that select credible contingencies. As far as effects analysis is concerned, traditional power flow techniques, including DC power flow and AC power flow, are generally used as the primary means to perform contingency evaluations.

Some recent research efforts have concentrated on improving effects analysis

performance by integrating remedial/corrective actions, i.e., remedial actions are applied to satisfy system security constraints while solving power flow problems after contingencies. This strategy is referred to as security-constrained adequacy evaluation 
(SCAE). System security constraints include bus voltage limits, line flow limits, real power and reactive power generation limits, and so on. In practice, when generator or line outages occur or line overloads resulting from outages occur, generating units should be rescheduled so that the power system can maintain generation-demand balance and alleviate line overloads. If violations corresponding to the bus voltage limits exist, reactive power (generation, transformer taps, and shunt compensation) must be rescheduled to eliminate abnormal voltages in the system [78]. The purpose of these remedial actions is to keep the system operating normally, and thus avoid load curtailment, if possible, or to minimize total load curtailment, if unavoidable.

In [78-84], some security-constrained adequacy evaluation methodologies are presented. In these methods, the procedures for effects analysis are implemented by solving power flow problems coupled with remedial actions. Optimization power flow problems are formulated to minimize the load curtailment through remedial actions and load shedding in the effects analysis procedure. Linear programming models for remedial actions that adjust active power [79-81] and reactive power [83] are derived. Some techniques, including the simplex method and the interior point method [78], are applied to solve the formulated optimal power flow problems.

Since the optimal power flow technique is an important issue related to the effects analysis procedure in SCAE, a brief review of the formulation and the solution of optimal power flow is provided in the following section. 


\subsubsection{Optimal Power Flow}

Optimal power flow (OPF) is a mathematical optimization tool for adjusting the power flow in a power network to achieve the optimal value of a predefined objective while satisfying system operating constraints [85, 86]. OPF has undergone intensive research and development over the past several decades [87-95]. Mathematically, the general OPF problem can be expressed as follows.

Minimize $f(x, u)$

Subject to $g(x, u)=0$

$$
\begin{aligned}
& h(x, u) \leq 0 \\
& u^{\min } \leq u \leq u^{\max },
\end{aligned}
$$

where

$f(x, u)$ objective function

$g(x, u)=0$ power flow equations

$h(x, u) \leq 0$ operating constraints

$x$ vector of system state variables

$u$ vector of control variables.

$u^{\min } \leq u \leq u^{\max }$ feasible region of control variables.

The OPF objective function varies with different operational objectives. Typical examples of objective functions are minimum generation cost [96, 97], minimum system transmission loss [98], voltage and reactive power optimization [99], preventive and corrective control optimization [100], and so on. In reliability assessment, the objective may include the minimum amount of remedial actions, minimum load curtailments, and 
so on. A predefined objective can be achieved if control variables in the system are available for adjustment. Control variables usually include MW/MVAR generation adjustment, shunt capacitor/reactor switching, phase shifter adjustment, transformer tap adjustment, load transfer, area interchange, and load shedding.

Constraints in the OPF generally contain equality and inequality equations. Equality constraints are usually power flow equations. Inequality constraints consist of functional operating constraints, including branch flow limits, bus voltage magnitude limits, and so on. In addition, the feasible region of control variables is contained in the constraints set, including unit active and reactive power output limits, transformer tap limits, and so on.

Some variations in formulating the OPF problem exist with its application in different areas. These variations mainly include the decomposition of real and reactive OPF [86], contingency constrained OPF [101, 102], stability constrained OPF [103, 104], and so on. Also, a wide variety of optimization techniques, such as linear programming (LP), quadratic programming, nonlinear programming, hybrid versions of linear programming, and integer programming, have been employed to solve the formulated OPF problems. Because of the large-scale nature of the problem and the resulting computational complexity of reliability assessment, the linear programming (LP) technique, which has a relatively simple formulation and is capable of providing fast solutions, is the most attractive tool.

The application of the LP technique requires that the nonlinear objective function and constraints be linearized around the operating point. The obtained LP problem can be solved using the different variants of the simplex method. Among them, the most commonly used technique is the revised simplex method. However, interior point 
methods have recently shown superiority over the simplex method for solving large-scale LP problems.

In spite of the tremendous research that has been done, the OPF remains a difficult mathematical problem, and its use has been somewhat restricted by its lack of efficiency, flexibility, and reliability. To solve such problems, a novel formulation and solution approach for the OPF problem was developed [86]. The optimal solution is obtained by gradually reducing the power mismatch at each bus while at the same time incorporating the economic dispatch process and observing operating constraints. This OPF model has the following merits: (1) it guarantees a non-divergent solution if a feasible power flow solution exists; (2) it realistically simulates actual system operating practices by incorporating the economic dispatch and remedial actions when operating constraint violations occur; and (3) compared with other OPF models, it is efficient because of the implementation of extensive model reduction and the application of sparse techniques [86].

\subsubsection{Integrated Adequacy and Security Assessment}

The security assessment of a composite power system involves evaluating system behavior while integrating transient stability as well as cascading sequences after a disturbance. For the correct assessment of system security, the contingency analysis has to be repeated for all significant initial conditions and at different points in time to account for the impact of time-dependent factors, including the transient behavior of generators, the operation of many types of protection schemes, the automatic actions of 
different controls, and operators' actions [105, 106]. Nowadays, a major effort in the security assessment has been devoted to extending existing adequacy assessment techniques to include the assessment of system security. Other work is based on time domain simulation that achieves the security assessment.

Integration of Transient Stability Limit: A framework that evaluates both the adequacy and security reliability of the system is presented in [106]. In security analysis, the system transient stability limit is identified by comparing the fault clearing time with the critical clearing time. Providing critical clearing time requires a transient stability evaluation. For unstable states, remedial actions are applied, and some indices corresponding to the security evaluation are also provided. The security assessment presented in reference [105] also takes into account the cascading sequences besides the transient stability. System states are classified into nine types: adequate, inadequate, partially adequate, stable, unstable, secure, not secure, marginally adequate, and system collapse states. Based on the classified states, a possible sequence of events after the occurrence of a disturbance is built.

Integration of Security Constraints The security assessment technique presented in [81, 82] is based on the previously described security-constrained adequacy evaluation. In [81], besides the basic operating constraints that have to be satisfied for the steady- state performance, a transient-performance constraint set that can reflect the transient behavior of a power system when subject to system faults is formed. In [82], instead of introducing new constraint sets, dynamic system models are suggested for use in determining system operating limits that include security consideration. 
Design Contingency Set In [108], a design contingency set (DCS) is set up first. A DCS is a set of contingencies that the system must be able to withstand with acceptable transient performance. Based on the DCS, security limits are derived by ensuring that the system is stable for all DCS contingencies. Security evaluation can be quantified by evaluating the ability of the system to operate within the security limits in the steady state. This method captures the impact of contingencies on security without the need for a transient analysis. However, it may result in the following two problems: (1) because reliability assessment is based on the DCS, it is difficult to determine if the DCS was selected properly and (2) the procedure of determining security limits still requires stability studies.

Time-Domain Simulation In [107], a time-domain simulation that obtains the power system response after a disturbance is applied. In the simulation, the dominant dynamic phenomena, i.e., the voltage stability problem, power-frequency control, and the cascading tripping of power system components, are crucial because these processes determine whether new steady-state equilibrium can be reached. The consideration of voltage stability in reliability assessment is addressed in [109-112]. After the power system reaching a steady state, the procedure of minimizing the overall curtailed load, voltage restoration, and economic dispatching is applied.

Because of the complexity of considering transient stability as well as cascading events after contingencies, security assessment incurs an extremely heavy computational burden and has slow convergence. 


\subsubsection{Summary}

Bulk power system reliability assessment includes two aspects: adequacy and security.

Of the two, adequacy assessment has been the major concern. The key issue associated with adequacy assessment is the computational burden and the performance of effects analysis to evaluate large-scale systems. Research, which has focused on both analytical and Monte Carlo simulation techniques, has found the former superior to the latter. In addition, a security-constrained adequacy evaluation has recently received more attention, as it is able to simulate actual operational practices that improve the performance of effects analysis procedures. Regarding security assessment, some effort has been devoted to including transient stability analysis and cascading sequences of power system behavior after disturbances occur.

\subsection{Summary}

The overview of current bulk power system reliability assessment techniques reveals following facts: (1) the probabilistic modeling method has become the most promising means to represent practical system behavior, (2) the analytical technique is superior to the Monte Carlo simulation technique in performing reliability assessment for large scale bulk power systems, and (3) the contingency selection method and the securityconstrained adequacy evaluation method are the most effective ways to reduce the system state space and perform contingency effects analysis, respectively. 
This overview also indicates that (1) contingency selection and ranking based on the current contingency selection method is prone to misranking, (2) the security-constrained adequacy evaluation method based on the traditional power flow model suffers from the lack of realistic system simulation and slow convergence (even non-convergence) when the system is heavily stressed, (3) no research effort has been devoted to the evaluation of the effects of protection system hidden failures on bulk power system reliability assessment. In this work, these problems are addressed in the proposed comprehensive approach for bulk power system reliability, which is illustrated in detail in following Chapter III and Chapter IV. 


\section{CHAPTER III \\ BULK POWER SYSTEM RELIABILITY ASSESSMENT METHODOLOGY}

\subsection{Introduction}

The objective of this research is to advance the state of the art in bulk power system reliability assessment. Specifically, a framework of security-constrained adequacy evaluation (SCAE) based on the analytical technique is proposed to determine the ability of the bulk system to supply the electric load while satisfying security constraints. The SCAE framework is implemented based on a single-phase quadratized power flow model and encompasses three main steps: (a) critical contingency selection, (b) contingency effects analysis, and (c) reliability index computations. To address the major challenges in the bulk power system reliability assessment practice, this research focuses on the first two steps.

Since a complete evaluation of all possible system contingencies is impractical, only critical contingencies, those that may lead to system unreliability, are identified in a rapid preliminary analysis for the purpose of reducing the system state space. In the critical contingency selection, a systematic contingency selection/enumeration scheme based on the performance index approach, the single-phase quadratized power flow model, and the wind-chime contingency enumeration scheme is investigated to improve the accuracy of the critical contingency selection. 
Contingency effects analysis is the most important but the most computationally demanding procedure. In the effects analysis, it is important to simulate contingencies realistically to capture the system response under all major controls and adjustments. The simulation of contingencies must also be efficient so that the overall computational effort of the SCAE methodology is reasonable. Effects analysis based on traditional power flow technology often breaks down (non-convergence), especially when multi-level contingencies are considered and when the system is severely stressed. Thus, in this proposed research, a non-divergent optimal quadratized power flow (NDOQPF) algorithm is proposed for effects analysis. This algorithm is implemented based on the single-phase quadratized power flow model because of its faster convergence and ability to model complex component characteristics. A constrained optimization problem is formulated that can incorporate system security constraints and remedial actions in the contingency simulation. The non-divergence is achieved by the introduction of fictitious bus injections that are driven to zero as the solution progresses. The proposed algorithm guarantees convergence if a solution exists; in the event that a solution does not exist, it provides a non-optimal solution that may include load shedding. In addition, this algorithm is capable of efficiently solving the ISO/RTO operational model in the deregulated environment and providing a solution under all conditions.

While this research focuses on the critical contingency selection and contingency effects analysis, to form an overall reliability evaluation procedure, the SCAE framework also includes the reliability index computation. Reliability indices, including probability, frequency, and duration indices of system failure events, are calculated and considered as a quantitative indication of the level of system reliability. 


\subsection{The Overall Algorithm}

The overall developed computational algorithm of the proposed SCAE methodology for bulk power system reliability assessment, shown in Figure 3.1, is implemented based on the single-phase quadratized power flow model. The constituent parts of the approach are critical contingency selection, contingency effects analysis, reliability index computation, and so forth.

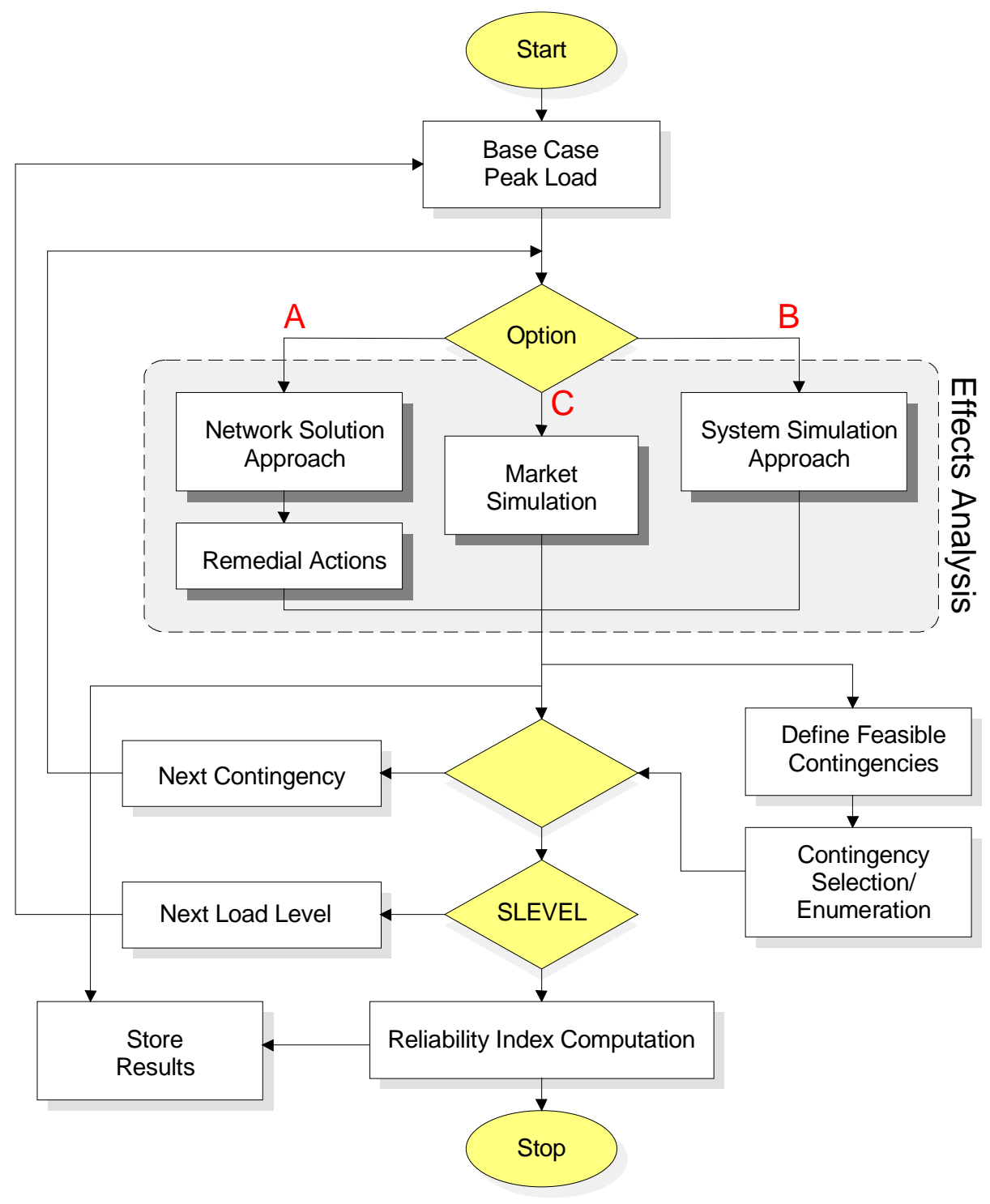

Figure 3.1: Overall computational algorithm of the SCAE methodology. 
The bulk power system reliability assessment procedure based on the proposed SCAE methodology and functions of different parts of the overall algorithm of the SCAE methodology are discussed below.

First, feasible contingencies up to a certain contingency level are defined, and an enumeration for each level of feasible contingencies is provided by the contingency enumeration scheme. Then, the contingencies in each level are ranked according to their impact on system operation by the contingency selection scheme. Such a contingency enumeration and selection procedure can be repeated for a number of electric load levels. The ranked contingencies are then subjected to the effects analysis.

Three options for the effects analysis are provided in branches A, B, and C: the network solution approach with possible remedial actions, the system simulation approach, and market simulation, respectively. These three options provide a means to perform the effects analysis of either regulated or deregulated power systems and to evaluate the adequacy or security aspect of system reliability.

In branch A of Figure 3.1, the network solution approach with remedial actions applies the proposed NDOQPF algorithm to the effects analysis of regulated power systems. It is implemented based on the single-phase quadratized power flow model. The contingency simulation procedure incorporates the major controls and adjustments inherent in a regulated environment. A constrained optimization problem is formulated to solve for the predefined operating objective. Whenever abnormal operating conditions occur, remedial actions are applied to satisfy security constraints. Non-divergence is achieved by introducing fictitious bus injections that are driven to zero as the solution progresses. If a 
solution exists, the proposed methodology guarantees convergence; if a solution does not exist, it provides a non-optimal solution that may include load shedding.

In recent years, electric power industry has been deregulated in many countries. The market simulation in branch C in Figure 3.1 represents a means to perform the effects analysis in deregulated power systems. In a deregulated environment, the ISO/RTO operational procedure is to determine the least expensive dispatch of generation based on energy bids. When transmission congestion occurs, appropriate remedial actions are taken to relieve congestion. This procedure, although has a different objective and remedial action set, is similar to that in the regulated environment. Therefore, the effects analysis in the deregulated environment can be formulated and solved by the NDOQPF methodology in much the same way as it is in the regulated system. Moreover, because of the impact of deregulation on system operation, the traditional power flow analysis under contingencies is prone to a non-convergence situation. Because of its ability to solve such a problem, the NDOQPF algorithm is especially effective in the deregulated environment to solve the ISO/RTO operational model by always providing a solution to ISO/RTO operators under all conditions.

The system simulation approach in branch B is capable of considering full security evaluation in the effects analysis, which includes transient stability analysis, cascading sequences, and other dynamic aspects of power system behavior after contingencies. This security assessment scheme is not addressed in this work, but it will be implemented in future work.

The results of the contingency effects analysis for all evaluated contingencies are stored and processed to provide reliability indices. Three classes of reliability indices are 
computed: (a) probability indices, (b) frequency indices, and (c) duration indices. These indices provide system planners and operators with quantitative values that indicate the level of system reliability.

The basis of the proposed SCAE methodology, i.e., the single-phase quadratized power flow model, and the three major constituent parts of the methodology, including critical contingency selection, contingency effects analysis, and reliability index computation, are described in detail in the following sections.

\subsection{The Single-Phase Quadratized Power Flow Model}

In the proposed SCAE methodology, an advanced power flow model, the single-phase quadratized power flow (SPQPF) model [52], is applied in both critical contingency selection and contingency effects analysis. The SPQPF model is able to improve the accuracy of the critical contingency selection and the efficiency of power flow solutions in the effects analysis. To illustrate the characteristics of the SPQPF model, a comparison of the traditional power flow (TPF) model and the SPQPF model is described below.

The TPF model consists of power balance equations at each bus in the system. These equations are expressed in polar coordinates in terms of system states (bus voltage magnitudes and angles); nonlinear trigonometric terms inevitably appear in the equations. In addition, most system loads are induction machines, and their models contain relatively high-order nonlinear terms. Consequently, the TPF formulation results in a set of complex and highly nonlinear equations. When an iterative method such as the 
Newton-Raphson method is used to solve such equations, a number of iterations may be required and in some cases, the solution may be difficult to find. [113].

The SPQPF model, however, is set up based on the application of the Kirchhoff's current law at each bus, with the intention that most of the power flow equations are linear in the large-scale system. Furthermore, system states (bus voltage phasors) are expressed as Cartesian coordinates (bus voltage real and imaginary parts) that can avoid trigonometric terms. As trigonometric functions are absent, power flow equations are less complex. Moreover, since the Newton-Raphson method is ideal for solving quadratic equations, all power flow equations are quadratized by introducing additional state variables. As a result, all power flow equations are linear or quadratic. The formulation of the quadratized power flow model provides superior performance in two aspects: (a) faster convergence and (b) ability to model complex load characteristics in the quadratized form.

\subsection{Critical Contingency Selection}

Since the complete evaluation of all possible system contingencies is impractical, critical contingency selection is applied first to approximate but quickly identify the critical contingencies that may lead to system unreliability, such as system loss of load. Such selection of critical contingencies is implemented using an improved contingency selection/enumeration technique based on the performance index (PI) approach, the single-phase quadratized power flow model, and the wind-chime contingency 
enumeration scheme, which can select critical contingencies with high efficiency and less misranking. This technique is developed in this section.

\subsubsection{The Performance Index Linearization Approach}

The system performance index, defined as a function of system states and a contingency control variable [52, 113], is used to forecast the influence of contingencies on the system operation based on pre-contingency conditions. The performance index is generally expressed as follows:

$J=\sum_{j} w_{j}\left(f_{j}\left(x, u_{c}\right)\right)^{2 n}$,

where

$x \quad$ system state vector

$w_{j}$ appropriate circuit weight coefficient, $0<w_{j} \leq 1$

$n \quad$ integer parameter that defines the exponent.

$u_{c}$ contingency control variable

Note that the value of contingency control variable $u_{c}$ is one (1.0) for the precontingency condition and changes to zero (0) for the post-contingency case.

The quantities $f_{j}$ inside the parentheses express the normalized value, such as power flow, circuit current, voltage magnitude, and generator reactive power, with respect to equipment capability or allowable limits. Thus, while the values of the quantities in the parentheses in the range (-1.0 to 1.0$)$ indicate the system normal operating conditions of the system, the values outside this range indicate the system abnormal operating 
conditions of the system. When these quantities are raised to a power of $2 n$, they will produce large values for abnormal conditions and very small values for normal conditions.

Two examples of performance indices, i.e., current-based circuit loading index $\left(J_{C}\right)$ and voltage index $\left(J_{V}\right)$, are defined as follows:

\section{Current-Based Circuit Loading Index}

$J_{C}=\sum_{j} w_{j}\left(\frac{I_{j}}{I_{N, j}}\right)^{2 n}$

where

$I_{j} \quad$ current magnitude in circuit $j$

$I_{N, j}$ current rating of circuit $j$

$w_{j}$ appropriate circuit current weight, $0<w_{j} \leq 1$

$n \quad$ integer parameter that defines the exponent.

\section{Voltage Index}

$J_{V}=\sum_{k} w_{k}\left(\frac{V_{k}-V_{k, \text { mean }}}{V_{k, \text { step }}}\right)^{2 n}$,

where

$V_{k} \quad$ voltage magnitude at bus $k$

$V_{k, \text { mean }}$ nominal voltage value (typically 1.0 p.u.), which is in general the mean value in the desired range, i.e., $\frac{1}{2}\left(V_{k}^{\max }+V_{k}^{\min }\right)$ 
$V_{k, \text { step }} \quad$ voltage deviation tolerance (i.e., $\left.\frac{1}{2}\left(V_{k}^{\max }-V_{k}^{\min }\right)\right)$

$w_{k} \quad$ appropriate bus weight, $0<w_{k} \leq 1$

$n \quad$ integer parameter that defines the exponent.

Performance indices provide a quantitative way of representing the impact of contingencies on system operating conditions. A contingency that causes a change in the system operating condition will be accompanied by a change in the performance indices ( $\Delta J$ ) before and after the contingency. Thus, the critical contingencies that may have an adverse impact on system reliability can be recognized by ranking the changes in the performance indices. The larger the performance index change $(\Delta J)$ is, the more critical the corresponding contingency is.

Generally, the post-contingency performance index (PI) or the change in the performance index $(\Delta J)$ before and after the contingency is found by linearizing the performance index in terms of the contingency control variable based on the precontingency condition, i.e.,

$$
\begin{aligned}
& J^{\text {post }}\left(u_{c}=0\right) \cong J^{\text {pre }}\left(u_{c}=1\right)+\frac{d J}{d u_{c}}\left(u_{c}-1\right), \\
& \Delta J=J^{\text {post }}\left(u_{c}=0\right)-J^{\text {pre }}\left(u_{c}=1\right)=-\frac{d J}{d u_{c}} .
\end{aligned}
$$

Such a performance index linearization method only takes into account only the firstorder term with respect to contingency control variable $u_{c}$ when calculating $\Delta J$. This method is prone to misranking because of the inaccuracies caused by system nonlinearities. To reduce the error introduced by the linear approximation in the general performance index linearization method, a new technique that linearizes system states 
with respect to the contingency control variable is investigated to reduce the incidence of misranking. The system state linearization approach, implemented based on the quadratized power flow model, can include higher-order (nonlinear) terms in calculating performance index changes to reduce the error.

\subsubsection{The System State Linearization Approach}

In the system state linearization approach $[114,115]$, instead of linearizing the performance index directly, the system states of the quadratized power flow model are linearized with respect to the contingency control variable. The post-contingency performance index $J$ is then calculated based on the approximate post-contingency system states as follows:

$$
J=J\left(x^{0}+\frac{d x}{d u}\left(u_{c}-1\right), u_{c}\right)
$$

where

$x^{0}$ pre-contingency operating conditions

$x \quad$ system states of the SPQPF model

$u_{c}$ contingency control variable

The use of linearized system states in calculating the system performance index includes higher-order terms in Taylor's series and provides traces of indices with curvature, which can follow the highly nonlinear variations of the original performance indices to some extent. Based on Equation (3.6), the change in performance index ( $\Delta J$ ) is calculated as follows: 
$\Delta J=J^{p o s t}\left(x^{0}+\frac{d x}{d u_{c}}\left(u_{c}-1\right), u_{c}\right)-J^{p r e}\left(x^{o}, u_{c}=1.0\right)$

The sensitivity of the state with respect to the control variable can be easily computed as follows:

$$
\frac{d x}{d u_{c}}=-\left[\frac{\partial G(x, u)}{\partial x}\right]^{-1}\left[\frac{\partial G(x, u)}{\partial u_{c}}\right]
$$

Note that $\frac{\partial G(x, u)}{\partial x}$, the Jacobian matrix of the system, is pre-computed at the present operating condition and remains invariant for all contingencies. Thus, for each contingency, only the partial derivatives of the power flow equation $G(x, u)$ with respect to the contingency control variable have to be computed. The vector has only a few nonzero entries, so the computations are extremely fast. It should also be noted that $\frac{d x}{d u_{c}}$ is a vector of the same size as the state vector, each element of which is the derivative of the corresponding system state with respect to the contingency control variable. Once the new state is computed via this linear approximation, the calculation of the new value of the performance index is a straightforward operation.

The concept of this approach is presented graphically in Figures 3.2 and 3.3, based on results obtained from the application of the method to a test system. The first-order analysis curve represents the general linear curve obtained from the performance index linearization method. The higher-order analysis curve represents the nonlinear curve obtained from the system state linearization method.

It can be seen that the system state linearization approach is superior to the general performance index linearization method in calculating performance index changes and 
the subsequent ranking of contingencies. Thus, the system state linearization approach has unique potential for improving the contingency selection accuracy [113-117].

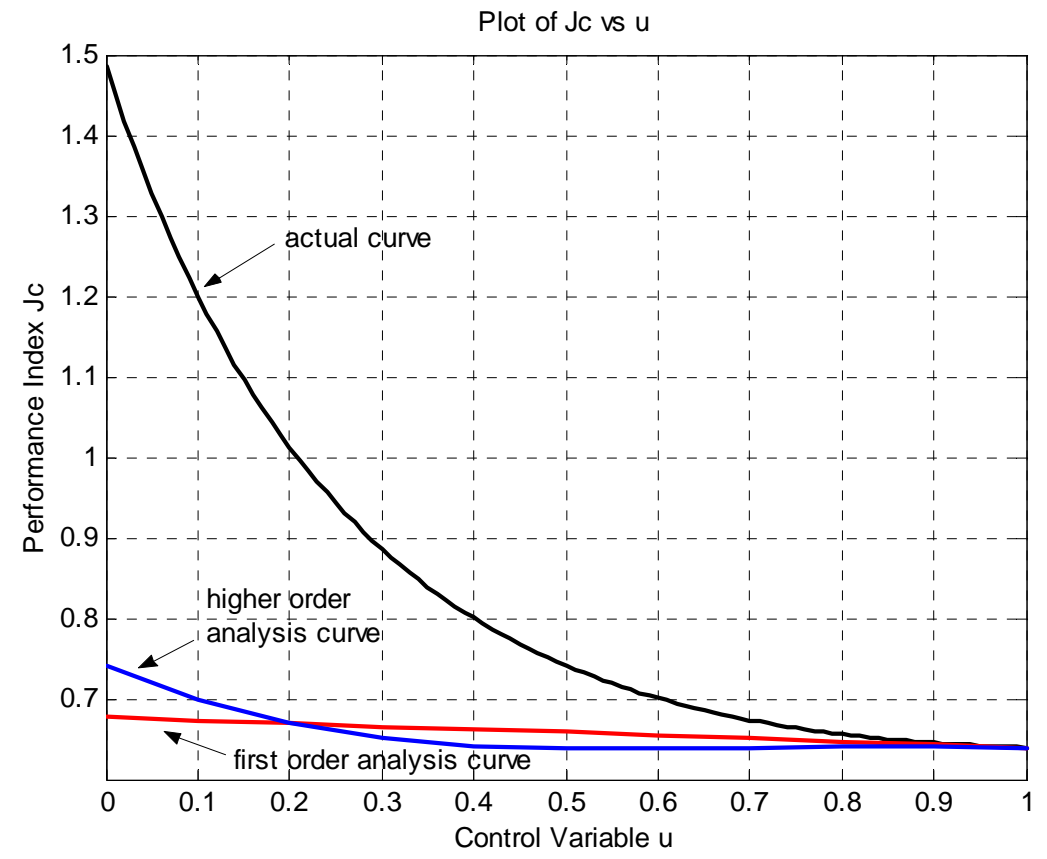

Figure 3.2: Plot of the circuit-loading index vs. the contingency control variable $\mathrm{u}_{\mathrm{c}}$.

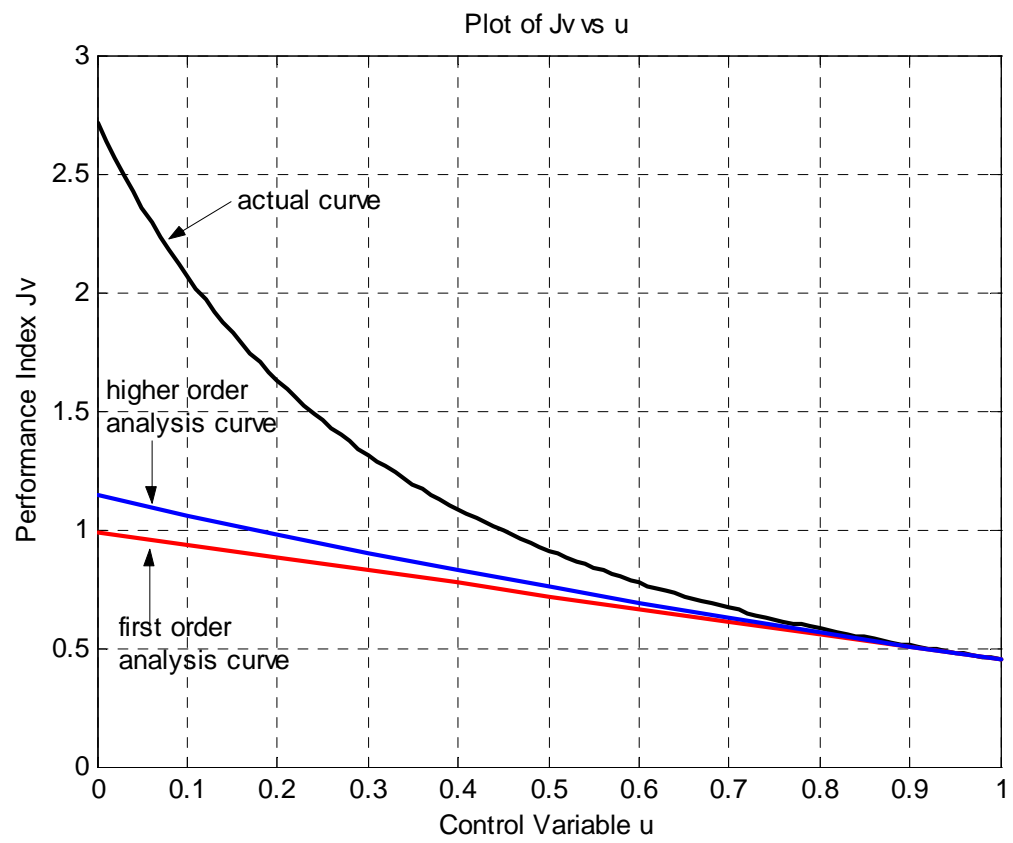

Figure 3.3: Plot of the voltage index vs. the contingency control variable $\mathrm{u}_{\mathrm{c} .}$ 


\subsubsection{The Wind-Chime Contingency Enumeration Scheme}

The wind-chime contingency enumeration scheme [33,114], shown in Figure 3.4, can systematically enumerate system contingencies according to their different outage levels and rank contingencies in each level in terms of severity based on the results of the performance index changes obtained from the system state linearization method.

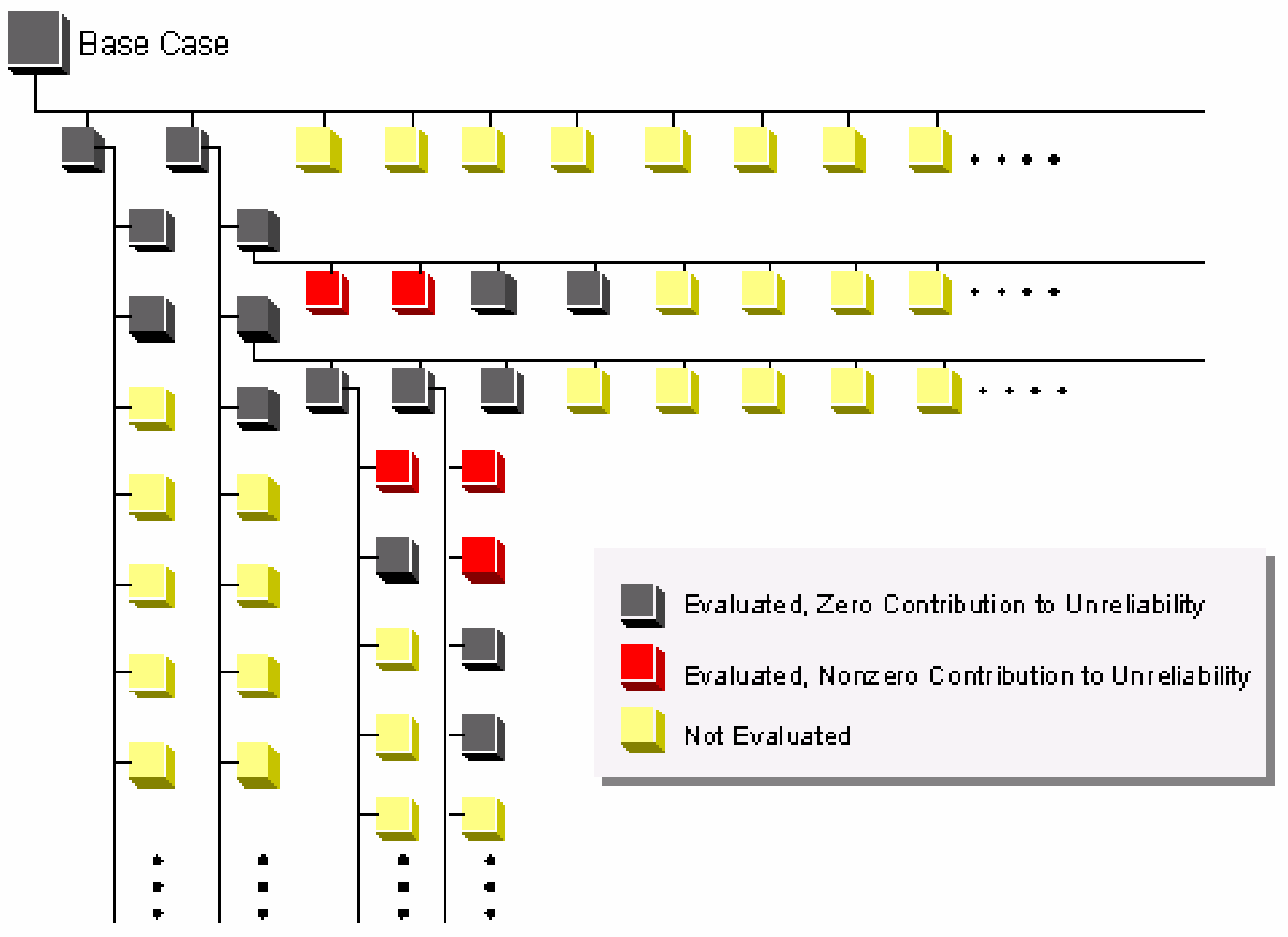

Figure 3.4: Wind-chime contingency enumeration scheme.

The contingency enumeration procedure begins with the base case. All the first-level contingencies are enumerated and ranked in decreasing order of severity. The second outage-level contingencies are obtained from each contingency in the first level by having one more component on outage and then ranked in the same way. The new outage 
component should be selected to make sure the obtained contingencies are distinct. This procedure continues until it reaches the predefined contingency depth level.

In each outage level, the reliability evaluation starts from the highest ranked contingencies in terms of severity. If the evaluation results of several successive contingencies show zero contribution to system unreliability, it is reasonable to conclude that the remaining contingencies in this level do not need to be investigated since they are considered to affect system reliability less severely. Figure 3.4 shows the resulting three types of system contingencies: (1) evaluated contingencies with nonzero contribution to unreliability, (2) evaluated contingencies with zero contribution to unreliability, and (3) non-evaluated contingencies. In such a way, computational effort can be saved by performing reliability evaluation only on the most severe contingencies.

\subsection{The Contingency Effects Analysis}

After critical contingency selection, the contingency effects analysis is performed to test selected system contingencies (states) against certain criteria to identify the contingencies that violate the criteria and therefore represent system failure. This procedure is the most important and computationally demanding in system reliability evaluation. In this section, a non-divergent optimal quadratized power flow (NDOQPF) algorithm is proposed for contingency effects analysis, as it can realistically and efficiently simulate the system response to critical contingencies and incorporate major system controls and adjustments such as real power economic dispatch, reactive power 
proportional dispatch, and remedial actions. For this algorithm, a constrained optimization problem is formulated to solve for the predefined operation objective while satisfying system security constraints. Non-divergence is achieved by introducing fictitious bus injections that are driven to zero as the solution progresses. If a solution exists, the proposed algorithm guarantees convergence; if a solution does not exist, it provides a sub-optimal solution that may include load shedding.

To consider the effects of remedial actions on system reliability, quadratized remedial action models are developed and incorporated into the effects analysis procedure. Both the quadratized remedial action models and the NDOQPF algorithm are discussed below.

\subsubsection{Remedial Actions}

Remedial actions (RAs) provide a means to correct abnormal system operating conditions, such as alleviating circuit overloads and abnormal voltages. Abnormal conditions usually result from scheduled or random events, particularly system contingencies. A list of typical remedial actions along with their relative costs is presented in Table 3.1.

Remedial actions greatly affect the reliability of power system operations by providing corrective measures after contingencies. In the SCAE methodology, whenever security constraint violations occur after a certain contingency, remedial actions without load shedding will be applied first. If the violations cannot be eliminated completely, the last type of remedial action, i.e., load shedding, would be used to alleviate the emergency. A contingency that requires load shedding to maintain normal system operation is recorded 
as a system failure. Such results of the contingency effects analysis are stored and subsequently used to calculate reliability indices.

Table 3.1: List of typical power system remedial actions.

\begin{tabular}{|c|c|c|}
\hline & Remedial Action & Associate Cost \\
\hline 1 & Shunt Capacitor Switching & Low \\
\hline 2 & Shunt Reactor Switching & Low \\
\hline 3 & Phase Shifter Adjustment & Low \\
\hline 4 & MVAR Generation Adjustment & Low \\
\hline 5 & Generation Bus Voltage & Low \\
\hline 6 & Transformer Taps & Low \\
\hline 7 & FACTS Controls & Low \\
\hline 8 & Load Transfer & Low \\
\hline 9 & MW Generation Adjustment & Moderate \\
\hline 10 & Area Interchange & High \\
\hline 11 & Interruptible Load Shedding & High \\
\hline 12 & Firm Load Shedding & High \\
\hline 13 & Critical Load Shedding & High \\
\hline
\end{tabular}

Quadratized remedial action models are set up to analyze the effects of remedial actions on power system reliability. A control variable $u$ is integrated into each remedial action model to represent the availability and magnitude of the remedial action [118120]. An example of a shunt capacitor/reactor model is provided below to illustrate the quadratized models of remedial actions. 
Figure 3.5 shows a discrete shunt switched capacitor/reactor model that is connected at bus $k$. It is characterized with the maximum admittance $\tilde{y}_{k, \max }$ and remedial action control variable $u_{S C R}$.

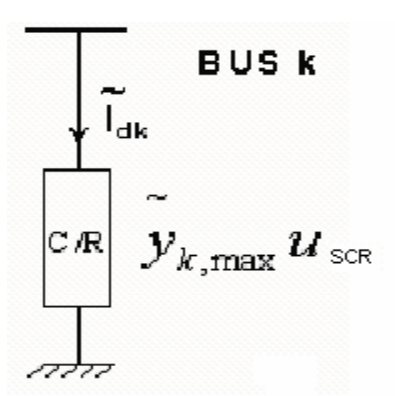

Figure 3.5: Shunt capacitor/reactor at bus k.

The control variable $u_{S C R}$ is defined as follows:

$u_{S C R}=\frac{i}{n}, \quad i=0,1, \cdots n$

where $n$ is the total number of switched steps and $i$ represents the different steps that the capacitor/reactor can be adjusted to. The value of $u_{S C R}$ indicates the available normalized amount of adjustment and varies between 0 - 1 .

Based on the defined control variable $u_{S C R}$, the model for this switched capacitor/reactor in standard form is given as follows:

$\tilde{I}_{d k}=\tilde{y}_{k, \max } u_{S C R} \tilde{V}_{k}$.

This model is already a quadratized model since the highest term order is no greater than two. Therefore, no effort is required to quadratize this model. For other remedial action models in which the highest term order is greater than two, additional state variables should be introduced to quadratize the models. 


\subsubsection{The Non-Divergent Optimal Quadratized Power Flow Algorithm}

In this section, a non-divergent optimal quadratized power flow (NDOQPF) algorithm is proposed for contingency effects analysis to simulate the system response to critical contingencies.

Consider an electric power system and let vectors $\mathbf{x}$ and $\mathbf{u}$ represent system states and available controls of remedial actions, such as unit real/reactive generation adjustments, switched capacitors/reactors, and so on. The last remedial action that would be used is load shedding. Assume a given initial operating state vector $\mathbf{x}^{0}$ and control variable vector $\mathbf{u}^{0}$ and consider a general system bus $k$ in the system, as shown in Figure 3.6. Unless $\mathbf{x}^{0}$ and $\mathbf{u}^{0}$ represent a power flow solution, a current mismatch equal to $I_{m k_{-} r}+j I_{m k_{-} i}$ exists at bus $k$. Assume that a fictitious current source is placed at bus $k$ and let the output of the current source be equal to $I_{m k_{-} r}+j I_{m k_{-} i}$. Taking into account the fictitious current source, Kirchhoff's current law is then satisfied at bus $k$. Similar fictitious sources/mismatches can be assumed for all other SPQPF equations such that $\mathbf{x}^{0}$ and $\mathbf{u}^{0}$ represent the current system operating condition. The actual operating condition of the system can be obtained by gradually driving the output of fictitious sources/mismatches to zero. This transition can be achieved along a trajectory that maintains feasibility and optimality. 


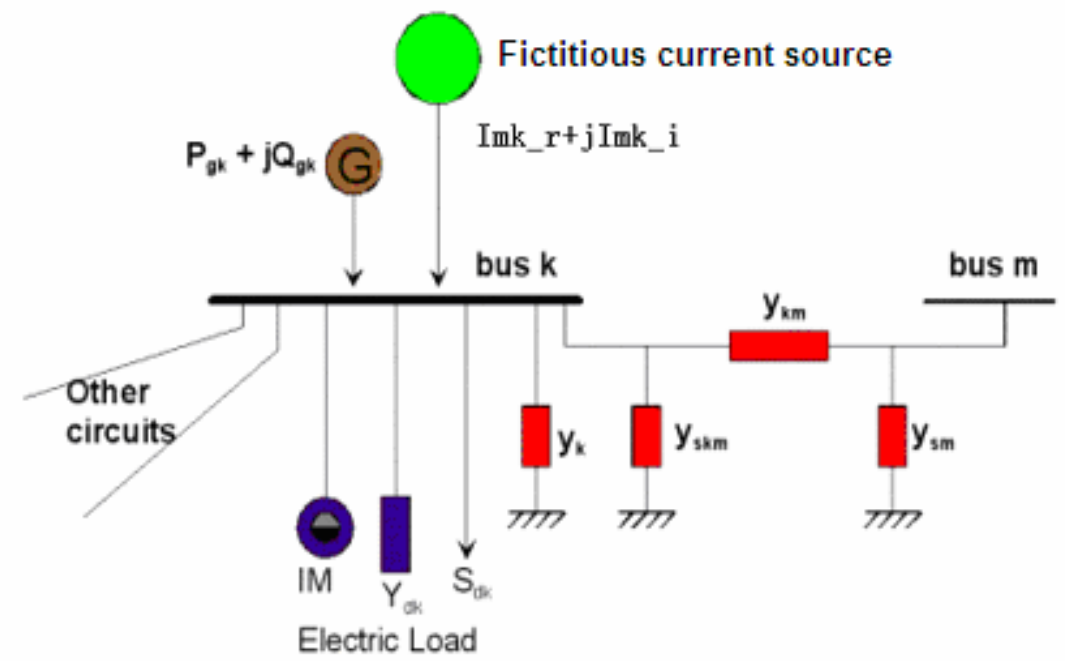

Figure 3.6: A general bus $k$ in a power system with a fictitious current source.

Mathematically, the above procedure is formulated as an optimization problem:

Minimize $\quad J=\mu \sum_{i=1}^{n}\left|m_{i}\right|+\sum_{j=1}^{r} \lambda_{j}\left|\Delta u_{j}\right|$

Subject to $G(\mathbf{x}, \mathbf{u}, \mathbf{m})=0$

$$
\begin{aligned}
& V_{j}^{\min } \leq\left|\tilde{V}_{j}\right| \leq V_{j}^{\max } \quad j=1, \cdots, b \\
& \tilde{I}_{j} \mid \leq I_{j}^{\max } \quad j=1, \cdots, l \\
& P_{g j}^{\min } \leq P_{g j} \leq P_{g j}^{\max } \quad j=1, \cdots, g_{e d} \\
& Q_{g j}^{\min } \leq Q_{g j} \leq Q_{g j}^{\max } \quad j=1, \cdots, g_{p v} \\
& \Delta u_{j}^{\min } \leq \Delta u_{j} \leq \Delta u_{j}^{\max } \quad j=1, \cdots, r \\
& 0 \leq\left|m_{j}\right| \leq\left|m_{j}\right|^{\max } \quad j=1, \cdots, n,
\end{aligned}
$$

where

$\mu \quad$ penalty coefficient for mismatches

$m_{i} \quad$ mismatch value of the $i^{\text {th }}$ SPQPF equation 
$\lambda_{i}$ weight coefficient of the $j^{\text {th }}$ remedial action

$\Delta u_{j}$ amount of the $j^{\text {th }}$ remedial action adjustment

$n$ number of SPQPF equations

$r$ number of total remedial action control variables

$G(\mathbf{x}, \mathbf{u}, \mathbf{m})=0$ SPQPF equations

$\mathbf{x}$ vector of system state variables

u vector of system remedial action control variables

m vector of mismatch variables

$b \quad$ number of system buses

l number of system circuit branches

$g_{e d}$ number of generators participating economic dispatch

$g_{p v}$ number of PV generators

The first term in the objective function is a penalty function that tends to reduce mismatches to zero and thus reaches feasibility. The second term is a pre-selected function to be optimized. In the present case, as shown in (3.11), this term expresses the total amount of remedial actions. However, it can be substituted with any other functions of interest.

The defined optimization problem is a large-scale problem. The size of this problem can be drastically reduced with simple transformations. Specifically, all mismatch variables can be substituted with one artificial control variable $v$ as follows:

$m_{i}=m_{i}^{0} v, \quad 0 \leq v \leq 1$ 
where variable $v$ represents the normalized change of mismatches. Note that this simple transformation replaces all mismatch variables (a total of $n$ ) with a single variable $v$.

Variables $P_{g j}$ (a total of $g_{e d}$ ) can also be replaced with only two variables that implicitly incorporate the economic dispatch process. Assume that the initial generation $P_{g j}^{0}\left(j=1, \cdots, g_{e d}\right)$ is an economic dispatch schedule with respect to the initial electric load $P_{L}$ and the Lagrange multiplier $\lambda^{0}$. Assume now that the system load has increased from $P_{L}$ to $P_{L}+d P_{L}$ and consider the economic dispatch problem:

$\operatorname{Min} \sum_{j=1}^{g_{e d}} f_{j}\left(P_{g j}\right)=\sum_{j=1}^{g_{e d}}\left(a_{j}+b_{j} P_{g j}+c_{j} P_{g j}^{2}\right)$

S.t. $\quad \sum_{j=1}^{g_{e d}} P_{g j}-\left(P_{L}+d P_{L}\right)-q=0$

where

$q$ system transmission loss.

$a_{j}, b_{j}, c_{j}$ generation cost coefficients for the $j^{\text {th }}$ generating unit.

If $d P_{L}=0$, then the solution for the above problem is $P_{g j}^{0}\left(j=1, \cdots, g_{e d}\right)$. When $d P_{L} \neq 0$, according to the Lagrange theory, the solution must satisfy the following necessary conditions:

$$
\begin{aligned}
& \frac{\partial f_{j}\left(P_{g j}\right)}{\partial P_{g j}}-\lambda\left(1-\frac{d q}{d P_{g j}}\right)=0, \quad j=1, \cdots, g_{e d} \\
& \sum_{j=1}^{g_{e d}} P_{g j}-\left(P_{L}+d P_{L}\right)-q=0 .
\end{aligned}
$$

Upon linearization of the above equations around the point $P_{g j}^{0}$ and $\lambda^{0}$ [52], 
$\left[\begin{array}{ccccc}2 c_{1} & 0 & 0 & \cdots & -\left(1-\frac{d q}{d P_{g 1}}\right) \\ 0 & 2 c_{2} & 0 & \cdots & -\left(1-\frac{d q}{d P_{g 2}}\right) \\ \vdots & & & & \\ \left(1-\frac{d q}{d P_{g 1}}\right) & \left(1-\frac{d q}{d P_{g 2}}\right) & \cdots & 0\end{array}\right]\left[\begin{array}{l}d P_{g 1} \\ \vdots \\ d \lambda\end{array}\right]=\left[\begin{array}{c}0 \\ 0 \\ 0 \\ \vdots \\ d\end{array}\right]$

where $q=\sum_{j=1}^{g_{e d}} P_{g j}-\left(P_{L}+d P_{L}\right)$

and $\frac{d q}{d P_{g j}}=\frac{\partial q}{\partial P_{g j}}-\frac{\partial q}{\partial x} J^{-1} \frac{\partial G}{\partial P_{g j}}$.

The solution of above equations yields:

$$
\begin{aligned}
& {\left[\begin{array}{c}
d P_{g 1} \\
d P_{g 2} \\
d P_{g 3} \\
\vdots \\
d \lambda
\end{array}\right]=\left[\begin{array}{ccccc}
2 c_{1} & 0 & 0 & \cdots & -\left(1-\frac{d q}{d P_{g 1}}\right) \\
0 & 2 c_{2} & 0 & \cdots & -\left(1-\frac{d q}{d P_{g 2}}\right) \\
\vdots & & & & \\
\left(1-\frac{d q}{d P_{g 1}}\right) & \left(1-\frac{d q}{d P_{g 2}}\right) & \cdots & 0
\end{array}\right]^{-1}\left[\begin{array}{l}
0 \\
0 \\
\vdots \\
1
\end{array}\right] d P_{L},} \\
& \text { or } d P_{g j}=p_{f j} d P_{L} \text {, }
\end{aligned}
$$

where $P_{f j}$ is the economic participation factor for generator $j$.

To allow flexibility in the model, two economic participation factors are defined, one $\left(p_{f}^{+}\right)$for a generation increase and the other $\left(p_{f}^{-}\right)$for a generation decrease. Two additional variables $w_{1}$ and $w_{2}$ are introduced to represent the total system generation increase and decrease, respectively. They satisfy the condition $w_{1} w_{2}=0$, which is incorporated into the SPQPF model. Therefore, 
$d P_{g j}=p_{f j}^{+} w_{1}+p_{f j}^{-} w_{2}, \quad j=1, \cdots, g_{e d}$

where

$$
\begin{aligned}
& p_{f j}^{+}=\left\{\begin{aligned}
p_{f j} & P_{g j}^{\min } \leq P_{g j}^{0}<P_{g j}^{\max } \\
0 & P_{g j}^{0}=P_{g j}^{\max }
\end{aligned}\right. \\
& p_{f j}^{-}=\left\{\begin{aligned}
p_{f j} & P_{g j}^{\min }<P_{g j}^{0} \leq P_{g j}^{\max } \\
0 & P_{g j}^{0}=P_{g j}^{\min }
\end{aligned}\right.
\end{aligned}
$$

This transformation guarantees that any changes in unit output will preserve an optimal economic dispatch. Moreover, it reduces the variables of $P_{g j}^{0}\left(j=1, \cdots, g_{e d}\right)$ to only two variables, $w_{1}$ and $w_{2}$.

$$
P_{g j}=P_{g j}^{0}+d P_{g j}=P_{g j}^{0}+p_{f j}^{+} w_{1}+p_{f j}^{-} w_{2}, \quad j=1, \cdots, g_{e d} .
$$

Another feature that has been incorporated in the SPQPF model is the ability to allocate reactive power burden among PV generating units connected to the same bus, which is implemented based on the following derivation:

$$
\begin{aligned}
& \frac{Q_{j i}-Q_{j i}^{\min }}{Q_{j i}^{\max }-Q_{j i}^{\min }}=k_{i}, \quad i=1, \cdots, b_{m p v}, j=1, \cdots, g_{p v i} \\
& Q_{j i}=k_{i}\left(Q_{j i}^{\max }-Q_{j i}^{\min }\right)+Q_{j i}^{\min }, \\
& Q_{i}=\sum_{j=1}^{g_{p v i}} Q_{j i}=k_{i} \sum_{j=1}^{g_{p v i}}\left(Q_{j i}^{\max }-Q_{j i}^{\min }\right)+\sum_{j=1}^{g_{p v i}} Q_{j i}^{\min },
\end{aligned}
$$

where

$k_{i} \quad$ a proportional constant for bus $i$

$b_{m p v}$ number of buses connected to multiple PV generators 
$g_{p v i}$ number of PV generators connected to bus $i$

The substitution of above transformations around the present operating point yields:

Minimize $\quad J=\mu \sum_{i=1}^{n}\left|m_{i}^{0}\right| v+\sum_{j=1}^{r} \lambda_{j}\left|\Delta u_{j}\right|$

Subject to $G^{\prime}\left(\left[\mathbf{x}, \mathbf{k}, w_{1}, w_{2}\right], \mathbf{u}, v\right)=0$

$$
\begin{array}{ll}
V_{j}^{\min } \leq\left|\tilde{V}_{j}\right| \leq V_{j}^{\max } & j=1, \cdots, b \\
\left|\tilde{I}_{j}\right| \leq I_{j}^{\max } & j=1, \cdots, l \\
P_{g j}^{\min } \leq P_{g j} \leq P_{g j}^{\max } & j=1, \cdots, g_{e d} \\
\sum_{j=1}^{g_{p v i}} Q_{j i}^{\min } \leq \sum_{j=1}^{g_{p v i}} Q_{j i} \leq \sum_{j=1}^{g_{p v i}} Q_{j i}^{\max } & i=1, \cdots, b_{m p v}, j=1, \cdots, g_{p v i} \\
Q_{g j}^{\min } \leq Q_{g j} \leq Q_{g j}^{\max } & j=1, \cdots, g_{s p v} \\
\Delta u_{j}^{\min } \leq \Delta u_{j} \leq \Delta u_{j}^{\max } & j=1, \cdots, r \\
0 \leq v \leq 1 & j=1, \cdots, n,
\end{array}
$$

where

$g_{\text {spv }}:$ number of buses with single PV generator.

The solution method for the above problem is iterative. Each iteration includes two steps: (a) the linearization of the objective function and operating constraints and (b) the solution of the resulting linear programming (LP) problem. The efficient co-state method [52] is employed in the first step to compute the sensitivities of the objective function and operating constraints with respect to the control variables. The simplex method can be used in the second step to solve the LP problem. 
During the solution procedure, the mismatch variable $v$ is reduced step by step from one to zero. A nonzero value of mismatch variable $(v \neq 0)$ indicates that the algorithm has not yet converged. In each step, given the allowed minimum mismatch value $v_{\min }$ and participation factors obtained from the solution of the previous step, the power flow problem is solved. Note that in the power flow solution, any generation increase $\left(w_{1} \neq 0\right)$ or decrease $\left(w_{2} \neq 0\right)$ is distributed to individual generating units according to equation (3.18) to preserve economic dispatch. Based on the power flow solution, if any operating constraints are violated, they are added to the set of active constraints in the optimization problem. The solution to the optimization problem provides new remedial actions that force a feasible operating point. Remedial actions may require that one or more generating units be removed from real power economic dispatch and be controlled individually to maintain the feasibility of the operating state. When feasibility has been reinstated, the mismatch variable $v$ is further reduced. This procedure is repeated until the mismatch variable reaches zero. The flow chart of the NDOQPF algorithm is illustrated in Figure 3.7.

The proposed NDOQPF algorithm achieves power flow non-divergence by introducing fictitious sources/mismatches that are driven to zero as the solution progresses. Therefore, if a solution exists, the algorithm guarantees convergence; if a solution does not exist, the algorithm provides a sub-optimal solution that may include load shedding. Also, the solution moves along the economic dispatch for active power and proportional dispatch for reactive power by virtue of Equations (3.10) and (3.13). Note that the algorithm realistically mimics the operating practices of power systems by incorporating remedial actions that alleviate operating constraint violations. Another 
improvement is that violated operating constraints appear gradually since the system is gradually loaded. The appearance of an overwhelming number of violated constraints, which can occur to the traditional power flow solution, is improbable with the proposed algorithm.

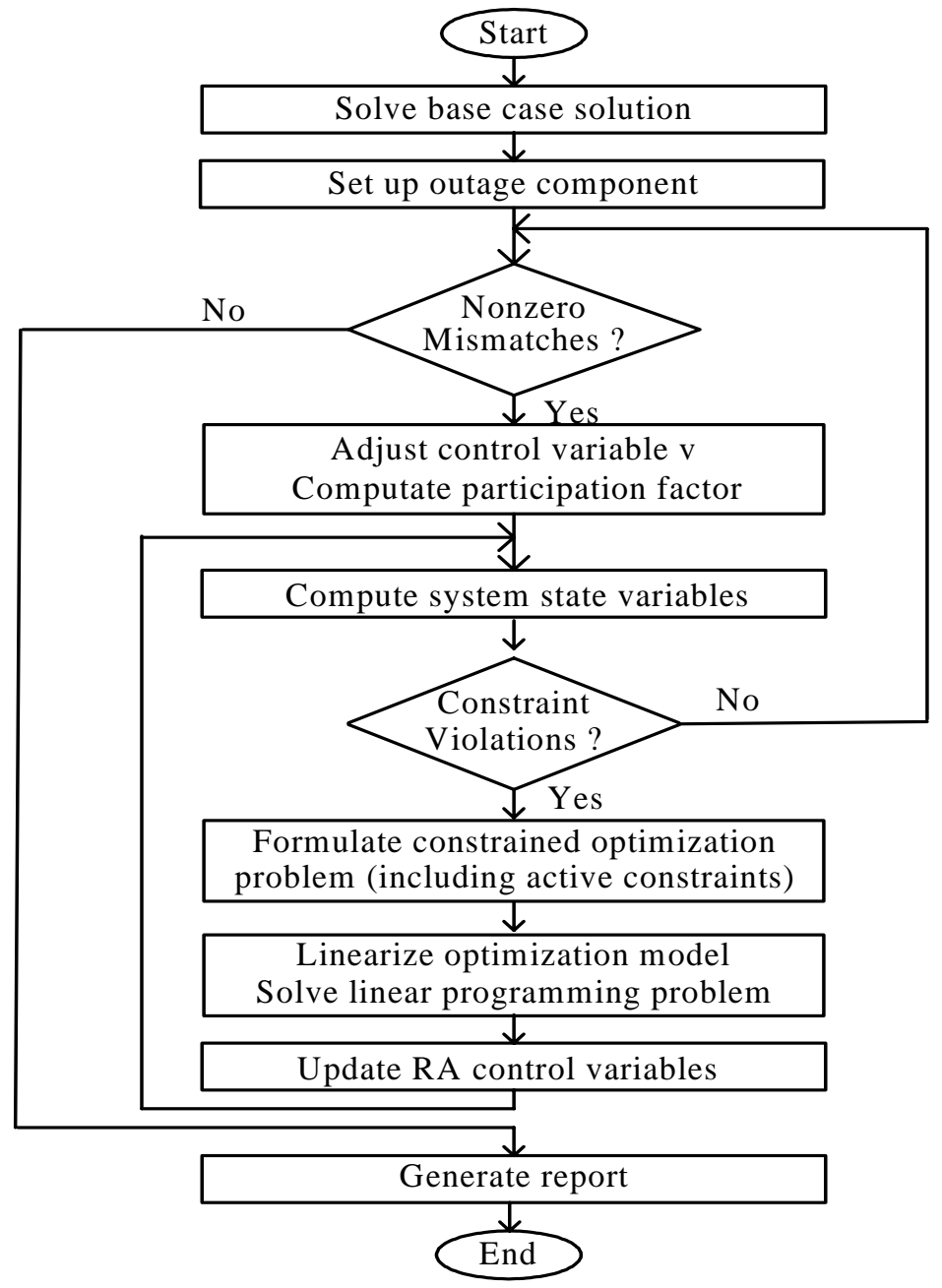

Figure 3.7: Flow chart of the non-divergent optimal quadratized power flow algorithm. 


\subsection{The Application of the NDOQPF Algorithm in Deregulated Power Systems}

The challenge of power system restructuring is to maintain system reliability while creating competitive markets [121]. Many of the current models for the competitive market employ independent transmission operators (ISOs) or regional transmission organizations (RTOs) to operate the transmission system, ensure fair access and system reliability, and provide an open market for power transactions. Many ISOs/RTOs are in operation today in the United States, most notably the PJM RTO, CA ISO, NY ISO, NE ISO, ERCOT ISO, and MISO, and others are currently being established [122-124]. In the power market, energy is the primary commodity and an energy bid is an offer to supply or consume energy (MWhr) at a price (\$) that is submitted by market participants (power suppliers or consumers). Market participants offer their bids to the ISO/RTO operator that collects and maintains competitive offers for each trading interval. Upon receiving these bids, the ISO/RTO uses this information to determine the least cost market / schedule of generation while satisfying customer demand and system reliability requirements. If transmission congestion occurs, the power bids, which may include possible remedial actions to relieve congestion, are appropriately selected. This process, referred to as congestion management, is no different from typical remedial action procedures used in the regulated environment except that it allows power bids as one of remedial actions.

Mathematically, this ISO/RTO operational procedure is formulated as a constrained optimization problem. The objective is to minimize the overall system cost (defined with energy bids) while satisfying system constraints. This objective function is usually 
piecewise linear or staircase. The constraints of the optimization problem are power flow equations, transmission capability limits, bus voltage limits, and so on. Specifically, this constrained optimization problem is formulated as follows:

Minimize $\quad J=\sum_{i=1}^{n} C_{i}\left(P_{g i}\right)$

Subject to $G(x, u)=0$

$$
\begin{array}{cc}
V_{i}^{\min } \leq\left|\tilde{V}_{i}\right| \leq V_{i}^{\max } & i=1, \cdots, b \\
\left|\tilde{T}_{i}(x, u)\right| \leq \bar{T}_{i} & i=1, \cdots, l \\
P_{g i}^{\min } \leq P_{g i} \leq P_{g i}^{\max } & i=1, \cdots, n \\
Q_{g i}^{\min } \leq Q_{g i} \leq Q_{g i}^{\max } & i=1, \cdots, g \\
u_{i}^{\min } \leq u_{i} \leq u_{i}^{\max } & i=1, \cdots, r,
\end{array}
$$

where

$C_{i}$ bidding function of the generator $i$

$n$ number of generators participating in the market

$G(x, u)=0$ power flow equations

$x$ vector of system state variables

$u$ vector of remedial action control variables

$b$ number of system buses

$\tilde{T}_{i}$ load of transmission line $i$

$\bar{T}_{i}$ load limit of transmission line $i$

l number of circuit branches

$P_{g i}$ real power output of generator $i$ 
$Q_{g i}$ reactive power output of generator $i$

$g$ number of total generators

$r$ number of total control variables

This problem is formulated as a NDOQPF. Note that congestion management is incorporated in the optimization problem by introducing remedial action control vector $u$. The formulated constrained optimization problem in the deregulated environment is no different from that in the regulated deregulated environment. Therefore, the proposed NDOQPF algorithm is capable of efficiently solving the generic ISO/RTO operational model. In fact, the NDOQPF algorithm is particularly effective in the deregulated environment. Due to the impact of deregulation on the system operation, the transmission system is more likely to be heavily stressed, as it may be operated in various or unusual power flow patterns from what it was originally designed for [125-126]. Because of these new scenarios appearing in the deregulated environment, power flow analysis under contingencies will inevitably encounter divergent situations more frequently than it will in regulated power systems. The NDOQPF algorithm can efficiently solve such power flow non-convergent problems and provide always a solution (optimal or suboptimal) to the ISO/RTO operator. Indeed, its ability to always provide a solution is a major advantage of the proposed methodology. 


\subsection{The Reliability Index Computation}

Once the contingency effects analysis is complete, a set of system states (contingencies) that satisfy the pre-defined system failure criterion is identified from the system state space. The probabilities of system states in this set and the transition rates from any state inside the set to a state outside the set are used to calculate reliability indices. The reliability index calculation is illustrated by the example system state space shown in Figure 3.8.

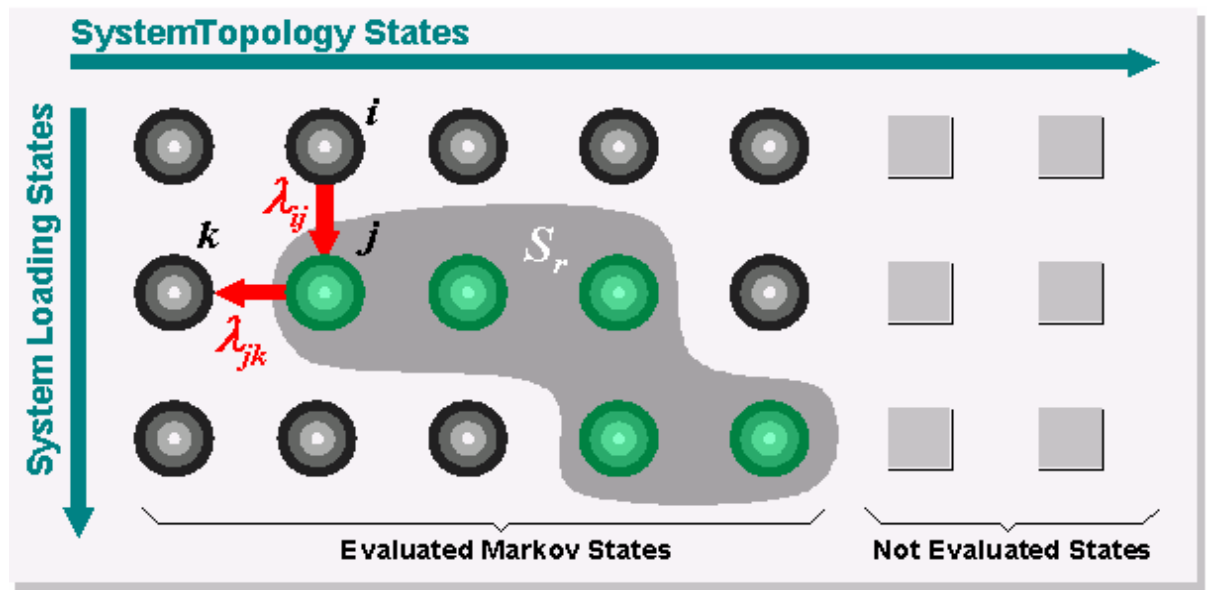

Figure 3.8: An example system state space diagram.

This system state space includes states that have been evaluated and not evaluated. They are distinguished by the critical contingency selection and the effects analysis. Among the evaluated states, system state $j$ at a certain load level is characterized by its occurrence probability $p_{j}$ and associated transition rates to and from other system states, such as $\lambda_{j k}$ and $\lambda_{i j}$. The probability $p_{j}$ of system state $j$ can be calculated based on the 
probability of every independent component working status (up or down) in this state by the following direct method:

$$
p_{j}=\prod_{i=1}^{n} p_{c i},
$$

where

$p_{j}$ probability of system state $j$

$p_{c i}$ probability of independent component $i$ in its own working status

$n$ number of independent components in system state $j$.

A set of system states $\left(S_{r}\right)$ that contains the evaluated states that possess some common features such as a system failure is identified by retrieving the stored results of the effects analysis. Based on the identified set $S_{r}$ as well as the probability and transition rates associated with each system state (contingency) in this set, three different classes of reliability indices can be computed: (a) the probability index, (b) the frequency index, and (c) the duration index.

\section{Probability Index}

The probability of $S_{r}, P_{r}\left[S_{r}\right]$, is obtained by adding all the probabilities $p_{j}$ :

$P_{r}\left[S_{r}\right]=\sum_{j \in S_{r}} p_{j}$.

The probabilities $p_{j}, j \in S_{r}$, can be added because the events of being in any of the states $j$ are mutually exclusive. 


\section{Frequency Index}

The frequency of $S_{r}, f_{S_{r}}$, is the sum of the frequency of leaving a state $j$ inside $S_{r}$ for a state $i$ outside $S_{r}$, which can be calculated as follows:

$$
f_{S_{r}}=\sum_{i \notin S_{r}} \sum_{j \in S_{r}} f_{j i}=\sum_{i \notin S_{r}} \sum_{j \in S_{r}} p_{j} \lambda_{j i}=\sum_{j \in S_{r}}\left(p_{j} \sum_{i \notin S_{r}} \lambda_{j i}\right)
$$

where

$\lambda_{j i} \quad$ transition rate from state $j$ to state $i$

$f_{j i}$ frequency of transferring from state $j$ to state $i$, which is defined as the expected number of direct transfers from $j$ to $i$ per unit time. The relationship between $f_{j i}$ and $\lambda_{j i}$ can be written as $f_{j i}=\lambda_{j i} p_{i}$.

\section{Duration Index}

The duration index of $S_{r}, T_{S_{r}}$, is the ratio of the probability index over the frequency index:

$T_{S_{r}}=\frac{P_{r}\left[S_{r}\right]}{f_{S_{r}}}$.

These reliability indices indicate the system reliability level quantitatively, which can help system planners and operators to plan and operate power systems with an acceptable reliability level. 


\subsection{Summary}

Based on the quadratized power flow model, a comprehensive approach for bulk power system reliability assessment is proposed in this chapter. In this approach, an improved contingency selection technique is developed to perform critical contingency selection and ranking with high accuracy, and a non-divergent optimal quadratized power flow algorithm is implemented to conduct effects analysis realistically and efficiently. In addition, reliability index computation is also included in this approach. In the next chapter, a method to evaluate the effects of protection system hidden failures on bulk power system reliability is put forward, and the proposed framework in this chapter will be extended to evaluate also contingencies resulting from protection system hidden failures. 


\section{CHAPTER IV \\ EFFECTS OF PROTECTION SYSTEM HIDDEN FAILURES ON BULK POWER SYSTEM RELIABILITY}

\subsection{Introduction}

Current bulk power system reliability assessment practice mainly focuses on the analysis of $\mathrm{N}-1$ contingencies and some credible $\mathrm{N}-2$ cases. The component outage modes involved in these contingencies generally include independent and common-mode outages. Most contingencies that involve multiple component outages are considered the result of several independent, successive events in the system, and they are usually not a major concern in the reliability assessment procedure. However, recent research [128-130] shows that protection system hidden failures may cause multiple component outages that are dependent upon each other, i.e., an initial component outage event can lead to the cascading tripping of other intact components because of a malfunction in the protection

system. Consequently, protection system hidden failures have been recognized as a contributing factor in spreading power system disturbances and even causing system blackouts. Since protection systems are generally assumed to be perfect when bulk power system reliability is considered, the effects of protection system hidden failures have not been taken into account in the current practice of bulk power system reliability assessment. 
This chapter will discuss protection system hidden failures. First, it will provide some background information, including a definition, characteristics, and examples. Then, for the purpose of considering protection system hidden failures in the reliability assessment procedure, it will propose a breaker-oriented substation model composed of detailed substation configurations and protection system schemes in the system network model. In addition, it will introduce advanced system real time monitoring and analysis technologies brought by the application of electronic intelligent devices in modern substations. The proposed approach assesses the impact of such technologies on detecting protection system hidden failures. Then this chapter will present the development of a hidden failure effects analysis method that can identify hidden failure outages following any possible initial equipment outages in each system substation. Finally, by expanding the security-constrained adequacy evaluation methodology proposed in the previous section, this chapter will evaluate contingencies resulting from hidden failure outages and include the effects of protection system hidden failures on bulk power system reliability.

\subsection{Protection System Hidden Failures}

A hidden failure in the protection system is defined in [17] as "a permanent defect that will cause a relay or a relay system to incorrectly and inappropriately remove a circuit element(s) as a direct consequence of another switching event.” In other words, hidden failures remain hidden during normal system conditions, and when system disturbances occur, such as faults or overloads occur, hidden failures are exposed and cause 
unnecessary outages of intact equipment. The existence of hidden failures in protection systems exacerbates an already stressed system situation and reduces the system reliability level.

Protection systems consist of many components, such as transducers (current and voltage transformers), relays, and circuit breakers, which contribute to the detection and removal of faults [127]. Each of these protection system components may suffer from hidden failures depending on the inherent mechanism of the component. Some example hidden failures that may exist in these major protection system components are briefly analyzed below.

\section{Current Transformer (CT)}

After a fault occurs, fault currents may cause the current transformer core to become saturated, in which the secondary current of the current transformer cannot faithfully represent the primary current.

\section{Voltage Transformer (VT)}

For some voltage transformers, such as coupling capacitor voltage transformers (CCVTs), when a fault occurs and causes the system voltage to decrease from the normal value to a lower value, the output of the voltage transformer may be significantly different from the actual primary voltage during the transient procedure.

\section{Relay}

When the system operating condition changes, if the setting of an influenced relay does not change accordingly, the relay may fail to detect the system status correctly under 
some system disturbances and then operate inappropriately as a result of the outdated relay setting.

\section{Circuit Breaker}

Any failures in the trip mechanism of a circuit breaker, such as the open circuit of trip coils and the failure to separate circuit breaker plates because of welding or obstacles in the plate motion paths, will cause the circuit breaker fail to trip when it is required to open the faulty circuit.

Most of present research focuses on the study of hidden failures in relays. For example, the mechanism and consequence of some possible hidden failure modes in various relays used for transmission system protections are analyzed in [131, 132]. On the other hand, the analysis of hidden failures in other protection system components, such as transducers and circuit breakers, has not received much attention.

\subsection{The Breaker-Oriented Substation Model}

Since protection systems are assumed to be perfect in the current bulk power system reliability assessment procedure, system substations are generally simplified to buses, and different transmission lines simply converge at buses to connect generators or to serve loads. In this study, to consider the effects of protection system hidden failures on bulk power system reliability, we convert each bus in the general power system model into a breaker-oriented substation with a specific bus arrangement (breaker and a half, ring, and 
so on) $[133,134]$. The selection of bus arrangements follows usual design procedures and practices. Breaker-oriented substation models become an integral part of the system network model and reflect the real life existence of substation configurations. Figure 4.1 illustrates a breaker-oriented substation model that consists of a breaker-and-a-half bus arrangement, six circuit breakers (CB1 to CB6), and four incoming/outgoing transmission lines (L1 to L4).

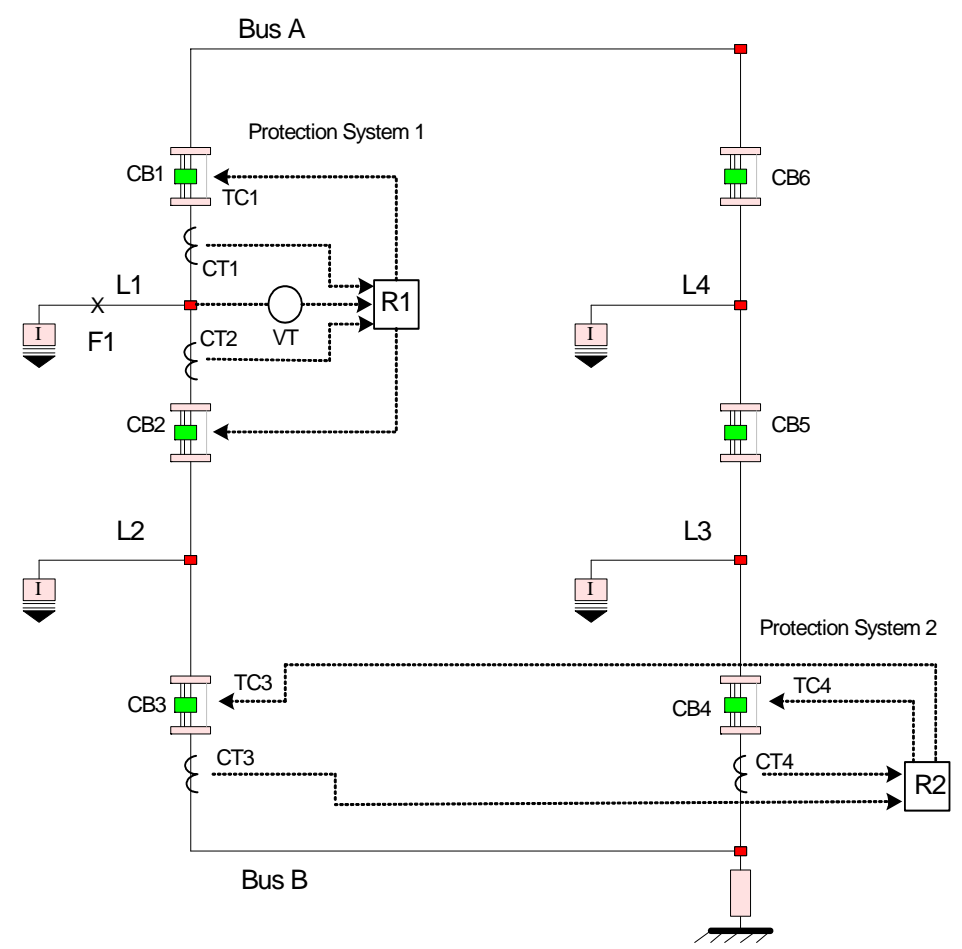

Figure 4.1: A breaker-and-a-half bus arrangement substation model.

The incorporation of breaker-oriented substation models adds a new level of detail in the system network model, based on which the protection system schemes for various power system components can be introduced into the network model as well. For instance, two examples of protection system schemes are presented in Figure 4.1. The purpose of protection systems 1 and 2 is to protect transmission line L1 and Bus B, 
respectively. The major components in these two protection systems include current transformers (CT1 to CT4), a voltage transformer (VT), relays (R1 and R2), and circuit breakers (CB1 to CB4) associated with trip coils (TC1 to TC4). The detailed substation and protection system models make the study of the impact of protection system performance on power system operation feasible. Specifically, in this work, the effects of protection system hidden failures on bulk power system reliability are investigated.

\subsection{The Impact of Advanced System Monitoring and Analysis Technologies}

Nowadays, besides traditional conventional remote terminal units (RTUs), a variety of intelligent electronic devices (IEDs), such as phasor measurement units (PMUs) and digital protection relays, have become available in modern power system substations. Compared to the limitation, inaccuracy, and delay of the traditional system substation data obtained from RTUs, more redundant, accurate, and real time system data can be obtained from IEDs. Based on the measurements of IEDs as well as RTUs, the substation level system information extraction process, including substation level state estimation and alarm processing, can significantly advance the capability of system real time monitoring [135-137].

The advanced system monitoring and analysis functions brought by the application of IEDs can detect protection system hidden failures existing in transducers and relays considerably. In particular, real time validation and verification can be performed for transducer outputs and relay settings. If any hidden failures exist in current or voltage 
transformers that cause the outputs of these transducers to fail to reflect actual system status, the substation level state estimation can identify bad data in a rapid, reliable way. In addition, based on the real time synchronized measurements of system states obtained from PMUs, the real time system model can be built and relay settings can be verified on line to avoid any protection system malfunction caused by outdated relay settings. However, such advanced monitoring and analysis technologies cannot detect the trip abilities of circuit breakers. Hidden failures in the circuit breaker trip mechanism such as the welding plates of circuit breakers will remain uncovered until circuit breakers fail to open during system disturbances. Hence, in this work, the consideration of protection system hidden failures concentrates on hidden failures in the circuit breaker trip mechanism.

\subsection{Probabilistic Modeling of Hidden Failures in the Circuit Breaker Trip Mechanism}

This section presents the probabilistic modeling of hidden failures in the circuit breaker trip mechanism (CBTM). For the example substation model in Figure 4.1, which has six circuit breakers, the independent and common-mode hidden failure models of CBTMs are described below.

\section{Independent Hidden Failures of CBTMs}

Each CBTM can cycle between the normal and the hidden failure status. This process can be modeled as a two-state Markov process with constant transition rates. We assume 
that the occurrences of such hidden failures are independent with their own failure and repair rates. Two-state Markov models for the six CBTMs (CBTM1 to CBTM6) are shown in Figure 4.2, in which $\lambda_{x}$ and $\mu_{x}$ represent the failure and repair rates of each CBTM, respectively.

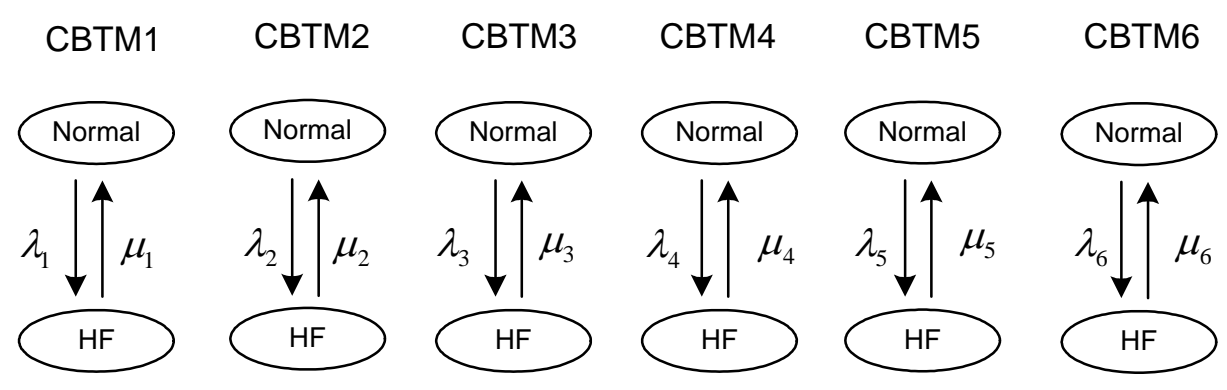

Figure 4.2: Two-state Markov models of CBTMs.

The differential equations that govern transitions for each CBTM between the normal and hidden failure status are given as follows:

$\frac{d \mathbf{p}_{X}(t)}{d t}=\mathbf{p}_{X}(t) \mathbf{A}_{X}$,

where $\mathbf{p}_{X}(t)$ is the row vector that contains normal and hidden failure status probabilities (i.e., $p_{X}(t)$ and $\left.q_{X}(t)\right)$ of each $\operatorname{CBTM} X$, $\mathbf{p}_{X}(t)=\left[p_{X}(t) q_{X}(t)\right]$.

Also, the probabilities of the normal and the hidden failure status for each CBTM satisfy the following condition:

$$
p_{X}(t)+q_{X}(t)=1,
$$

where $0 \leq p_{X}(t) \leq 1$ and $0 \leq q_{X}(t) \leq 1$. In addition, $\mathbf{A}_{X}$ is the transition intensity matrix for CBTM $X$, i.e.,

$$
\mathbf{A}_{X}=\left[\begin{array}{rr}
-\lambda_{X} & \lambda_{X} \\
\mu_{X} & -\mu_{X}
\end{array}\right] .
$$


The initial condition represents a CBTM with the probability of normal status set to one and the probability of hidden failure status set to zero.

$\mathbf{p}_{X}(0)=[1 \quad 0]$

The solution to the above differential equations gives the probabilities of the normal and the hidden failure status of each CBTM $X$ :

$$
\begin{aligned}
& p_{X}(t)=\frac{\mu_{X}}{\lambda_{X}+\mu_{X}}+\frac{\lambda_{X}}{\lambda_{X}+\mu_{X}} \exp \left(-\left(\lambda_{X}+\mu_{X}\right) t\right) \\
& q_{X}(t)=1-p_{X}(t)=\frac{\lambda_{X}}{\lambda_{X}+\mu_{X}}-\frac{\lambda_{X}}{\lambda_{X}+\mu_{X}} \exp \left(-\left(\lambda_{X}+\mu_{X}\right) t\right) .
\end{aligned}
$$

If only long-term status probabilities are of interest, the normal and hidden failure status probabilities of each CBTM are expressed as follows:

$$
p_{X}(\infty)=\frac{\mu_{X}}{\lambda_{X}+\mu_{X}}, \quad q_{X}(\infty)=\frac{\lambda_{X}}{\lambda_{X}+\mu_{X}} .
$$

For the substation shown in Figure 4.1, each combination of the operational status of each of the six CBTMs constitutes a substation state. The different combinations of CBTM statuses generate a total of $64\left(2^{6}\right)$ substation states in the state space. Part of this state space (states 1 to 16) is shown in Table 4.1. 
Table 4.1: State enumeration for the example substation (states 1-16).

\begin{tabular}{|c|c|c|c|c|c|c|c|c|c|c|c|c|c|c|c|c|}
\hline$\#$ & 1 & 2 & 3 & 4 & 5 & 6 & 7 & 8 & 9 & 10 & 11 & 12 & 13 & 14 & 15 & 16 \\
\hline CВTM1 & & $\mathrm{X}$ & & & & & & $\mathrm{X}$ & $\mathrm{X}$ & $\mathrm{X}$ & $\mathrm{X}$ & $\mathrm{X}$ & & & & \\
\hline CВTM2 & & $\mathrm{X}$ & & & & & $\mathrm{X}$ & & & & & $\mathrm{X}$ & $\mathrm{X}$ & $\mathrm{X}$ & $\mathrm{X}$ \\
\hline CBTM3 & & & $\mathrm{X}$ & & & & & $\mathrm{X}$ & & & & $\mathrm{X}$ & & & \\
\hline CBTM4 & & & & $\mathrm{X}$ & & & & & $\mathrm{X}$ & & & & $\mathrm{X}$ & & \\
\hline CTMB5 & & & & & $\mathrm{X}$ & & & & & $\mathrm{X}$ & & & & $\mathrm{X}$ & \\
\hline CВTM6 & & & & & & $\mathrm{X}$ & & & & & $\mathrm{X}$ & & & & $\mathrm{X}$ \\
\hline
\end{tabular}

$\mathrm{X}$ indicates a hidden failure status of the CBTM

Because we assume that hidden failures of CBTMs are independent, the probability of each substation state can be obtained by multiplying the probability of each CBTM status. For example, for substation state 3 in Table 4.1, in which CBTM2 is in hidden failure status and all others are in normal status, the probability for this substation state is expressed as follows:

$$
p_{S 3}(t)=p_{C B T M 1}(t) q_{C B T M 2}(t) \prod_{i=3}^{6} p_{C B T M i}(t)
$$

The sum of the substation state probabilities is forced to 1 simply because the total 64 states are mutually exclusive and their union forms the certain event, i.e. ,

$$
\sum_{k=1}^{64} p_{S k}(t)=1
$$

\section{Common-Mode Hidden Failures of CBTMs}

The independent hidden failure model of CBTMs does not account for common-mode failures, which involve simultaneous hidden failures of two or more CBTMs as a result of a single event. For example, a loss of the power resource that supplies power to two or 
more trip coils can cause multiple circuit breakers to enter hidden failure status simultaneously. When considering common-mode hidden failures of the CBTM in the substation, shown in Figure 4.1, the differential equations that govern the transitions among substation states, initial conditions, and the sum of probabilities are given below:

$$
\begin{aligned}
& \frac{d \mathbf{p}_{S}(t)}{d t}=\mathbf{p}_{S}(t) \mathbf{A}_{S}, \\
& \mathbf{p}_{S}(0)=\left[\begin{array}{lllll}
1 & 0 & 0 & \cdots & 0
\end{array}\right], \\
& \sum_{k=1}^{64} p_{S k}(t)=1,
\end{aligned}
$$

where $\mathbf{p}_{S}(t)$ is the row vector of substation state probabilities, and $\mathbf{A}_{S}$ is the substation transition intensity matrix. Off-diagonal terms $(i, j)$ of matrix $\mathbf{A}_{S}$ have the failure/repair rate from state $i$ to state $j$, and diagonal terms are the negative sum of the failure and repair rates of all transitions from the current state to an adjacent state (transition rates found in the same row). The sum of all the elements in each row of the transition matrix is zero. The initial state is assumed to be at state 1 in Table 4.1, in which every component works in the normal status. The solution to the differential equations gives the probability of each substation state at any instant in time.

\subsection{Effects Analysis of CBTM Hidden Failures}

Hidden failures in CBTMs can cause the trip of intact equipment following system disturbances, which reduce the level of system reliability. In this section, a hidden failure 
effects analysis method is proposed to identify additional outages caused by such hidden failures given initial faults. This method is illustrated by using the example substation shown in Figure 4.1.

We assume that the substation is operating under state 3 as enumerated in Table 4.1, in which the trip mechanism of circuit breaker 2 (CBTM2) has a hidden failure that can cause circuit breaker 2 (CB2) to fail to open while all other circuit breakers are in the normal operating status. If an initial fault F1 occurs in transmission line L1, circuit breakers 1 and 2 should open to isolate the faulty circuit L1 accordingly. Since CB2 fails to open because of its hidden failure, circuit breaker 3 (CB3) that is adjacent to CB2 will open and result in the outage of the intact component of transmission line L2 following the initial fault on transmission line L1. The conditional probability of the hidden failure outage of transmission line L2, given the incidence of the initial fault on L1, is the product of the occurrence probability of substation state 3 and the probabilities of all other substations in their specific states, expressed as follows:

$$
P_{c d_{-} s 3}(t)=p_{S 3}(t) \prod_{i=1}^{N} p_{i k}(t),
$$

where

$p_{S 3}(t)$ probability of the example substation in state 3

$p_{i k}(t)$ probability of the substation $i$ in state $k$

$N \quad$ total number of the rest substations in the system

Such an effects analysis procedure can be repeated for all other possible initial faults. The results, including initial faulty circuits, associated hidden failure outages, and corresponding conditional probabilities are listed in Table 4.2. We can see that the initial 
faults on L1 or L2 will cause outages of intact equipment in the substation under state 3, while the initial faults on other components do not cause any hidden failure outages. For the example substation with a total of 64 substation states, a cut-off probability can be predefined to reduce states in the state space that are subject to hidden failure effects analysis. The states with probabilities less than the cut-off probability will not be considered because of their small incidence. Furthermore, the hidden failure effects analysis procedure can be performed for all substation states and all substations in the system.

After all hidden failure outages following each initial system disturbance are obtained through the above hidden failure effects analysis procedure, the proposed framework of bulk power system reliability assessment, i.e., the security-constrained adequacy evaluation (SCAE) methodology, is extended to include these hidden failure outages. Specifically, the SCAE methodology evaluates system contingencies resulting from independent outages, common-mode outages, and hidden failure outages. All these contingencies are subject to the three main steps in SCAE: (a) critical contingency selection, (b) effects analysis, and (c) reliability index computation. In such a way, the effects of protection system hidden failures on bulk power system reliability are included in the bulk power system reliability assessment procedure. 
Table 4.2: Hidden failure effects analysis for substation state 3.

\begin{tabular}{|c|c|c|}
\hline Initial Fault & Hidden Failure Outage & Conditional Probability \\
\hline Fault on Bus A & N/A & N/A \\
\hline Fault on L1 & L2 & $p_{s 3}$ \\
\hline Fault on L2 & L1 & $p_{s 3}$ \\
\hline Fault on Bus B & N/A & N/A \\
\hline Fault on L3 & N/A & N/A \\
\hline Fault on L4 & N/A & N/A \\
\hline
\end{tabular}

\subsection{Summary}

A method for evaluating the effects of protection system hidden failures on bulk power system reliability is developed in this chapter. In this method, by replacing each system bus with a substation configuration, the breaker-oriented system network model is developed to take into account protection system performance on system reliability. Also, the influence of advanced system real-time monitoring and analysis technologies on the detection of protection system hidden failures is analyzed. Based on such analysis results, this work focuses on identifying contingencies resulting from the hidden failure in the circuit breaker trip mechanism. The framework for bulk power system reliability assessment proposed in the previous chapter is then extended to include contingencies resulting from CBTM hidden failures such that the effects of protection system hidden failures on system reliability is included in the reliability assessment procedure. In the next chapter, the demonstration and application of the proposed comprehensive approach for bulk power system reliability assessment are provided. 


\section{CHAPTER V}

\section{APPROACH DEMONSTRATION AND APPLICATION}

\subsection{Approach Demonstration}

In this section, the IEEE 24-bus reliability test system and its derivation, i.e., the circuit breaker-oriented 24-substation reliability test system, are used to demonstrate three advanced techniques developed to implement the proposed comprehensive approach for bulk power system reliability assessment: (1) the system state linearization approach for the critical contingency selection, (2) the non-divergent optimal quadratized power flow algorithm for the contingency effects analysis, and (3) the hidden failure effects analysis method for evaluating the impact of protection system hidden failures on bulk power system reliability.

The IEEE 24-bus reliability test system [138], as shown in Figure 5.1, was developed by the IEEE reliability subcommittee and published in 1979 as a benchmark for testing various reliability analysis methods. In this test system, the generation system contains 32 generating units, ranging from $12 \mathrm{MW}$ to $400 \mathrm{MW}$, and the transmission system contains 24 load or generation buses connected by 38 transmission lines or transformers at two voltage levels, $138 \mathrm{kV}$ and $230 \mathrm{kV}$. This test system is used to test the system state linearization approach and the non-divergent optimal quadratized power flow algorithm. In addition, the hidden failure effects analysis method for evaluating the impact of protection system hidden failures on bulk power system reliability is demonstrated with 
the circuit breaker-oriented 24-substation reliability test system, shown in Figure 5.2 in section 5.1.3. The circuit breaker-oriented 24-substation reliability test system is derived from the original IEEE 24-bus reliability test system by converting each bus in the original IEEE 24-bus reliability test system to a substation with a specific bus arrangement.

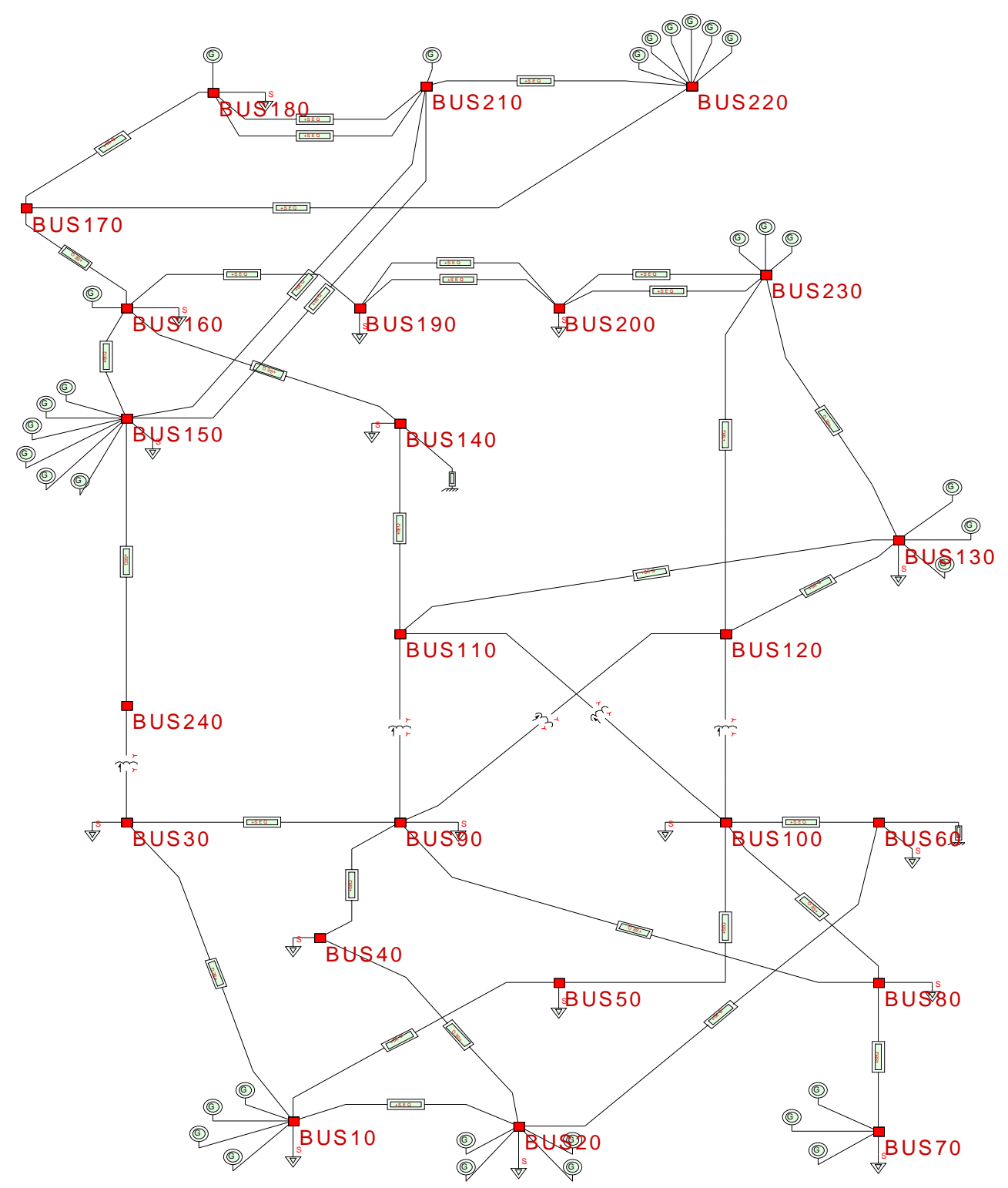

Figure 5.1: The IEEE 24-bus reliability test system. 
In both reliability test systems, the system peak load level is applied, and available remedial actions include generating unit real power re-dispatching, generating unit and the synchronous condenser reactive power rescheduling, reactor bank switching, and load shedding. The representative results illustrate the features of these advanced techniques.

\subsubsection{System State Linearization Approach}

To demonstrate the effectiveness of the proposed system state linearization approach for critical contingency selection and ranking, the first-level contingencies of the IEEE 24-bus reliability test system are ranked using three different contingency selection and ranking methods in terms of two types of performance index changes: (1) the currentbased circuit loading index and (2) the voltage index, as defined in section 3.3.1. The three contingency selection and ranking methods include (1) the full power flow solution method, (2) the traditional performance index linearization method, and (3) the proposed system state linearization method. The full power flow solution method calculates the exact performance index changes resulting from contingencies and provides a complete accurate ranking of the contingencies. The contingency ranking results obtained from the full power flow solution method can be used as the standard to verify the contingency selection and ranking accuracy of the other two methods. The second method, the traditional performance index linearization method, predicts the linear approximation of the exact performance index change by taking into account only the first-order term of the Taylor's series in calculating the performance index change before and after a contingency. The third method, the proposed system state linearization method, can 
predict the nonlinearity of performance index changes to a certain extent by including higher-order terms in Taylor's series.

Based on these three contingency selection and ranking methods, changes in the two types of performance indices are computed for the first-level contingencies resulting from independent transmission circuit outages, and these contingencies are ranked according to performance index changes. Parts of these results are provided in Tables 5.1 and 5.2. Table 5.1 shows the changes in the current-based circuit loading index and subsequent contingency ranking results of the three methods. Table 5.2 shows the changes in the voltage index and subsequent contingency ranking results of the three methods. Such results demonstrate that the proposed system state linearization method can predict performance index changes more accurately than the traditional performance index linearization method because the indirect differentiation procedure of the system state linearization method yields higher-order sensitivity terms when calculating performance index changes. Consequently, the system state linearization method can result in less misranking in critical contingency selection and ranking than the traditional performance index linearization technique, which results in severe misranking as shown in Table 5.1.

For the first-level contingencies resulting from independent generating unit outages, the three methods are applied to calculate the current-based circuit loading index changes and subsequent rank contingencies. Part of such performance index changes and contingency ranking results shown in Table 5.3 reveals that the traditional performance index linearization method has produced misranking, while the proposed system state linearization method provides complete, accurate contingency ranking. These results indicate that generating unit outages cause low nonlinearity in performance index 
variations, and the traditional performance index linearization method can present satisfactory ranking results. Hence, the ranking of contingencies resulting from generating unit outages is generally not a major concern in critical contingency selection and ranking.

Table 5.1: Changes in the current-based circuit loading index and contingency ranking results for independent transmission circuit outages.

\begin{tabular}{|c|c|c|c|c|c|c|}
\hline $\begin{array}{l}\text { Outage } \\
\text { Circuit }\end{array}$ & $\begin{array}{c}\text { Exact } \\
\Delta J\end{array}$ & $\begin{array}{c}\text { Exact } \\
\text { Ranking }\end{array}$ & $\begin{array}{c}\text { Traditional PI } \\
\text { Linearization } \\
\Delta J\end{array}$ & $\begin{array}{c}\text { Traditional PI } \\
\text { Linearization } \\
\text { Ranking }\end{array}$ & $\begin{array}{c}\text { Proposed } \\
\text { System State } \\
\text { Lnearization } \\
\Delta J\end{array}$ & $\begin{array}{c}\text { Proposed } \\
\text { System State } \\
\text { Linearization } \\
\text { Ranking }\end{array}$ \\
\hline C60-100 & 60.5755 & 1 & -3.1615 & 33 & 4.16212 & 1 \\
\hline C150-240 & 2.91412 & 2 & 0.03554 & 14 & 1.08256 & 5 \\
\hline С30-240 & 2.91410 & 3 & 0.05060 & 10 & 2.11779 & 4 \\
\hline C140-160 & 2.5400 & 4 & -0.28261 & 31 & 3.30945 & 2 \\
\hline C160-170 & 1.4861 & 5 & -0.34598 & 32 & 2.51369 & 3 \\
\hline C20-60 & 1.3223 & 6 & 0.42555 & 1 & 0.63109 & 9 \\
\hline C200-230 & 1.1885 & 7 & 0.15394 & 2 & 0.90109 & 6 \\
\hline C120-230 & 1.1719 & 8 & 0.12725 & 4 & 0.88311 & 7 \\
\hline C160-190 & 0.9607 & 9 & 0.03769 & 13 & 0.21585 & 13 \\
\hline C150-210 & 0.8729 & 10 & 0.15079 & 3 & 0.64768 & 8 \\
\hline
\end{tabular}

(C: CIRCUIT) 
Table 5.2: Changes in the voltage index and contingency ranking results for independent transmission circuit outages.

\begin{tabular}{|l|c|c|c|c|c|c|}
\hline \multicolumn{1}{|c|}{$\begin{array}{c}\text { Outage } \\
\text { Circuit }\end{array}$} & $\begin{array}{c}\text { Exact } \\
\Delta J\end{array}$ & $\begin{array}{c}\text { Exact } \\
\text { Ranking }\end{array}$ & $\begin{array}{c}\text { Traditional PI } \\
\text { Linearization } \\
\Delta J\end{array}$ & $\begin{array}{c}\text { Traditional PI } \\
\text { Linearization } \\
\text { Ranking }\end{array}$ & $\begin{array}{c}\text { Proposed } \\
\text { System State } \\
\text { Linearization } \\
\Delta J\end{array}$ & $\begin{array}{c}\text { Proposed } \\
\text { System State } \\
\text { Linearization } \\
\text { Ranking }\end{array}$ \\
\hline C60-100 & $29,578.0$ & 1 & -0.86953 & 33 & 36.17562 & 1 \\
\hline C150-240 & 14.6924 & 2 & 0.19610 & 2 & 0.91132 & 3 \\
\hline C30-240 & 4.92840 & 3 & 0.03831 & 7 & 0.32333 & 7 \\
\hline C20-40 & 1.2586 & 4 & -0.00177 & 19 & 0.06838 & 17 \\
\hline C140-160 & 0.9363 & 5 & 0.03405 & 8 & 0.84005 & 4 \\
\hline C160-170 & 0.7549 & 6 & 0.25152 & 1 & 0.60161 & 5 \\
\hline C10-30 & 0.4042 & 7 & 0.07364 & 3 & 0.21500 & 8 \\
\hline C90-120 & 0.31384 & 8 & 0.05423 & 5 & 0.10594 & 11 \\
\hline C160-190 & 0.3014 & 9 & 0.07017 & 4 & 0.11739 & 10 \\
\hline C90-110 & 0.16475 & 10 & 0.03251 & 9 & 0.05693 & 19 \\
\hline
\end{tabular}

(C: CIRCUIT) 
Table 5.3: Changes in the current-based circuit loading index and contingency ranking results for independent generating unit outages.

\begin{tabular}{|l|c|c|c|c|c|c|}
\hline \multicolumn{1}{|c|}{$\begin{array}{c}\text { Outage } \\
\text { Unit }\end{array}$} & $\begin{array}{c}\text { Exact } \\
\Delta J\end{array}$ & $\begin{array}{c}\text { Exact } \\
\text { Ranking }\end{array}$ & $\begin{array}{c}\text { Traditional PI } \\
\text { Linearization } \\
\Delta J\end{array}$ & $\begin{array}{c}\text { Traditional PI } \\
\text { Linearization } \\
\text { Ranking }\end{array}$ & $\begin{array}{c}\text { Proposed } \\
\text { System State } \\
\text { Linearization } \\
\Delta J\end{array}$ & $\begin{array}{c}\text { Proposed } \\
\text { System State } \\
\text { Linearization } \\
\text { Ranking }\end{array}$ \\
\hline G20 - 3,4 & 0.42871 & 1 & 0.28979 & 1 & 0.42353 & 1 \\
\hline G10 - 3,4 & 0.36657 & 2 & 0.25198 & 2 & 0.36217 & 2 \\
\hline G20 - 1,2 & 0.0403 & 3 & 0.03813 & 3 & 0.04025 & 3 \\
\hline G10 - 1,2 & 0.03512 & 4 & 0.03316 & 4 & 0.03508 & 4 \\
\hline G150 -1 5 & -0.04601 & 5 & -0.04726 & 5 & -0.04589 & 5 \\
\hline G70 -1 3 & -0.129 & 6 & -0.84040 & 11 & -0.12283 & 6 \\
\hline G230 -1,2 & -0.25074 & 7 & -0.34508 & 7 & -0.24495 & 7 \\
\hline G220 -1 6 & -0.27395 & 8 & -0.30632 & 6 & -0.27054 & 8 \\
\hline G160 & -0.3335 & 9 & -0.49543 & 8 & -0.32745 & 9 \\
\hline G230 - 3 & -0.36234 & 10 & -0.77921 & 10 & -0.33188 & 10 \\
\hline
\end{tabular}

(G: GENERATOR)

\subsubsection{Non-Divergent Optimal Quadratized Power Flow Algorithm}

Based on the results of contingency selection and ranking, the non-divergent optimal quadratized power flow (NDOQPF) algorithm is applied to the most critical contingencies for their effects analysis. Part of the contingency effects analysis results for first-level contingencies resulting from both independent and common-mode transmission circuit outages is listed in Table 5.4. For each contingency, the result of the effects analysis shows if it causes any operating constraint violations; when constraint violations occur, the result also shows if load shedding is required to maintain normal 
system operation; whenever load shedding is necessary, the corresponding contingency is recorded as a system failure state and make a non-zero contribution to system unreliability. Table 5.4 shows that six contingencies that result from independent outages and four contingencies that result from common-mode outages cause constraint violations and require load shedding for the system to operate normally. They represent system failure states. Some of the remaining contingencies may cause constraint violations, but remedial actions without load shedding can maintain normal system operation. Such evaluation results reflect the ability of the NDOQPF algorithm to simulate contingencies in a realistic manner to capture the system response including all major controls and adjustments.

Table 5.4: Part of the effects analysis results of first-level contingencies.

\begin{tabular}{|c|c|c|c|c|c|}
\hline No. & Outage Mode & $\begin{array}{c}\text { Outage } \\
\text { Components }\end{array}$ & $\begin{array}{c}\text { Constraints } \\
\text { Violations } \\
\text { (Yes/No })\end{array}$ & $\begin{array}{c}\text { RAs w/o Load } \\
\text { Shedding } \\
\text { (Yes/No) }\end{array}$ & $\begin{array}{c}\text { Load } \\
\text { Shedding } \\
\text { (Yes/No) }\end{array}$ \\
\hline 1 & Independent & C60-100 & Yes & Yes & Yes \\
\hline 2 & Independent & C150-240 & Yes & Yes & Yes \\
\hline 3 & Independent & C30-240 & Yes & Yes & Yes \\
\hline 4 & Independent & C160-170 & Yes & Yes & Yes \\
\hline 5 & Independent & C20-60 & Yes & Yes & Yes \\
\hline 6 & Independent & C120-230 & Yes & Yes & Yes \\
\hline 7 & Common-mode & C70-80 & Yes & Yes & Yes \\
\hline 8 & Common-mode & C190-200 & Yes & Yes & Yes \\
\hline 9 & Common-mode & C200-230 & Yes & Yes & Yes \\
\hline 10 & Common-mode & C150-210 & Yes & Yes & Yes \\
\hline
\end{tabular}

(C: CiRCUIT G: GENERATING UNIT) 
Regarding the second-level or higher-level contingencies, using the traditional power flow algorithm such as the Newton-Raphson method to conduct the effects analysis may produce non-convergence because of the severe impact of high-level contingencies on system operation. This non-convergence problem can be solved using the NDOQPF algorithm. For example, consider the second-level contingency that involves the outages of two circuits C30-90 (first level) and C150-240 (second level). The traditional power flow under this second-level contingency diverges, while the NDOQPF algorithm converges and provides a list of remedial actions. For illustrative purposes, the effects analysis procedure and results of this contingency are detailed below.

The first-level outage of circuit C30-90 does not cause any constraint violations when control variable $v$ is reduced from 1.0 directly to 0.0 . The solution of the first-level outage, represented with $\mathbf{x}^{0}$ (the vector of system state variables) and $\mathbf{u}^{0}$ (the vector of system control variables), is used as the base case for the second-level outage of C150240. Under the operating point $\left(\mathbf{x}^{0}, \mathbf{u}^{0}\right)$, after the first-level outage and given the additional outage of circuit C150-240, the mismatch vector $\mathbf{m}^{0}$ is calculated first. The artificial control variable $v$ is then gradually reduced as follows:

$v: 1.0 \rightarrow 0.667 \rightarrow 0.333 \rightarrow 0.0$.

Note that when $v$ is 1.0 , the mismatch vector is $\mathbf{m}^{0}$, and the operating point is $\mathbf{x}^{0}$ and $\mathbf{u}^{0}$, the system does not have any constraint violations. In the progress of the solution, the optimization problem shown in Equation 3.22 is formulated at each step of the control variable $v$ if constraint violations exist and solved using the linear programming technique. The procedure is repeated until variable $v$ reaches zero. During the solution procedure, the voltages at bus 30 and bus 240 are lower than their lower 
limits when each time variable $v$ is reduced to a new level. Therefore, major active constraints in the formulated optimization problem include the following two inequality constraints:

$V_{30} \geq V_{30}^{\min }$,

$V_{240} \geq V_{240}^{\min }$.

Such violated constraints cannot be eliminated completely by the available remedial actions (excluding load shedding). Therefore, to maintain the normal system operating conditions, load shedding is applied as a remedial action, and $63 \%$ of the system load at bus 30 is tripped.

Load $_{\text {shedding_bus } 30}=113.82 M W+j 23.4 M V A R$.

Corresponding to this load change, the reduction in the total amounts of system real power and reactive power outputs are as follows:

$\Delta P=106.91 M W, \Delta Q=23.4 M W$.

The solution of this second-level contingency illustrates the major advantage of the NDOQPF algorithm in achieving the non-divergent solution when the system is overstressed by multi-level contingencies.

\subsubsection{Protection System Hidden Failure Effects Analysis}

The effects of protection system hidden failures on bulk power system reliability are evaluated using a circuit breaker-oriented, 24-substation reliability test system as shown in Figure 5.2. The circuit breaker-oriented, 24-substation reliability system is mostly 
derived from the original IEEE 24-bus reliability test system. The original IEEE 24-bus reliability test system is a bus-oriented system, and the approach used to develop the circuit breaker-oriented system is to replace each node (bus) of the original system with a substation that has specific bus arrangement (e.g., ring, breaker and a half, and so on). The bus arrangement at each node and the location of each circuit breaker become the explicit part of the network model.

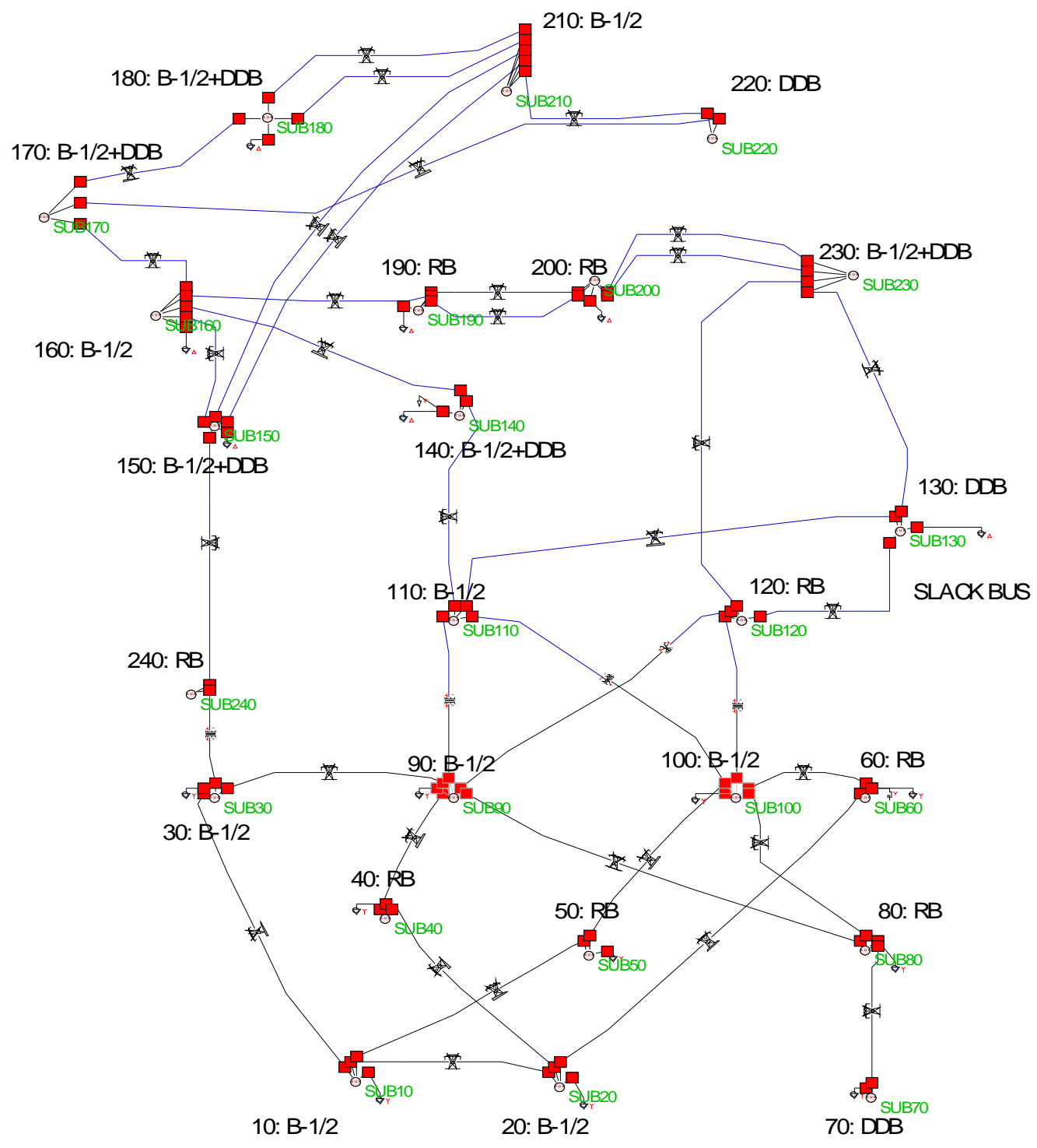

Figure 5.2: A circuit breaker-oriented, 24-substation reliability test system. 
As an example, bus 180 of the original IEEE 24-bus reliability test system, which connects to one unit, three circuits, and one system load, is replaced by substation 180 with a mixed breaker-and-a-half and a double-breaker scheme as illustrated in Figure 5.3. The overall conversion procedure from the original bus-oriented system to the breakeroriented system amounts to replacing each bus of the original IEEE 24-bus reliability test system with a substation. To make the overall model more realistic, we have selected a variety of bus arrangements, such as breaker-and-a-half, double-breaker, ring bus, and so on. Therefore, the proposed circuit breaker-oriented 24-substation reliability test system includes substations of various breaker arrangements and different reliability levels. A summary of substation topology is provided in Table 5.5 .

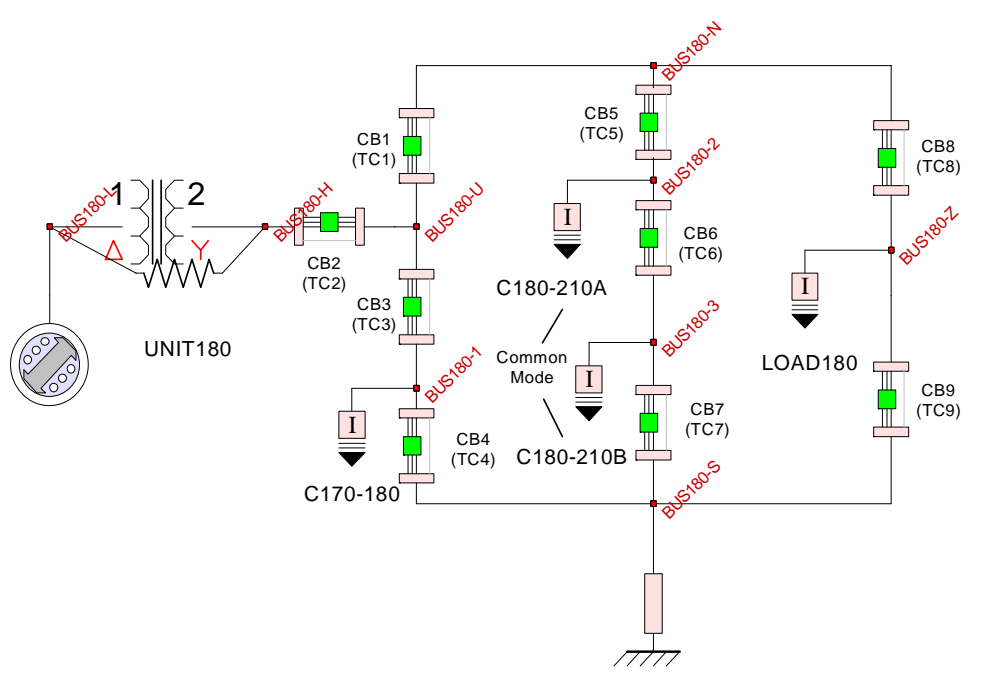

Figure 5.3: A circuit breaker-oriented model of substation 180. 
Table 5.5: Substation topology summary of the circuit breaker-oriented 24-substation reliability test system.

\begin{tabular}{|l|l|}
\hline Bus Arrangement & Substation Number \\
\hline Double-breaker & $70,130,220,240$ \\
\hline Breaker-and-a-half & $10,20,30,90,100,110,160,210$ \\
\hline $\begin{array}{l}\text { Mixed double-breaker } \\
\text { and breaker-and-a-half }\end{array}$ & $140,150,170,180,230$ \\
\hline Ring-bus & $40,50,60,80,120,190,200$ \\
\hline
\end{tabular}

Based on the circuit breaker-oriented system model, the effects analysis of the circuit breaker trip mechanism (CBTM) hidden failure is performed for each substation. We consider two different levels of CBTM hidden failure probabilities to illustrate the impact of different CBTM hidden failure probability levels on bulk power system reliability. Note that only independent hidden failure outages are concerned in the CBTM hidden failure effects analysis. Under these assumptions, contingencies resulting from hidden failure outages in substation 180 are listed in Table 5.6. The results include the initial faulty circuits and the corresponding hidden failure outages.

After the contingencies resulting from CBTM hidden failures for all substations are obtained and consolidated, the security-constrained adequacy evaluation approach is applied to evaluate contingencies resulting from independent, common-mode, and hidden failure outages. The reliability evaluation result of first-level contingencies resulting from transmission circuit outages shows that six contingencies that result from independent outages, four contingencies that result from the common-mode outages, and forty-seven contingencies that result from hidden failure outages lead to system unreliability. These 
contingencies are provided in Table 5.7, in which only a portion of the contingencies resulting from hidden failure outages are listed. The table shows that most of contingencies that lead to system failures are contingencies resulting from hidden failure outages because of intact system component outages following initial system faults, which exacerbate an already stressed system.

The reliability indices of probability, frequency, and duration of such system loss-ofload events are calculated for both situations with and without the consideration of contingencies resulting from hidden failure outages. In situations in which contingencies resulting from hidden failure outages are included, the hidden failure probabilities of circuit breakers are assumed at a level of 10e-2. All these results are shown in Table 5.8, which indicate that hidden failures in protection systems can downgrade the system reliability level. 
Table 5.6: Summary of the effects analysis for substation 180 .

\begin{tabular}{|l|l|}
\hline Initial Faulty Circuit & Hidden Failure Outage \\
\hline Fault on BUS180-U & C170-180 \\
\hline Fault on C170-180 or BUS180-1 & UNIT180 \\
\hline Fault on C180-210A or BUS180-2 & C180-210B \\
\hline Fault on C180-210B or BUS180-3 & C180-210A \\
\hline Fault on BUS180-N & UNIT180 \\
\cline { 2 - 2 } & C180-210A \\
\cline { 2 - 2 } & LOAD180 \\
\hline Fault on BUS180-S & C170-180 \\
\cline { 2 - 2 } & C180-210B \\
\cline { 2 - 2 } & LOAD180 \\
\hline Fault on UNIT180 & N/A \\
\hline \multirow{2}{*}{$\begin{array}{l}\text { Fault on LOAD180 or BUS180-Z } \\
\text { Common-mode outage of }\end{array}$} & N10A and C180-210B \\
\hline
\end{tabular}


Table 5.7: List of contingencies leading to system unreliability.

\begin{tabular}{|c|c|c|}
\hline Contingency Type & $\begin{array}{l}\text { Contingency } \\
\text { Number }\end{array}$ & Outage Component \\
\hline \multirow{6}{*}{ Independent outages } & 1 & C60-100 \\
\hline & 2 & C150-240 \\
\hline & 3 & C30-240 \\
\hline & 4 & C160-170 \\
\hline & 5 & C20-60 \\
\hline & 6 & C120-230 \\
\hline \multirow{4}{*}{$\begin{array}{c}\text { Common-mode } \\
\text { outages }\end{array}$} & 7 & C70-80, G70-1,2,3 \\
\hline & 8 & C190-200 \\
\hline & 9 & C200-230 \\
\hline & 10 & C150-210 \\
\hline \multirow{10}{*}{$\begin{array}{l}\text { Hidden failure } \\
\text { outages }\end{array}$} & 11 & C30-90, C30-240 \\
\hline & 12 & C20-40,C40-90 \\
\hline & 13 & C50-100,C10-50 \\
\hline & 14 & C60-100,C20-60 \\
\hline & 15 & C80-100,C80-90 \\
\hline & 16 & C60-100,C50-100 \\
\hline & 17 & C110-140,C140-160 \\
\hline & 18 & C160-190,C160-170 \\
\hline & 19 & C150-210, C150-240 \\
\hline & 20 & C30-240,C150-240 \\
\hline
\end{tabular}


Table 5.8: Comparison of reliability indices with and without contingencies resulting from hidden failure outages.

\begin{tabular}{|c|c|c|}
\hline $\begin{array}{c}\text { Reliability } \\
\text { Index }\end{array}$ & $\begin{array}{c}\text { W/o Contingencies } \\
\text { Resulting from Hidden } \\
\text { Failure Outages }\end{array}$ & $\begin{array}{c}\text { With Contingencies } \\
\text { Resulting from Hidden } \\
\text { Failure Outages }\end{array}$ \\
\hline Probability & $6.976 \mathrm{e}-4$ & $8.903 \mathrm{e}-4$ \\
\hline Frequency (/yr) & 0.261 & 0.384 \\
\hline Duration (hrs) & 23.428 & 20.320 \\
\hline
\end{tabular}

\subsection{Approach Application}

In this section, the proposed comprehensive approach for bulk power system reliability assessment is applied to evaluate the reliability of two circuit breaker-oriented reliability test systems: (1) the circuit breaker-oriented, 24-substation reliability test system shown in Figure 5.2 and (2) the circuit breaker-oriented, 73-substation reliability test system derived from an enhanced IEEE reliability test system [139]. The enhanced IEEE reliability test system, developed by the Reliability Test System Task Force of the Application of Probability Methods Subcommittee in 1996, is an extension version of the original IEEE 24-bus reliability test system. It is a multi-area reliability test system that links three single IEEE 24-bus reliability test systems, shown in Figure 5.4. In this multiarea system, areas A and B are connected through three interconnections: (1) a $230 \mathrm{kV}$ transmission line connecting bus 123 and bus 217, (2) a 230kV transmission line connecting bus 113 and bus 215, and (3) a 138kV transmission line connecting bus 107 and bus 203. Area C is connected to areas A and B through two interconnections: (1) a 
$230 \mathrm{kV}$ transmission line connecting area B at bus 223 to area C at bus 318 and (2) a $230 \mathrm{kV}$ transmission line connecting area $\mathrm{A}$ at bus 121 to area $\mathrm{C}$ at bus 325 . In addition, a transformer has been added between bus 325 and bus 323 in area C. In this enhanced IEEE reliability test system, the generation system contains 96 generating units, ranging from 12MW to $400 \mathrm{MW}$, and the transmission system contains 73 load or generation buses connected by 120 transmission lines or transformers at two voltage levels, $138 \mathrm{kV}$ and $230 \mathrm{kV}$. The conversion of this IEEE 73-bus reliability test system to the circuit breaker-oriented, 73-substation reliability test system is done by converting each bus in the IEEE 73-bus reliability test system to a substation with a specific bus arrangement. A summary of the substation topology is provided in Table 5.9.

Table 5.9: The substation topology summary of the circuit breaker-oriented, 73susbstation reliability test system.

\begin{tabular}{|c|c|}
\hline Bus Arrangement & Substation No. \\
\hline Double-breaker & $\begin{array}{l}\text { 107, 113,122, 124, 207, 213, 222, 224, } \\
307,313,322,324,325\end{array}$ \\
\hline Breaker-and-a-half & $\begin{array}{l}\text { 101, 102, 103, 109, 110, 111, 116, 121, } \\
\text { 201, 202, 203, 209, 210, 211, 216, 221, } \\
301,302,303,309,310,311,316,321\end{array}$ \\
\hline $\begin{array}{l}\text { Mixed double-breaker } \\
\text { and breaker-and-a-half }\end{array}$ & $\begin{array}{l}\text { 114, 115, 117, 118, 123, 214, 215, 217, } \\
218,223,314,315,317,318,323\end{array}$ \\
\hline Ring-bus & $\begin{array}{l}\text { 104, 105, 106, 108, 112, 119, 120, 204, } \\
\text { 205, 206, 208, 212, 219, 220, 304, 305, } \\
\text { 306, 308, 312, 319, 320 }\end{array}$ \\
\hline
\end{tabular}

The proposed comprehensive approach for bulk power system reliability assessment is applied to evaluate the reliability of two circuit breaker-oriented reliability test systems shown in Figures 5.2 and 5.4. Reliability indices are calculated under different component outage modes, component outage levels, and load levels for the two systems. 


\section{Component Outage Modes}

The proposed bulk power system reliability assessment approach consists of three types of component outage modes: (1) independent outages, (2) common-mode outages, and (3) hidden failure outages. Independent outages are generally the basic outage mode considered in the reliability analysis, based on which reliability indices are calculated for the following three situations: (1) consider only independent outages, (2) consider both independent and common-mode outages, and (3) consider all the three outage modes.

\section{Outage Levels of Transmission Circuits and Generating Units}

The outage levels of transmission circuits and generating units refer to the maximum numbers of transmission circuit or generating unit outage events allowed in a contingency. For example, if both the transmission circuit outage level and the generating unit outage level are selected as one, contingencies with up to one transmission circuit outage event and one generating unit outage event will be considered in the reliability analysis procedure. All possible combinations of outage events that should be considered in a contingency under this case are enumerated in Table 5.10. Note that the simultaneous failures of two or more components in a common-mode outage or a hidden failure outage account for only one outage event.

Table 5.10: Enumeration of outage event combinations given the transmission circuit and generating unit outage levels as one.

\begin{tabular}{|l|c|c|c|c|}
\hline \multicolumn{1}{|c|}{ Combination List } & 1 & 2 & 3 & 4 \\
\hline $\begin{array}{l}\text { Number of transmission circuit } \\
\text { outage event }\end{array}$ & 0 & 1 & 0 & 1 \\
\hline $\begin{array}{l}\text { Number of generating } \\
\text { unit outage event }\end{array}$ & 0 & 0 & 1 & 1 \\
\hline
\end{tabular}




\section{Load Levels}

To assess bulk power system reliability under different system load levels, the system load model at bus $k$ is defined as follows:

$P_{k L}=a_{k 0}+a_{k 1} v_{1}+a_{k 2} v_{2}$

where

$a_{k 0}, a_{k 1}$, and $a_{k 2}$ real power load control coefficients at bus $k$

$v_{1}$ and $v_{2} \quad$ independent system load level control variables

The real power load control coefficient $a_{k 0}$ is the real power load value at bus $k$ in the base case, and coefficients $a_{k 1}$ and $a_{k 2}$ can be positive or negative real power load values for each load in the system. The number of discrete values that independent load level control variables $v_{1}$ and $v_{2}$ can take determines how many load levels will be considered in the reliability analysis, and each specific combination of $v_{1}$ and $v_{2}$ determines an actual load level. Some examples of the number of discrete values that load level control variables $v_{1}$ and $v_{2}$ can take and the corresponding load levels resulting from different combinations of such values are listed in Table 5.11. 
Table 5.11: The number of discrete values of independent load level control variables and corresponding load levels

\begin{tabular}{|c|c|c|}
\hline $\begin{array}{l}\text { Number of Discrete Values of } \\
\text { Independent Load Level } \\
\text { Control Variables } v_{1} \text { and } v_{2}\end{array}$ & Values of $v_{1}$ and $v_{2}$ & Corresponding Load Levels \\
\hline 1 & $\begin{array}{l}v_{1}=1 \\
v_{2}=1\end{array}$ & $v_{1}=1, v_{2}=1$ \\
\hline 2 & $\begin{array}{l}v_{1}=(0,1) \\
v_{2}=(0,1)\end{array}$ & $\begin{array}{l}v_{1}=1, v_{2}=1 \\
v_{1}=0, v_{2}=0 \\
v_{1}=1, v_{2}=0 \\
v_{1}=0, v_{2}=1\end{array}$ \\
\hline
\end{tabular}

The reliability indices for the two circuit breaker-oriented reliability test systems under different component outage modes, component outage levels, and load levels are provided in Tables $5.12 \sim 5.17$. Three load levels used in the reliability assessment are provided in Table 5.18. Based on these results, we can draw the following conclusions:

(1) The impact of hidden failure outages on the level of system reliability is significant. Figures $5.5 \sim 5.8$ illustrate the variation of probability and frequency indices when considering hidden failure outages.

(2) The level of system reliability decreases with the increases of the system load level and the component outage level. Figures $5.5 \sim 5.10$ illustrate the variation of probability and frequency indices when considering different load levels and component outage levels.

(3) The influence of generating unit outages on the level of system reliability is more substantial than that of transmission circuit outages because generating units have much higher failure probabilities and longer reparation time than transmission circuits. The 
contributions to the probability index from transmission circuit outages and generating unit outages under the independent outage mode and the low load level of the 24substation system, which are obtained from Tables 5.12 and 5.15, are shown in Figure 5.11.

(4) The level of system reliability increases with the increase of the system scale, which is illustrated by the comparison of probability and frequency indices of two reliability test systems under different component outage modes and load levels, shown in Figures 5.12 $\sim 5.15$. 


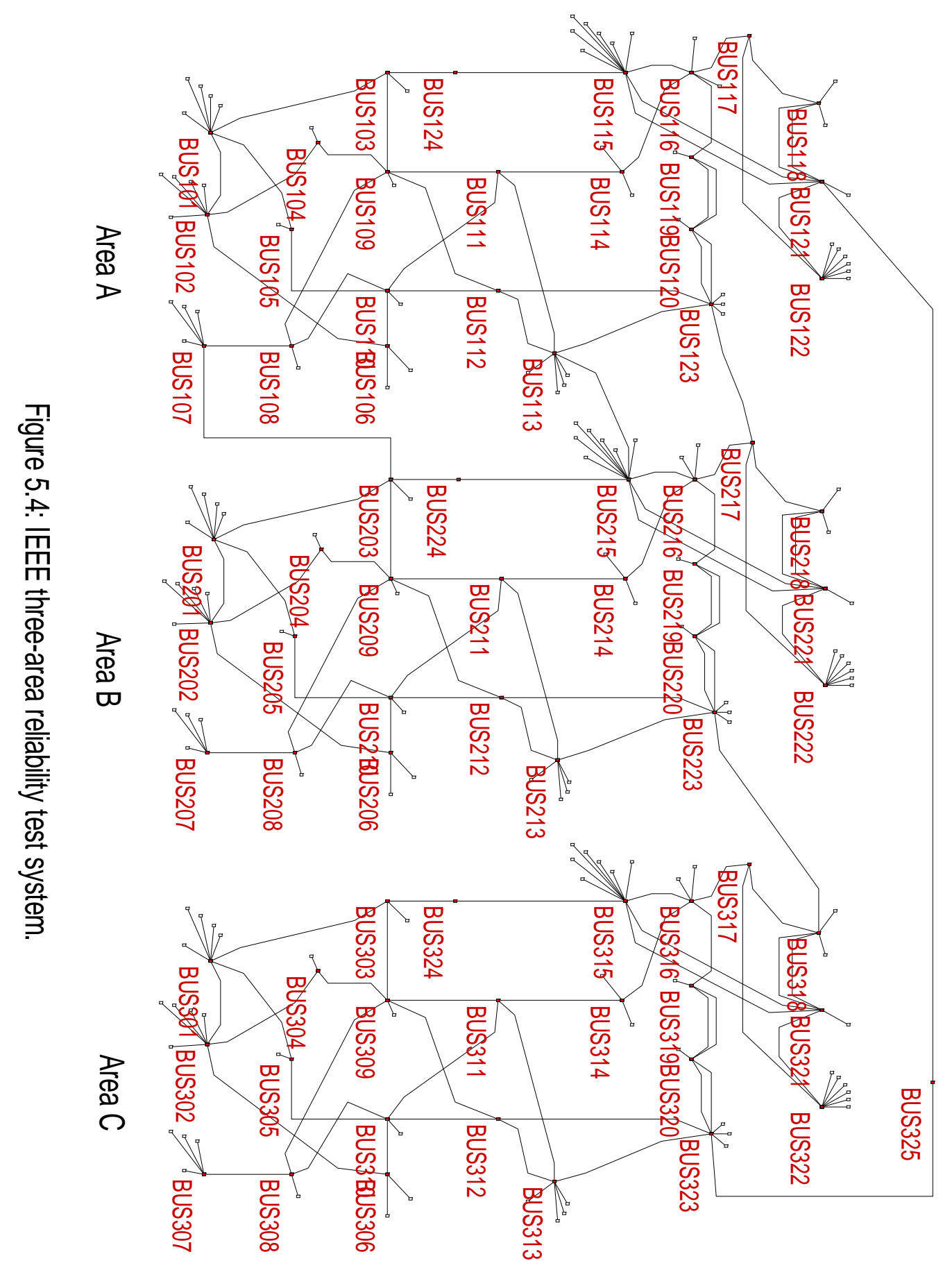


Table 5.12: The reliability assessment of the 24-substation reliability test system (circuit outage level: 1 , generating unit outage level: 0 ).

\begin{tabular}{|c|l|c|c|c|}
\hline \multirow{4}{*}{$\begin{array}{c}\text { Load } \\
\text { Level }\end{array}$} & \multicolumn{1}{|c|}{$\begin{array}{c}\text { Reliability } \\
\text { Index }\end{array}$} & Independent & $\begin{array}{c}\text { Ondependent and } \\
\text { Common-mode }\end{array}$ & $\begin{array}{c}\text { Independent, Common- } \\
\text { mode, and Hidden } \\
\text { Failure }\end{array}$ \\
\cline { 3 - 5 } & Probability & $6.9761 \mathrm{e}-4$ & $6.977 \mathrm{e}-4$ & $8.903 \mathrm{e}-4$ \\
\hline \multirow{4}{*}{ Peak } & Frequency (/yr) & 0.2608 & 0.2609 & 0.3838 \\
\cline { 2 - 6 } & Duration (hrs) & 23.4279 & 23.4242 & 20.3202 \\
\hline \multirow{4}{*}{ Low } & Probability & $6.3309 \mathrm{e}-4$ & $6.3312 \mathrm{e}-4$ & $8.0457 \mathrm{e}-4$ \\
\cline { 2 - 6 } & Frequency (/yr) & 0.2043 & 0.204348 & 0.3109 \\
\cline { 2 - 6 } & Duration (hrs) & 27.1404 & 27.1404 & 22.6675 \\
\cline { 2 - 5 } & Probability & $3.0438 \mathrm{e}-4$ & $3.0438 \mathrm{e}-4$ & $4.7234 \mathrm{e}-4$ \\
\cline { 2 - 5 } & Frequency (/yr) & 0.1420 & 0.1420 & 0.2361 \\
\cline { 2 - 5 } & Duration (hrs) & 18.7754 & 18.7754 & 17.5229 \\
\hline
\end{tabular}
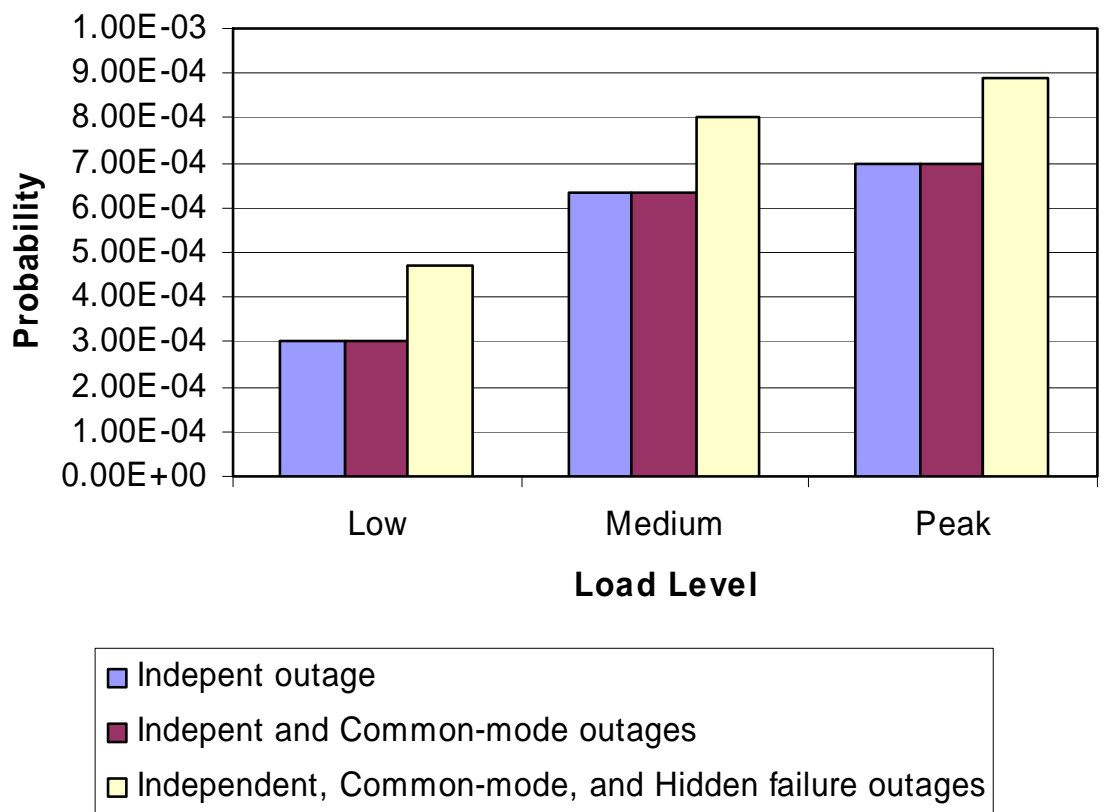

Figure 5.5: The probability indices of the 24-substation reliability test system under different component outage modes and load levels (circuit outage level: 1, generating unit outage level: 0 ). 


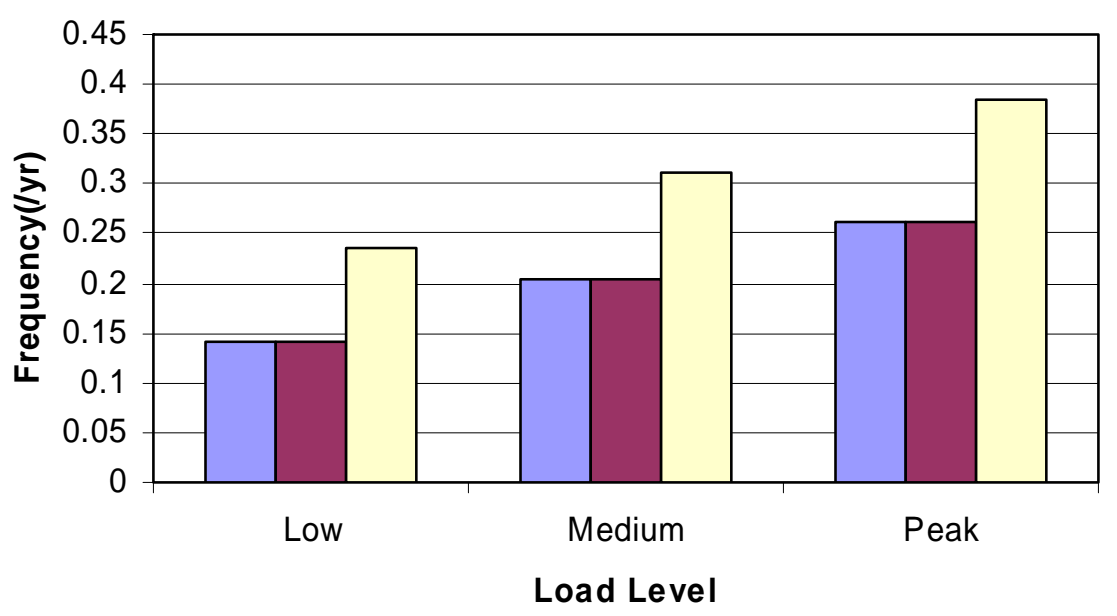

Indepent outage

Indepent and Common-mode outages

$\square$ Independent, Common-mode, and Hidden failure outages

Figure 5.6: The frequency indices of the 24-substation reliability test system under different component outage modes and load levels

(circuit outage level: 1, generating unit outage level: 0 ).

Table 5.13: The reliability assessment of the 24-substation reliability test system (circuit outage level: 2, generating unit outage level: 0 ).

\begin{tabular}{|c|l|c|c|c|}
\hline \multirow{4}{*}{$\begin{array}{c}\text { Load } \\
\text { Level }\end{array}$} & \multicolumn{1}{|c|}{$\begin{array}{c}\text { Reliability } \\
\text { Index }\end{array}$} & Independent & $\begin{array}{c}\text { Independent and } \\
\text { Common-mode }\end{array}$ & $\begin{array}{c}\text { Independent, Common- } \\
\text { mode, and Hidden } \\
\text { Failure }\end{array}$ \\
\cline { 3 - 5 } & Probability & $7.1549 \mathrm{e}-4$ & $7.1549 \mathrm{e}-4$ & $9.1493 \mathrm{e}-4$ \\
\cline { 2 - 5 } & Frequency (/yr) & 0.2681 & 0.2681 & 0.3944 \\
\cline { 2 - 6 } & Duration (hrs) & 23.3799 & 23.3866 & 20.3203 \\
\hline \multirow{4}{*}{ Medium } & Probability & $6.4844 \mathrm{e}-4$ & $6.4844 \mathrm{e}-4$ & $8.2603 \mathrm{e}-4$ \\
\cline { 2 - 6 } & Frequency (/yr) & 0.2094 & 0.2094 & 0.3191 \\
\cline { 2 - 5 } & Duration (hrs) & 27.1229 & 27.1255 & 22.6778 \\
\hline \multirow{3}{*}{ Low } & Probability & $3.1677 \mathrm{e}-4$ & $3.1673 \mathrm{e}-4$ & $4.9070 \mathrm{e}-4$ \\
\cline { 2 - 5 } & Frequency (/yr) & 0.1504 & 0.1503 & 0.2479 \\
\cline { 2 - 5 } & Duration (hrs) & 18.4559 & 18.4611 & 17.3420 \\
\hline
\end{tabular}




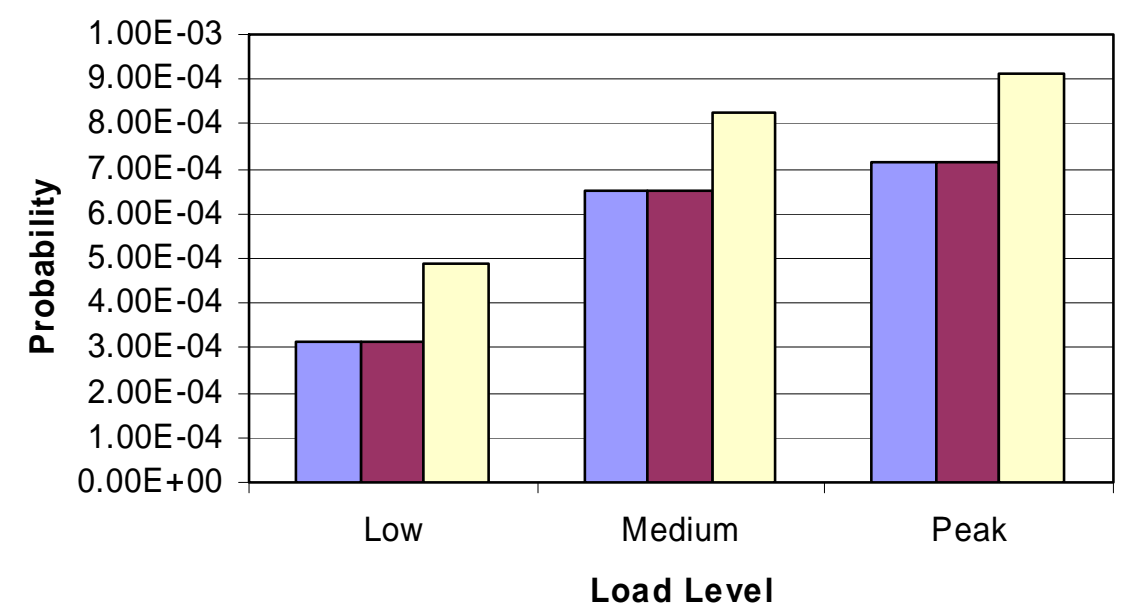

Indepent outage

$\square$ Indepent and Common-mode outages

$\square$ Independent, Common-mode, and Hidden failure outages

Figure 5.7: The probability indices of the 24-substation reliability test system under different component outage modes and load levels

(circuit outage level: 2, generating unit outage level: 0).

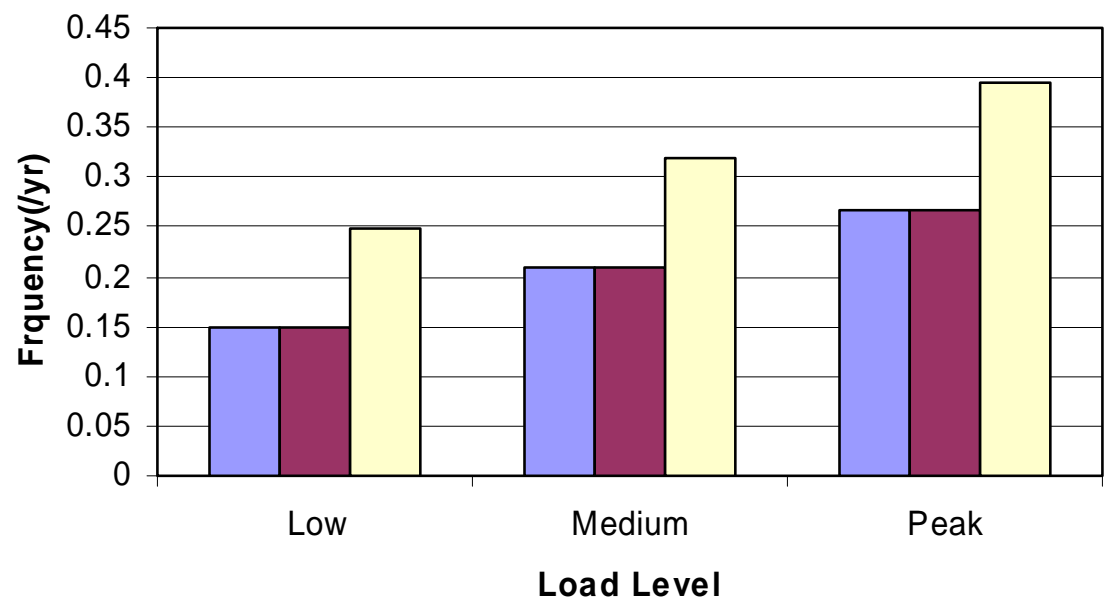

$\square$ Indepent outage

$\square$ Indepent and Common-mode outages

$\square$ Independent, Common-mode, and Hidden failure outages

Figure 5.8: The frequency indices of the 24-substation reliability test system under different component outage modes and load levels

(circuit outage level: 2, generating unit outage level: 0 ). 
Table 5.14: The reliability assessment of the 24-substation reliability test system (circuit outage level: 0).

\begin{tabular}{|c|l|c|c|}
\hline \multirow{2}{*}{$\begin{array}{c}\text { Load } \\
\text { Level }\end{array}$} & \multirow{2}{*}{$\begin{array}{c}\text { Reliability } \\
\text { Index }\end{array}$} & \multicolumn{2}{|c|}{ Generating Unit Independent Outage level } \\
\cline { 3 - 4 } Peak & Probability & 1 & 2 \\
\cline { 2 - 4 } & Frequency (/yr) & 0.0620 & 0.2163 \\
\cline { 2 - 4 } & Duration (hrs) & 8.6007 & 36.8293 \\
\hline \multirow{3}{*}{ Medium } & Probability & 63.1009 & 51.4594 \\
\cline { 2 - 4 } & Frequency (/yr) & 0.0186 & 0.0660 \\
\cline { 2 - 4 } & Duration (hrs) & 2.1617 & 9.6035 \\
\hline \multirow{3}{*}{ Low } & Probability & 75.5313 & 60.2411 \\
\cline { 2 - 4 } & Frequency (/yr) & 0.0060 & 0.0207 \\
\cline { 2 - 4 } & Duration (hrs) & 1.0579 & 4.8271 \\
\hline
\end{tabular}

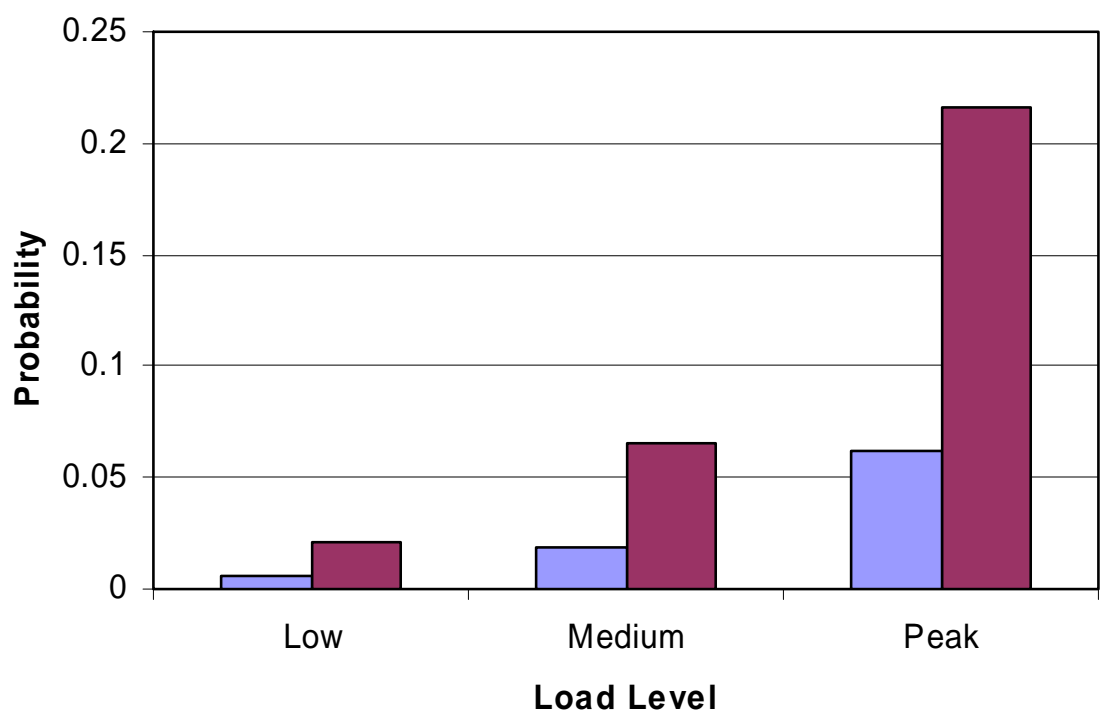

Generating unit outage level $1 \square$ Generating unit outage level 2

Figure 5.9: The probability indices of the 24-substation reliability test system under different load levels (circuit outage level: 0, generating unit outage levels: 1 and 2). 


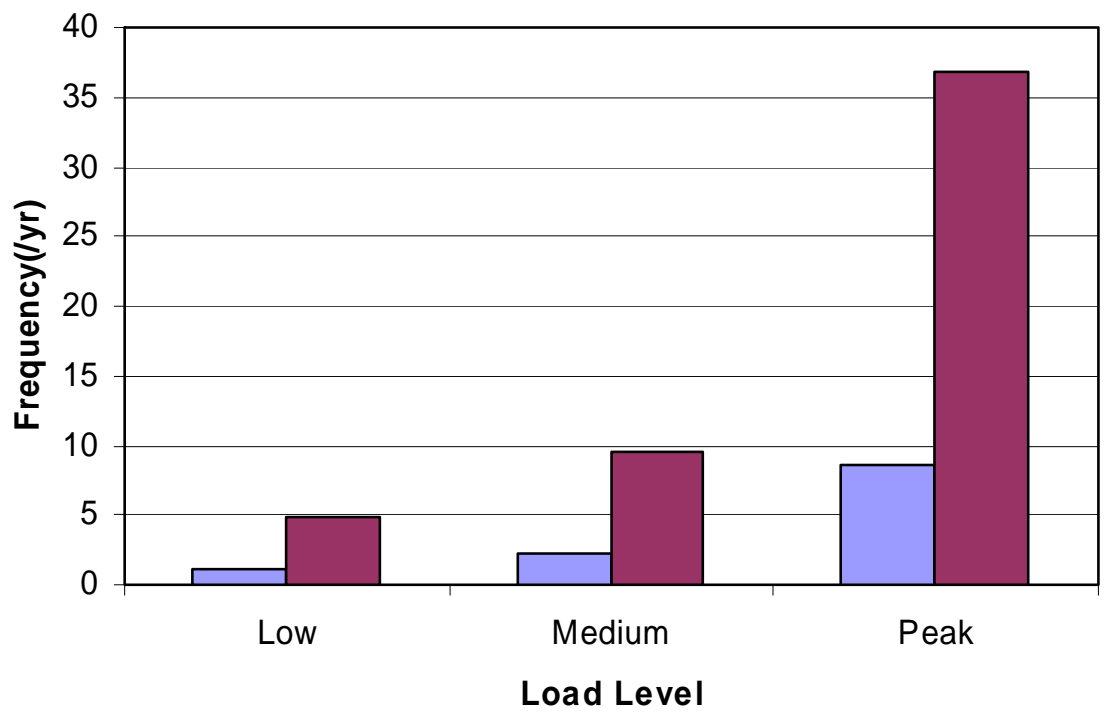

Generating unit outage level $1 \square$ Generating unit outage level 2

Figure 5.10: The frequency indices of the 24-substation reliability test system under different load levels (circuit outage level: 0, generating unit outage levels: 1 and 2).

Table 5.15: The reliability assessment of the 24-substation reliability test system (circuit outage level: 1, generating unit outage level: 1).

\begin{tabular}{|c|l|c|c|c|}
\hline \multirow{4}{*}{$\begin{array}{c}\text { Load } \\
\text { Level }\end{array}$} & \multicolumn{1}{|c|}{$\begin{array}{c}\text { Reliability } \\
\text { Index }\end{array}$} & Independent & $\begin{array}{c}\text { Independent and } \\
\text { Common-mode }\end{array}$ & $\begin{array}{c}\text { Independent, Common- } \\
\text { mode, and Hidden } \\
\text { Failure }\end{array}$ \\
\cline { 3 - 5 } & Probability & 0.0787 & 0.0788 & 0.0795 \\
\cline { 2 - 5 } Peak & Frequency (/yr) & 8.4781 & 8.4904 & 8.8134 \\
\cline { 2 - 5 } & Duration (hrs) & 81.3246 & 81.2787 & 79.0507 \\
\hline \multirow{4}{*}{ Medium } & Probability & 0.02078 & 0.02088 & 0.02141 \\
\cline { 2 - 5 } & Frequency (/yr) & 2.7722 & 2.7899 & 3.0887 \\
\cline { 2 - 5 } & Duration (hrs) & 65.6655 & 65.5649 & 60.7159 \\
\hline \multirow{3}{*}{ Low } & Probability & 0.007536 & 0.007538 & 0.0081 \\
\cline { 2 - 5 } & Frequency (/yr) & 1.7453 & 1.7458 & 2.0406 \\
\cline { 2 - 5 } & Duration (hrs) & 37.8227 & 37.8254 & 34.6066 \\
\hline
\end{tabular}




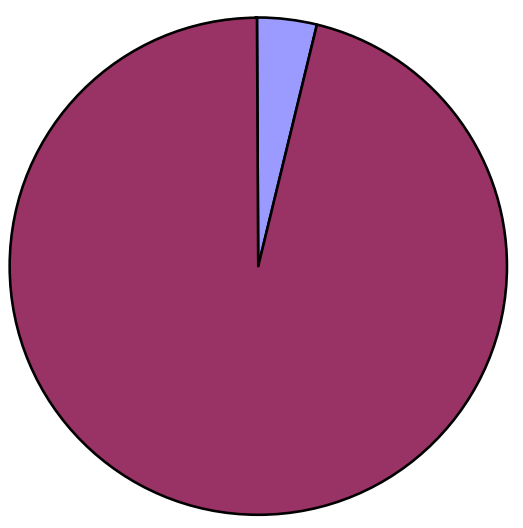

Transmission circuit outage contribution: $4.1 \%(3.04 \mathrm{e}-4)$

Generating unit outage contribution: $95.9 \%$ $(0.00723)$

Figure 5.11: The contributions to the probability index from transmission circuit outages and generating unit outages under the independent outage mode and the low load level for the 24-substation reliability test system.

Table 5.16: The reliability assessment of the 73-substation reliability test system (circuit outage level: 1 , generating unit outage level: 0 ).

\begin{tabular}{|c|c|c|c|c|}
\hline \multirow{2}{*}{$\begin{array}{l}\text { Load } \\
\text { Level }\end{array}$} & \multirow{2}{*}{$\begin{array}{l}\text { Reliability } \\
\text { Index }\end{array}$} & \multicolumn{3}{|c|}{ Outage Mode(s) } \\
\hline & & Independent & $\begin{array}{l}\text { Independent and } \\
\text { Common-mode }\end{array}$ & $\begin{array}{c}\text { Independent, Common- } \\
\text { mode, and Hidden } \\
\text { Failure }\end{array}$ \\
\hline \multirow{3}{*}{ Peak } & Probability & $5.3114 \mathrm{e}-5$ & $5.3120 \mathrm{e}-5$ & $6.7657 e-5$ \\
\hline & Frequency (/yr) & 0.0242 & 0.0242 & 0.0324 \\
\hline & Duration (hrs) & 19.2585 & 19.2569 & 18.3109 \\
\hline \multirow{3}{*}{ Medium } & Probability & $4.0715 \mathrm{e}-5$ & $4.0720 \mathrm{e}-5$ & $5.2900 \mathrm{e}-5$ \\
\hline & Frequency (/yr) & 0.0138 & 0.0138 & 0.0212 \\
\hline & Duration (hrs) & 25.8028 & 25.7982 & 21.8642 \\
\hline \multirow{3}{*}{ Low } & Probability & $3.9729 \mathrm{e}-5$ & $3.9731 \mathrm{e}-5$ & 5.0713e-5 \\
\hline & Frequency (/yr) & 0.0128 & 0.0128 & 0.0195 \\
\hline & Duration (hrs) & 27.1420 & 27.1403 & 22.8242 \\
\hline
\end{tabular}




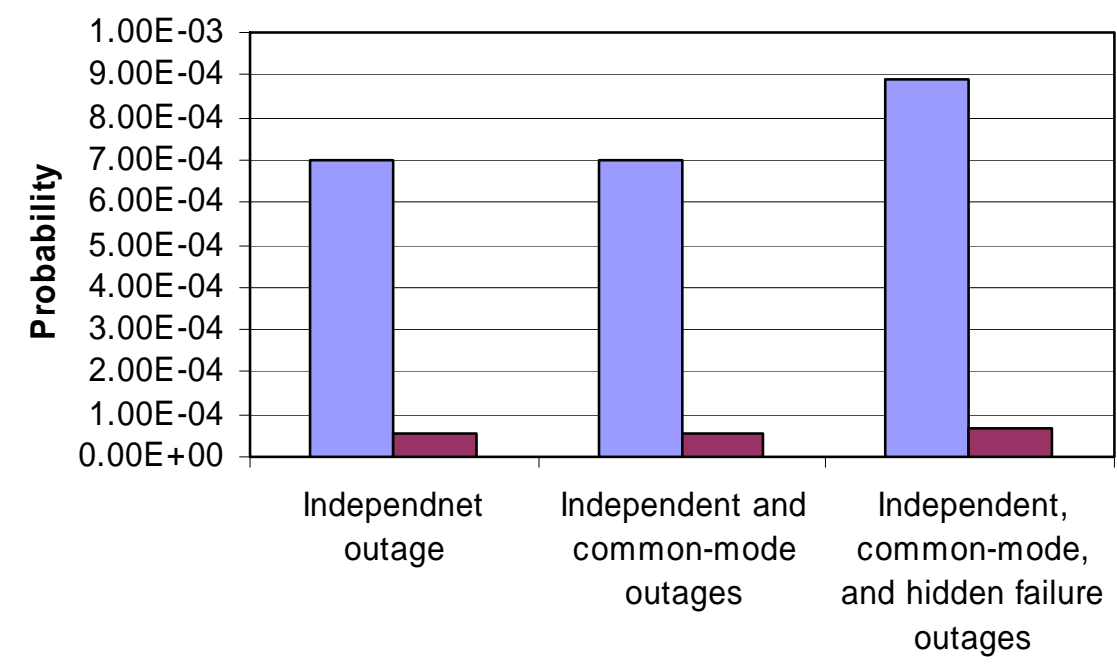

Peak Load Level

$\square$ 24-substation (single area) system $\square$ 73-substation (multi-area) system

Figure 5.12: The comparison of probability indices of the 24-substation system and the 73-substation system under the peak load level and different component outage modes (circuit outage level: 1 , generating unit outage level: 0 ).

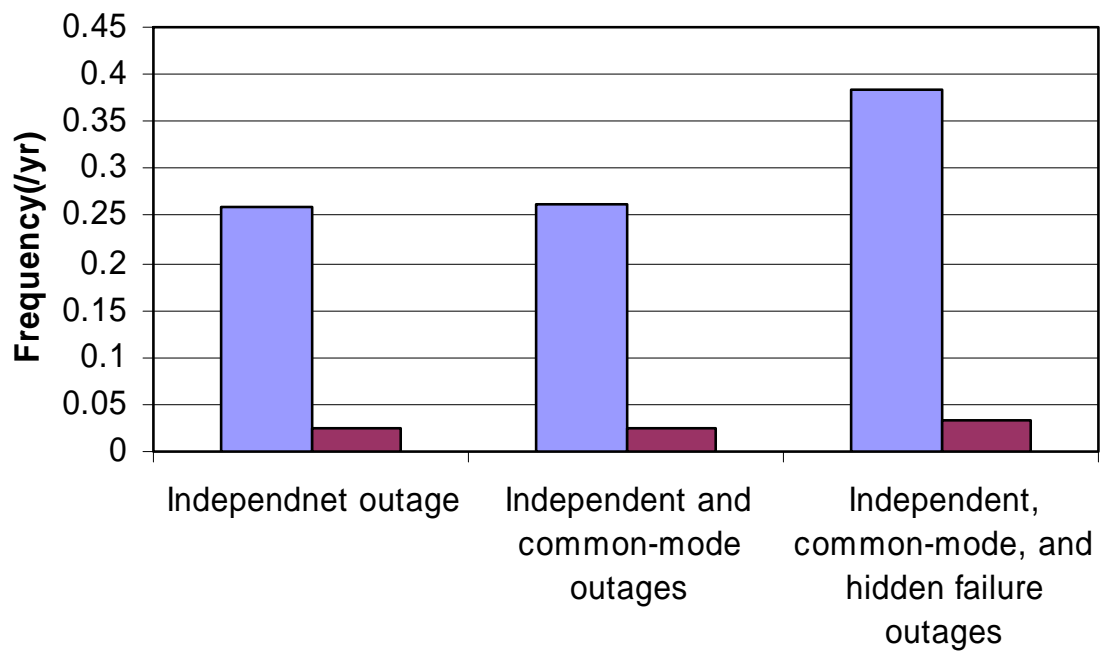

Peak Load Level

24-substation (single area) system $\square$ 73-substation (multi-area) system

Figure 5.13: The comparison of frequency indices of the 24-substation system and the 73substation system under the peak load level and different component outage modes (circuit outage level: 1, generating unit outage level: 0 ). 
Table 5.17: The reliability assessment of the 73-Substation reliability test system (circuit outage level: 0 , generating unit outage level: 1 ).

\begin{tabular}{|l|l|c|c|c|}
\hline \multicolumn{2}{|c|}{ Load Level } & Peak & Medium & Low \\
\hline \multirow{3}{*}{$\begin{array}{l}\text { Reliability } \\
\text { Index }\end{array}$} & Probability & 0.003713 & 0.002372 & $3.8988 \mathrm{e}-4$ \\
\cline { 2 - 5 } & Frequency (/yr) & 0.4196 & 0.2078 & 0.04522 \\
\cline { 2 - 5 } & Duration (hrs) & 77.5157 & 99.9914 & 75.5313 \\
\hline
\end{tabular}

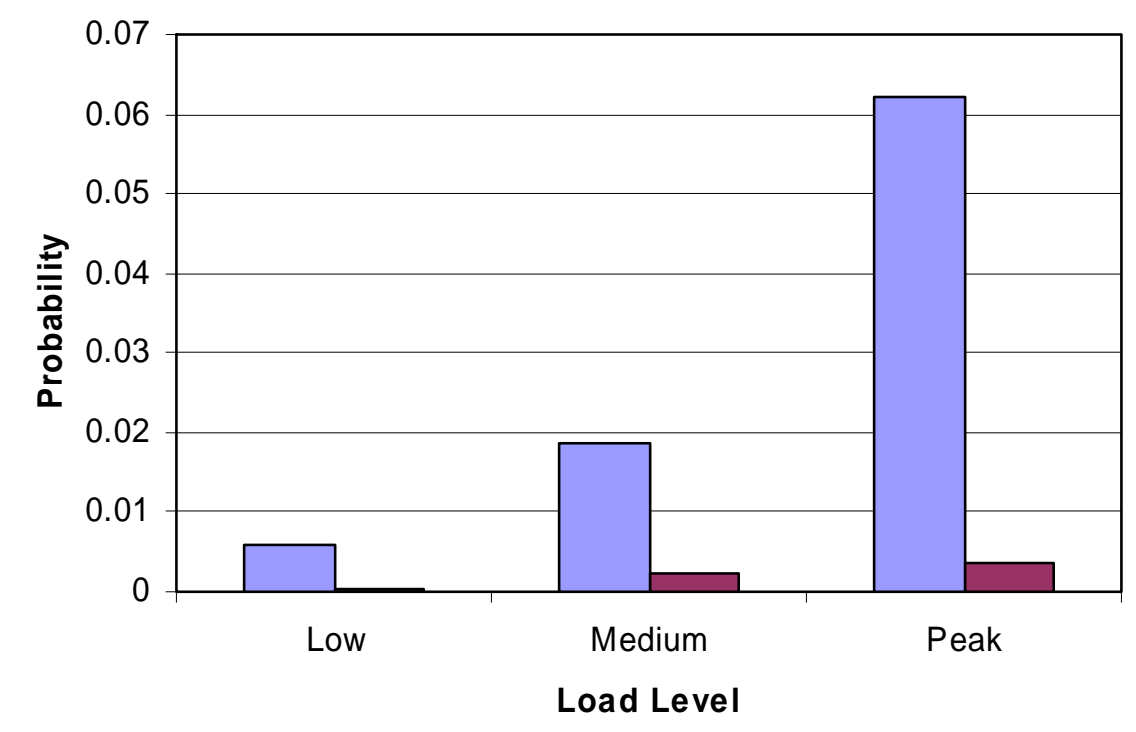

$\square$ 24-substation (single area) system $\square$ 73-substation (multi-area) system

Figure 5.14: The comparison of probability indices of the 24-substation system and the 73-substation system under the independent component outage mode and different load levels (circuit outage level: 0 , generating unit outage level: 1 ). 


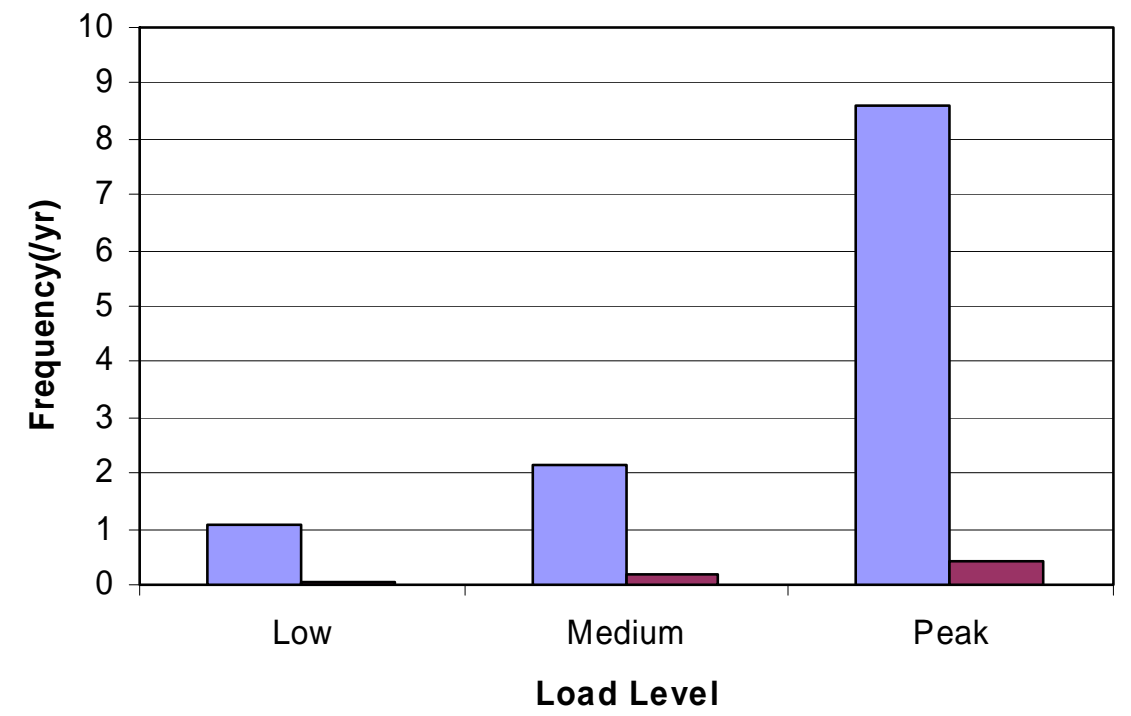

24-substation (single area) system $\square$ 73-substation (multi-area) system

Figure 5.15: The comparison of frequency indices of the 24-substation system and the 73substation system under the independent component outage mode (circuit outage level: 0, generating unit outage level: 1).

Table 5.18: Three load levels used in the reliability assessment of two systems

\begin{tabular}{|c|c|c|}
\hline Load Level & 24-substation system & 73-substation system \\
\hline Peak & 2850MW (100\%) & 9528MW (100\%) \\
\hline Medium & 2456MW (86.2\%) & 8550MW (89.7\%) \\
\hline Low & 2130MW (74.7\%) & 7572MW (79.5\%) \\
\hline
\end{tabular}




\subsection{Summary}

In this chapter, the demonstration and application of the proposed comprehensive approach for bulk power system reliability assessment are implemented based on two IEEE reliability test systems and their derivations, i.e., the circuit breaker-oriented system network models. Representative results are provided to demonstrate the effective features of advanced techniques in the proposed approach for bulk power system reliability assessment. Reliability evaluation results for two circuit breaker-oriented reliability test systems are provided and compared under different system component outage modes, outage equipment, and load levels. In the next chapter, conclusions of this research are summarized and future work is recommended. 


\section{CHAPTER VI \\ CONCLUSIONS AND RECOMMENDATIONS}

\subsection{Conclusions}

The goal of this research is to advance the state of the art in bulk power system reliability assessment. A comprehensive approach for bulk power system reliability assessment, i.e., the security-constrained adequacy evaluation (SCAE) methodology, is developed to evaluate the ability of the system in supplying the electric load while satisfying security constraints [142]. Research contributions have been made in following areas:

1. The single phase quadratized power flow (SPQPF) model is applied in the proposed SCAE methodology for bulk power system reliability assessment. Compared with the traditional power flow model, the SPQPF model yields improved contingency selection and ranking accuracy, speeds up the procedure of the effects analysis because of its faster convergence characteristics, and makes the effects analysis more realistic with its ability to model complex load characteristics.

2. An improved critical contingency selection scheme is developed to efficiently identify and rank critical contingencies with high accuracy [116, 117]. Specifically, the system state linearization approach is investigated to reduce the error introduced by the linear approximation in the traditional performance index linearization methods for contingency selection and ranking. The system state linearization approach includes 
higher-order terms in the performance index calculation procedure to trace nonlinear variations of the performance index for a post-contingency situation and therefore effectively reduce misranking in the contingency selection and ranking procedures.

3. A non-divergent optimal quadratized power flow (NDOQPF) algorithm that performs contingency effects analysis is proposed [118-120, 140, 141]. Quadratized remedial action models are developed, and the concept of the remedial action control variable is used to represent the availability and amount of system remedial actions. Compared with the traditional power flow solution procedure, the NDOQPF algorithm has the following merits:

(1) It is able to simulate contingencies in a realistic manner to capture the system response including major controls and adjustments. In addition, because of its efficiency, the overall computational effort of SCAE is reasonable;

(2) If a solution exists; it guarantees convergence; if a solution does not exist, such as when multi-level contingencies are considered and the system is severely stressed, it can provide a sub-optimal solution that may include load shedding for the system;

(3) It is applicable to both a regulated and deregulated power system environment. In particular, in a deregulated environment in which the system is more likely to be heavily stressed and may be operated in different power flow patterns from the ones it was originally designed to operate in, the NDOQPF is capable of efficiently solving the ISO/RTO operational model and providing solutions under all conditions. 
4. The impact of protection system hidden failures on bulk power system reliability assessment is investigated [134]. A circuit breaker-oriented substation model is introduced to include the detailed substation configuration as well as the protection system scheme in the system network model. In addition, the impact of advanced system real time monitoring technologies on detecting protection system hidden failures is analyzed. Also, a hidden failure effects analysis method is developed to obtain hidden failure outages following any possible initial equipment outages. The security-constrained adequacy evaluation methodology is extended to evaluate contingencies resulting from protection system hidden failures as well as other contingencies resulting from independent and common-mode outages so that the impact of protection system hidden failures on bulk power system reliability assessment is included.

5. The proposed comprehensive bulk power system reliability assessment approach is implemented in a Visual $\mathrm{C}++$ environment using object-oriented techniques and is applied to evaluate the reliability of two reliability test systems.

\subsection{Recommendations for Future work}

Based on the conclusions above, recommendations for future research directions are listed as follows: 
1. More potentially efficient algorithms for solving large-scale optimization problems, such as the interior point method, should be implemented and evaluated to improve the performance of the optimization problem solution.

2. The proposed approach will be applied to solve the ISO/RTO operational model to demonstrate its effectiveness in deregulated power systems.

3. The proposed approach will be extended to integrate the security assessment, which involves evaluating system behavior while including transient stability as well as cascading sequences after system disturbances.

4. The performance of the software that implements the proposed approach in the Visual C++ environment using object-oriented techniques will be further improved. 


\section{REFERENCES}

[1] M. P. Bhavaraju, R. Billinton, R. E. Brown, J. Endrenyi, W. Li, A. P. Meliopoulos, and C. Singh, IEEE Tutorial on Electric Delivery System Reliability Evaluation, IEEE Power Engineering Society General Meeting, 2005.

[2] R. Billinton and R. N. Allan, Reliability Evaluation of Power Systems, NY: Plenum Press, 1996.

[3] R. Billinton and W. Li, Reliability Assessment of Electric Power Systems Using Monte Carlo Methods, NY: Plenum Press, 1994.

[4] J. Endrenyi, Reliability Modeling in Electric Power System, NY: John Wiley \& Sons Ltd., 1978.

[5] A. P. Meliopoulos, R. Kovacs, N. J. Balu, M. Lauby, N. D. Reppen, M. P. Bhavaraju, and R. Billinton, "A Probabilistic Method for Transmission Planning," The $2^{\text {nd }}$ International Symposium on Probability Method Applied in Power Systems, Oakland, USA, September 1988.

[6] Task Force on Bulk Power System Reliability, "Bulk Power System Reliability Concepts and Applications,” IEEE Transactions on Power Systems, Vol. 3, No. 1, pp. 109-117, February 1988.

[7] B. Porretta and E. G. Neudorf, "Conceptual Framework for Evaluation and Interpretation of the Reliability of the Composite Power System," IEEE Transactions on Power Systems, Vol. 10, No. 2, pp. 1094-1103, May 1995.

[8] R. Billinton, L. Salvaderi, J. D. McCalley, H. Chao, T. Seitz, R. N. Allen, J. Odom, and C. Fallon, "Reliability Issues in Today's Electric Power Utility Environment,” IEEE Transactions on Power Systems, Vol. 12, No. 4, pp. 17081714, November 1997.

[9] K. N. Zadeh, R. C. Meyer, and G. Cauley, "Practices and New Concepts in Power System Control," IEEE Transactions on Power Systems, Vol. 11, No. 1, pp. 3-10, February 1996.

[10] Z. Alaywan and J. Allen, "California Electric Restructuring,” IEEE Transactions on Power Systems, Vol. 13, No. 4, pp. 1445-1452, November 1998. 
[11] J. Katzel, "Managing Energy in a Deregulated Environment: Opportunity or Chaos?” Plant Engineering, pp. 36-42, January 1999.

[12] H. B. Puttgen, D.R. Volzka, and M. I. Olken, "Restructuring and Re-regulation of the US Electric Utility Industry,” IEEE Power Engineering Review, Vol. 21, No. 2, pp. 8-10, February 2001.

[13] Y. V. Makarov and R. C. Hardiman, "Risk, Reliability, Cascading and Restructuring," IEEE Power Engineering Society General Meeting, Vol. 3, pp. 1417-1429, July 2003.

[14] W. J. Lee and C. H. Lin, "Utility Deregulation and Its Impact on the Industrial Power Systems," Industrial and Commercial Power Systems Technical Conference, pp. 217-222, May 1997.

[15] L. H. Fink and P. J. M. V. Son, "On System Control within a Restructured Industry,” IEEE Transactions on Power Systems, Vol. 18, No. 2, pp. 611-616, May 1998.

[16] J. W. M. Cheng, D. T. McGillis, and F. D. Galiana, "Power System Reliability in a Deregulated Environment," Conference on Electrical and Computer Engineering, Vol. 2, pp. 765-768, Mar. 2000.

[17] J. De La Ree, Y. Liu, L. Mili, A. G. Phadke, and L. Dasilva, "Catastrophic Failures in Power Systems, Cause, Analysis, and Countermeasures," IEEE Proceedings, Vol. 93, No. 5, pp. 956-964, 2005.

[18] R. Allan and R. Billinton, "Power System Reliability and its Assessment. Part 1 Background and Generating Capacity,” Power Engineering Journal, Vol. 6, No. 4, pp. 191-196, July 1992.

[19] R. Allan and R. Billinton, "Power System Reliability and its Assessment. Part 2 Composite generation and transmission systems," Power Engineering Journal, Vol. 6, No. 6, pp. 291-297, November 1992.

[20] B. L. Silverstein and D. M. Porter, "Contingency Ranking for Bulk System Reliability Criteria,” IEEE Transactions on Power Systems, Vol. 7, No. 3, pp. 956-964, August 1992.

[21] B. E. Arporn and A. Karunanoon, "Reliability Evaluation in Electrical Power Generation with Uncertainty Modeling by Fuzzy Number," IEEE Power Engineering Society Summer Meeting, Vol.4, pp. 2051-2056, July 2000. 
[22] J. T. Saraiva, "Reliability Evaluation of Generation/Transmission Power Systems Including Fuzzy Data," 1996 IEEE International Symposium on Circuits and Systems, Vol. 1, pp. 609-612, May 1996.

[23] J. T. Saraiva, V. Miranda, and L. M. V. G. Pinto, "Generation/Transmission Power System Reliability Evaluation by Monte-Carlo Simulation Assuming a Fuzzy Load Description,” IEEE Transactions on Power Systems, Vol. 11, No. 2, pp. 690-695, May 1996.

[24] J. T. Saraiva and A. V. Sousa, "New Advances in Integrating Fuzzy Data in Monte Carlo Simulation to Evaluate Reliability Indices of Composite Power Systems,” Electrotechnical Conference, Mditerranean, Vol. 2, pp. 1084-1088, May 1998.

[25] V. Miranda and J. T. Saraiva, "Fuzzy Modeling of Power System Optimal Load Flow," IEEE Transactions on Power Systems, Vol. 7, No. 2, pp. 843-849, May 1992.

[26] J. T. Saraiva, V. Miranda, and L. M. V. G. Pinto, "Impact on Some Planning Decisions From a Fuzzy Modeling of Power Systems," IEEE Transactions on Power Systems, Vol. 9, No. 2, pp. 819-825, May 1994.

[27] M. T. Schilling, R. Billinton, A. M. L. D. Silva, and M. A. E. Kady, "Bibliography on Composite System Reliability 1964-1988," IEEE Transactions on Power Systems, Vol. 4, No. 3, pp. 1122-1132, August 1989.

[28] R. N. Allan, R. Billinton, S. M. Shahidehpour, and C. Singh, "Bibliography on the Application of Probability Methods in Power System Reliability Evaluation 19821987,” IEEE Transactions on Power Systems, Vol. 3, No. 4, pp. 1555-1564, November 1988.

[29] R. N. Allan, R. Billinton, A. M. Breipohl, and C. H. Grigg, "Bibliography on the Application of Probability Methods in Power System Reliability Evaluation 19871991,” IEEE Transactions on Power Systems, Vol. 9, No. 1, pp. 41-49, February 1994.

[30] R. N. Allan, R. Billinton, A. M. Breipohl, and C. H. Grigg, "Bibliography on the Application of Probability Methods in Power System Reliability Evaluation 19921996,” IEEE Transactions on Power Systems, Vol. 14, No. 1, pp. 51-57, February 1999.

[31] R. Billinton, M. F. Firuzabad, and L. Bertling, "Bibliography on the Application of Probability Methods in Power System Reliability Evaluation 1996-1999,” IEEE Transactions on Power Systems, Vol. 14, No. 1, pp. 595-602, February 1999. 
[32] E. Liu, "Technology Integration-Computing and Analytical Methods," Power Engineering Society Summer meeting, Vol. 2, July 1999.

[33] EPRI Report EL-2526, “Transmission System Reliability Methods,” July 1982.

[34] EPRI Report EL-5291, "Reliability Evaluation for Large-Scale Bulk Transmission Systems," Comparative Evaluation, Method Development, and Recommendation, Vol. 1, January 1988.

[35] R. Billinton and A. Sankarakrishnan, "A Comparison of Monte Carlo Simulation Techniques for Composite Power System Reliability Assessment,” Proceedings of IEEE Conference on Communications, Power, and Computing, Vol.1, pp. 145150, May 1995.

[36] M. E. Khan and R. Billinton, "A Hybrid Model for Quantifying Different Operating States of Composite Power Systems,” IEEE Transactions on Power Systems, Vol. 7, No. 1, pp. 187-193, February 1992.

[37] Y. Guo, Y. Xi, K. Xiao, and H. Yang, "Composite System Reliability Evaluation Based on Monte-Carlo Simulation Combined with Outages Screening,” IEEE Transactions on Power Systems, Vol. 14, No. 1, pp. 785-790, May 1999.

[38] X. Luo, C. Singh, and A. D. Patton, "Power System Reliability Evaluation Using Self Organizing Map,” IEEE Power Engineering Society Winter Meeting, Vol. 2, pp. 1103-1108, January 2000.

[39] R. Billinton and E. Khan, "A Security Based Approach to Composite Power System Reliability Evaluation," IEEE Transactions on Power Systems, Vol. 7, No. 1, pp. 65-72, February 1992.

[40] R. Billinton and W. Zhang, "State Extension in Adequacy Evaluation of Composite Power Systems --- Concept and Algorithm,” Electric Power Systems Research, Vol. 14, pp.189-195, 1998.

[41] R. Billinton and W. Zhang, "State Extension for Adequacy Evaluation of Composite Power Systems --- Applications," IEEE Transactions on Power Systems, Vol. 15, No. 1, pp. 427-432, February 2000.

[42] R. Billinton and S. K. Agarwal, "Examination of severe contingencies in a small area of a large composite power system using adequacy equivalent," IEE Proceedings, Vol. 137, No. 2, pp.107-114, March 1990. 
[43] S. Kumar and R. Billinton, "Adequacy Equivalents in Composite Power System Evaluation,” IEEE Transactions on Power Systems, Vol. 3, No. 3, pp. 1167-1173, August 1998.

[44] R. Billinton and W. Zhang, "Enhanced Adequacy Equivalent for Composite Power System Reliability Evaluation,” IEE Proc.-Gener. Transm. Distrib., Vol. 143, No.5, September 1996.

[45] W. Zhang and R. Billinton, "Application of an Adequacy Equivalent Method in Bulk Power System Reliability Evaluation," IEEE Transactions on Power Systems, Vol. 13, No. 2, pp. 661-666, May 1998.

[46] H. A. M. Maghraby and R. N. Allan, "Application of DC Equivalents to the Reliability Evaluation of Composite Power Systems," IEEE Transactions on Power Systems, Vol. 14, No. 1, pp. 355-361, February 1999.

[47] J. Zaborszky and F. W. Whang, "Fast Contingency Evaluation Using Concentric Realization," IEEE Transactions on Power Apparatus and Systems, Vol. PAS-99, No. 1, pp. 28-36, January/February 1980.

[48] V. Brandwajn, "Efficient Bounding Method for Linear Contingency Analysis," IEEE Transactions on Power Systems, Vol. PWRS-3, No. 1, pp. 38-43, February 1988.

[49] V. Brandwajn and M. G. Lauby, "Complete Bounding for AC Contingency Analysis," IEEE Transactions on Power Systems, Vol. PWRS-4, No. 2, pp. 724729, May 1990.

[50] A. P. S. Meliopoulos and C. Cheng, "A Hybrid Contingency Selection Method," Proceedings of the $10^{\text {th }}$ Power System Computation Conference, Austria, pp. 605612, August 1994.

[51] S. Kang and A. P. S. Meliopoulos, "Analytical Approach for the Evaluation of Actual Transfer Capability in a Deregulated Environment," Proceedings of the $32^{\text {nd }}$ Annual North American Power Symposium, 1999.

[52] A. P. Sakis Meliopoulos, Power System Modeling, Analysis and Control, Georgia Institute of Technology, 2002.

[53] G. C. Ejebe and B. F. Wollenberg, "Automatic Contingency Selection,” IEEE Transactions on Power Apparatus and Systems, Vol. PAS-98, No.1, pp.92-104, January/February 1979. 
[54] A. P. Sakis Meliopoulos, C. S. Cheng, and F. Xia, "Performance Evaluation of Static Security Analysis Methods,” IEEE Transactions on Power Systems, Vol. 9, No. 3, pp. 1441-1449, August 1994.

[55] G. Irisarri, A. M. Sasson, and D. Levner, "Automatic Contingency Selection for On-Line Security Analysis-Real Time Tests," IEEE Transactions on Power Apparatus and Systems, Vol. PAS-98, No.5, pp.1552-1559, September/October 1979.

[56] A. J. Wood and B. F. Wollenberg, Power Generation, Operation, and Control, John Wiley \& Sons, Inc. 1984.

[57] IEEE Committee Report, "Parallel Processing in Power Systems Computation," IEEE Transactions on Power Systems, Vol. 7, No. 2, pp. 629-638, May 1992.

[58] C. L. T. Borges, D. M. Falcao, J. C. O. Mello, and A. C. G. Melo, "Composite Reliability Evaluation by Sequential Monte Carlo Simulation on Parallel and Distributed Processing Environment," IEEE Transactions on Power Systems, Vol. 16, No. 2, pp. 203-209, May 2001.

[59] C. Lemaitre and B. Thomas, "Two Applications of Parallel Processing in Power System Computation,” IEEE Transactions on Power Systems, Vol. 11, No. 1, pp. 246-253, February 1996.

[60] M. J. Teixeira, H. J. C. P. Pinto, M. V. F. Pereira, and M. F. McCoy, "Developing Concurrent Processing Applications to Power System Planning and Operations," IEEE Transactions on Power Systems, Vol. 5, No. 2, pp. 659-664, May 1990.

[61] N. Gubbala and C. Singh, "Models and Considerations for Parallel Implementation of Monte Carlo Simulation Methods for Power System Reliability Evaluation,” IEEE Transactions on Power Systems, Vol. 10, No. 2, pp. 779-787, May 1995.

[62] D. J. Boratynska-Stadnicka, M. G. Lauby, and J. E. V. Ness, "Converting an Existing Computer Code to a Hypercube Computer," Power Industry Computer Application Conference, pp. 394-399, May 1989.

[63] C. Singh and J. Mitra, "Composite System Reliability Evaluation using State Space Pruning,” IEEE Transactions on Power Systems, Vol. 12, No. 1, pp. 471479, February 1997. 
[64] D. P. Clancy, G. Gross, and F. F. Wu, "Probabilistic Flows for Reliability Evaluation of Multi-area Power System Interconnections," Electric Power \& Energy Systems, Vol. 5, No. 2, pp.101-114, April 1983.

[65] C. Singh, and Z. Deng, "A New Algorithm for Multi-Area Reliability EvaluationSimultaneous Decomposition Simulation Approach,” Electric Power \& Energy Systems, Vol. 21, No. 2, pp.129-136, April. 1983.

[66] Z. Deng and C. Singh, "A New Approach to Reliability Evaluation of Interconnected Power Systems Including Planned Outages and Frequency Calculations," IEEE Transactions on Power Systems, Vol. 7, No. 2, pp. 734-743, May 1992.

[67] J. Mitra and C. Singh, "Incorporating the DC Load Flow Model in the Decomposition-Simulation Method of Multi-Area Reliability Evaluation,” IEEE Summer Power Meeting, July 1995.

[68] J. Mitra, Models for Reliability Evaluation of Multi-Area and Composite, Ph.D. Dissertation, Texas A\&M University, TX, 1997.

[69] J. Mitra and C. Singh, "Pruning and Simulation for Determination of Frequency and Duration Indices of Composite Power Systems," IEEE Transactions on Power Systems, Vol. 14, No. 3, pp. 899-905, August 1999.

[70] K. Bae and J. S. Thorp, "An Importance Sampling Application: 179 Bus WSCC System under Voltage Based Hidden Failures and Relay Mis-operations," International Conference on System Science, January 1997.

[71] A. G. Phadke and J. S. Thorp, "Expose Hidden Failures to Prevent Cascading Outages," IEEE Computer Application in Power, pp. 20-23, July 1996.

[72] M. V. F. Pereira, M. E. P. Maceira, and L. M. V. G. Pinto, "Combining Analytical Models and Monte-Carlo Techniques in Probabilistic Power System Analysis," IEEE Transactions on Power Systems, Vol. 7, No. 1, pp. 265-272, February 1992.

[73] M. E. Khan and R. Billinton, "A Hybrid for Quantifying Different Operating States of Composite Power Systems," IEEE Transactions on Power Systems, Vol. 7, No. 1, pp. 187-193, February 1992.

[74] A. C. G. Melo, G. C. Oliverira, M. Morozaowski, and M. V. F. Pereira, "A Hybrid Algorithm for Monte Carlo/Enumeration Based Composite Reliability Evaluation,” Third International Conference on Probabilistic Methods Applied to Electric Power Systems, pp.70-74, July 1991. 
[75] R. Billinton and A. Jonnavithula, "Variance Reduction Techniques for Use with Sequential Monte Carlo Simulation in Bulk Power System Reliability Evaluation," Conference on Electrical and Computer Engineering, Vol. 1, pp.416419, May 1996.

[76] R. Billinton and A. Jonnavithula, "Composite System Adequacy Assessment Using Sequential Monte Carlo Simulation with Variance Reduction Techniques," IEE Proc.-Gener. Transm. Distrib., Vol. 144, No. 1, pp. 1-6, January 1997.

[77] A. Sankarakrishnan and R. Billinton, "Sequential Monte Carlo Simulation for Composite Power System Reliability Analysis with Time Varying Loads," IEEE Transactions on Power Systems, Vol. 10, No. 3, pp. 1540-1545, August 1995.

[78] C. Dornellas, M. Schilling, A. Melo, J. C. S. Souza, and M. B. Do Coutto Filho, "Combining Local and Optimised Power Flow Remedial Measures in Bulk Reliability Assessment," IEE Proc.-Gener. Transm. Distrib., Vol. 150, No. 5, September 2003.

[79] R. Billinton and E. Khan, "A Security Based Approach to Composite Power System Reliability Evaluation," IEEE Transactions on Power Systems, Vol. 7, No. 1, pp. 65 - 72, February 1992.

[80] M. E. Khan and R. Billinton, "A Hybrid Model for Quantifying Different Operating States of Composite Systems," IEEE Transactions on Power Systems, Vol. 7, No. 1, pp. 187 - 193, February 1992.

[81] R. Billinton and S. Aboreshaid, "Security Evaluation of Composite Power Systems," IEE Proc.-Gener. Transm. Distrib., Vol. 142, No. 5, pp. 511-516, September 1995.

[82] M. E. Khan, "Bulk Load Points Reliability Evaluation Using a Security Based Model," IEEE Transactions on Power Systems, Vol. 13, No. 2, pp. 456 - 463, May 1998.

[83] R. Billinton and M. E. Khan, "Security Considerations in Composite Power System Reliability Evaluation,” Third International Conference on Probabilistic Methods Applied to Electric Power Systems, pp.58-63, July 1991.

[84] D. Romero-Romero, J. A. Gomez-Hernandez and J. Robles-Garcia, "Reliability Optimization of Bulk Power Systems Including Voltage Stability," IEE Proc.Gener. Transm. Distrib., Vol. 150, No. 5, pp.561-566, September 2003. 
[85] D. T. Y. Cheng, "The Challenges of Using an Optimal Power Flow,” IEEE Power Engineering Review, pp. 62-63, October 1998.

[86] Y. Chao, Nondivergent and Optimal Power Flow: A Unified Approach, Ph.D. Thesis, Georgia Institute of Technology, 1991.

[87] M. Huneadult and F. D. Galiana, "A Survey of the Optimal Power Flow Literature," IEEE Transactions on Power Systems, Vol. 6, No.2, pp. 762-770, May 1991.

[88] J. L. Carpentier and G. Cotto, "Optimal Power Flows: Uses, Methods and Developments,” Proc. of IFAC Conference, 1985.

[89] T. S. Dillon, "Computational Methods for Solution of the Optimal Power Flow Problem," Fourth International Symposium on Large Engineering Systems, Calgary, 1982.

[90] H. H. Happ, "Optimal Power Dispatch - A Comprehensive Survey," IEEE Transactions on PAS, Vol. 96, pp. 841-854, 1977.

[91] A. M. Sasson and H. M. Merril, "Some Application of Optimization Techniques to Power System Problems,” Proc. of IEEE, Vol. 62, No. 7, pp. 959-972, 1974.

[92] B. Stott, O. Alsac, and J. L. Marinho, "The Optimal Power Flow Problem," Electric Power Problems: The Mathematic Challenge, pp. 327-351, SIAM 1980.

[93] B. Scott, O. Alsac, and A. J. Monticelli, "Security Analysis and Optimization," Proc. Of IEEE, Vol. 75, No. 12, pp. 1623-1644, 1987.

[94] S. N. Talukdar and F. F. Wu, "Computer Aided Dispatch for Electric Power Systems,” Proc. of IEEE, Vol. 69, No. 10, October 1981.

[95] J. A. Momoh, M. E. El-Hawary, and R. Adapa, "A Review of Selected Optimal Power Flow Literature to 1993 Part 1: Nonlinear and Quadratic Programming Approaches,” IEEE Transactions on Power Systems, Vol. 14, No.1, pp. 96-104, February 1999.

[96] O. Alsac and B. Scott, "Optimal Load Flow with Steady-State Security," IEEE Transactions on Power Apparatus and Systems, Vol. PAS-93, pp. 745-751, May/June 1974.

[97] K. R. C. Mamandur and R. D. Chenoweth, "Optimal Control of Reactive Power Flow for Improvements in Voltage Profiles and for Real Power Loss 
Minimization,” IEEE Transactions on Power Apparatus and Systems, Vol. PAS100, No. 7, pp. 3185-3194, July 1981.

[98] B. Scott, O. Alsac, and A. J. Monticelli, "Security Analysis and Optimization," Proceeding of the IEEE, Vol. 75, No.12, pp. 1623-1644, December 1987.

[99] E. Hobson, "Network Constrained Reactive Power Control Using Linear Programming," IEEE Transactions on Power Apparatus and Systems, Vol. PAS99, No. 3, pp. 867-877, May/June 1980.

[100] J. C. Kaltenbach and L. P. Hajdu, "Optimal Corrective Rescheduling for Power System Security,” IEEE Transactions on Power Apparatus and Systems, Vol. PAS-90, No. 2, pp. 843-851, March/April 1971.

[101] R. C. Degeneff, W. Neugebauer, C. H. Saylor, and S. L. Corey, "SecurityConstrained Optimization: An Added Dimension in Utility Systems Optimal Power Flow,” IEEE Computer Applications in Power, Vol. 1, No.4, pp. 26-30, October 1988.

[102] G. Schnyder and H. Glavitsch, "Security Enhancement using an Optimal Switching Power Flow,” Proc. of PICA Conference, pp.25-32, 1987.

[103] D. Gan, R. J. Thomas, and R. D. Zimmerman, "Stability-Constrained Optimal Power Flow,” IEEE Transactions on Power Systems, Vol. 15, No. 2, pp. 535-540, May 2000.

[104] Y. Yuan, J. K, and H. Sasaki, "A Solution of Optimal Power Flow With Multicontingency Transient Stability Constraints," IEEE Transactions on Power Systems. Vol. 18, No. 3, pp. 1094-1099, Aug. 2003.

[105] A. M. L. Silva, J. Endrenyi, and L. Wang, "Integrated Treatment of Adequacy and Security in Bulk Power System Reliability Evaluation," IEEE Transactions on Power Systems, Vol. 3, No. 1, pp. 275-285, March 1993.

[106] A. M. Rei, A. M. L. Silva, J. L. Jardim, and J. C. O. Mello, "Static and Dynamic Aspects in Bulk Power System Reliability Evaluation," IEEE Transactions on Power Systems, Vol. 15, No. 1, pp. 189-195, February 2000.

[107] V. A. Levi, J. M. Nahman, and D. P. Nedic, "Security Modeling for Power System Reliability Evaluation,” IEEE Transactions on Power Systems, Vol. 16, No. 1, pp. 29-37, February 2001. 
[108] B. Porretta, D. L. Kiauel, G. A. Hamoud, and E. G. Neudorf, "A Comprehensive Approach for Adequacy and Security Evaluation of Bulk Power Systems,” IEEE Transactions on Power Systems, Vol. 6, No. 2, pp. 433-441, May 1991.

[109] R. Billinton and Saleh Aboreshaid, "Voltage Stability Considerations in Composite Power System Reliability Evaluation,” IEEE Transactions on Power Systems, Vol. 13, No. 2, pp. 655-660, May 1998.

[110] L. Chen, Y. Guo, and K. Xiao, "Reliability Evaluation of Composite Systems Considering Voltage Stability Problems," International Conference on Power System Technology, Vol. 2, pp. 1489-1493, August 1998.

[111] J. A. Momoh, Y. V. Makarov, and W. Mittelstadt, "A Framework of Voltage Stability Assessment in Power System Reliability Analysis,” IEEE Transactions on Power Systems, Vol. 14, No. 2, pp. 484-491, May 1999.

[112] N. Amjady, "A Framework of Reliability Assessment with Consideration Effect of Transient and Voltage Stability," IEEE Transactions on Power Systems, Vol. 19, No. 2, pp. 1005-1014, May 2004.

[113] S. W. Kang, A New Approach for Power Transaction Evaluation and Transfer Capability Analysis, Ph. D. Thesis, Georgia Institute of Technology, 2001.

[114] PSERC Project Report, "Comprehensive Power System Reliability Assessment," December 2003.

[115] S. W. Kang and A. P. Sakis Meliopoulos, "Contingency Selection via Quadratized Power Flow Sensitivity Analysis," IEEE Power Engineering Society Summer Meeting, Vol.3, pp.1494-1499, July 2002.

[116] G. K. Stefopoulos, F. Yang, and A. P. Sakis Meliopoulos, "An Improved Contingency Ranking Method”, $35^{\text {th }}$ North American Power Symposium, October 2003.

[117] G. K. Stefopoulos, F. Yang, G. J. Cokkinides, and A. P. Sakis Meliopoulos, "Advanced Contingency Selection Methodology," $37^{\text {th }}$ North American Power Symposium, October 2005.

[118] F. Yang, A. P. Meliopoulos, G. J. Cokkinides, and G. K. Stefopoulos, "A Bulk Power System Reliability Assessment Methodology," $8^{\text {th }}$ International Conference on Probabilistic Methods Applied to Power Systems, September 2004. 
[119] F. Yang, A. P. Meliopoulos, G. J. Cokkinides, and G. K. Stefopoulos, "SecurityConstrained Adequacy Evaluation of Bulk Power System Reliability,” $\underline{9^{\text {th }}}$ International Conference on Probabilistic Methods Applied to Power Systems, June 2006.

[120] F. Yang, A. P. Meliopoulos, and G. K. Stefopoulos, "Effects of Remedial Actions on LMP,” $37^{\text {th }}$ North American Power Symposium, October 2005.

[121] M. Sasson, "Power System Security and Reliability Aspects Under SMD: The New York Experience," IEEE Power Engineering Society General Meeting, pp.376 - 382, June 2004.

[122] Z. Alaywan and J. Allen, "California Electric Restructuring; A Broad Description of the Development of the California ISO,” IEEE Transactions on Power Systems, Vol. 13, No. 4, pp. 1445-1452, November 1998.

[123] S. R. Jones, "ERCOT ISO Experience with Transmission Operating and Planning Criteria in a Competitive Environment," IEEE Power Engineering Society Summer Meeting, Vol. 1, pp. 140-142, July 1999.

[124] A. L. Ott, "Experience with PJM Market Operation, System Design, and Implementation,” IEEE Transactions on Power Systems, Vol. 18, No. 2, pp. 528534, May 2003.

[125] L. H. Fink and P. J. M. V. Son, "On System Control within a Restructured Industry,” IEEE Transactions on Power Systems, Vol. 18, No. 2, pp. 611-616, May 1998.

[126] J. W. M. Cheng, D. T. McGillis, F. D. Galiana, "Power System Reliability in a Deregulated Environment," Conference on Electrical and Computer Engineering, Vol. 2, pp. 765-768, March 2000.

[127] S. H. Horowitz and A. G. Phadke, Power System Relaying, John Wiley \& Sons Inc. 1992.

[128] D. C. Elizondo and J. D. L. Ree, "Analysis of Hidden Failures of Protection Schemes in Large Interconnected Power Systems,” IEEE Power Engineering Society General Meeting, 2004.

[129] A. G. Phadke and J. S. Thorp, "Expose Hidden Failures to Prevent Cascading Outages,” IEEE Computer Application in Power, Vol. 9, No. 3, pp. 20-23, 1996. 
[130] D. C. Elizondo, J. D. L. Ree, A. G. Phadke, and S. Horowitz, "Hidden Failures in Protection Systems and Their Impact on Wide-Area Disturbance,” IEEE Power Engineering Society Winter Meeting, Vol. 2, pp. 710-714, 2001.

[131] S. Tamronglak, Analysis of Power System Disturbance due to Relay Hidden Failures, Ph. D. Dissertation, Virginal Polytechnic Institute State University, 1994.

[132] D. C. Elizondon, Hidden Failures in Protection Systems and its Impact on WideArea Disturbances, M.S.E.E. Thesis, Virginal Polytechnic Institute State University, 2000.

[133] PSERC Project Report, "New Implications of Power System Fault Current Limits,” 2005.

[134] F. Yang, A. P. Meliopoulos, G. J. Cokkinides, and Q. B. Dam, "Effects of Protection System Hidden Failures on Bulk Power System Reliability," $38^{\text {th }}$ North American Power Symposium, June 2006.

[135] J. Beaupre, M. Lehous, and P. A. Berger, "Advanced Monitoring Technologies for Substations," IEEE Conference on Transmission and Distribution Construction, Operation and Live-in Maintenance, pp. 287-292, 2000.

[136] D. Xu, R. He, P. Wang, and T. Xu, "Comparison of Several PMU Placement Algorithms for State Estimation,” IEE Conference on Developments in Power System Protection, Vol. 1, pp. 32-35, 2004.

[137] J. Bertsch, M. Zima, A. Suranyi, C. Carnal, C. Rehtanz, and M. Larsson, "Experiences with and Perspectives of the System for Wide Area Monitoring of Power System," Quality and Security of Electric Power Delivery Systems Symposium, pp. 5-9, 2003.

[138] IEEE Committee Report, “IEEE Reliability Test System,” IEEE Transactions on Power Apparatus and Systems, Vol. PAS-98, No. 6, pp. 2047-2054, November/December 1979.

[139] Reliability Test Task Force, “The IEEE Reliability Test System-1996,” IEEE Transactions on Power Systems, Vol.14, No. 3, August 1999.

[140] F. Yang, A. P. Meliopoulos, G. J. Cokkinides, and G. K. Stefopoulos, "Contingency Simulation Using Single Phase Quadratized Power Flow," $\underline{9}^{\text {th }}$ International Conference on Probabilistic Methods Applied to Power Systems, June 2006. 
[141] F. Yang, A. P. S. Meliopoulos, G. J. Cokkinides, and G. Stefopoulos, "A Bulk Power System Reliability Assessment Methodology," accepted by European Transactions on Electric Power, 2007.

[142] F. Yang, A. P. S. Meliopoulos, G. J. Cokkinides, and G. Stefopoulos, "A Comprehensive Approach for Bulk Power System Reliability Assessment," accepted by Power Tech Conference, June 2007. 